LIGIA MARIA MOREIRA ZORELLO

Dynamic CPU frequency scaling using machine learning for NFV applications 


\title{
Dynamic CPU frequency scaling using machine learning for NFV applications
}

\author{
Dissertação apresentada à Escola Politéc- \\ nica da Universidade de São Paulo para \\ obtenção do Título de Mestre em Ciências.
}




\section{Dynamic CPU frequency scaling using machine learning for NFV applications}

Dissertação apresentada à Escola Politécnica da Universidade de São Paulo para obtenção do Título de Mestre em Ciências.

Área de concentração:

Engenharia da Computação

Orientador:

Profa. Dra. Tereza Cristina Melo de Brito Carvalho

Co-orientador:

Dr. Catalin Meirosu 
Autorizo a reprodução e divulgação total ou parcial deste trabalho, por qualquer meio convencional ou eletrônico, para fins de estudo e pesquisa, desde que citada a fonte.

Este exemplar foi revisado e corrigido em relação à versão original, sob responsabilidade única do autor e com a anuência de seu orientador.

São Paulo, de de

Assinatura do autor:

Assinatura do orientador:

\section{Catalogação-na-publicação}

Zorello, Ligia Maria Moreira

Dynamic CPU frequency scaling using machine learning for NFV applications / L. M. M. Zorello -- versão corr. -- São Paulo, 2018.

$95 \mathrm{p}$.

Dissertação (Mestrado) - Escola Politécnica da Universidade de São Paulo. Departamento de Engenharia de Computação e Sistemas Digitais.

1.Eficiência Energética 2.Aprendizado de Máquina 3.Redes de Computadores I.Universidade de São Paulo. Escola Politécnica.

Departamento de Engenharia de Computação e Sistemas Digitais II.t. 


\section{ACKNOWLEDGMENTS}

I would like to express my gratitude to my advisor, Prof. Dr. Tereza Cristina Melo de Brito Carvalho, for the opportunity to pursue a Master Degree and for her support during the development of this work. I would also like to thank my co-advisor, Dr. Catalin Meirosu, for his guidance and all his valuable tips and technical contributions to the development of my master thesis.

I would also like to express my warmest thanks to my parents, Gilberto Zorello e Suzana Andrade Moreira Zorello, to my sister, Raquel Maria Moreira Zorello, and to boyfriend, Davide Felice Redaelli, for their affection and support at all times. Further, to all my family and friends for their continuous encouragement and understanding.

Thanks to everyone with whom I had the chance to work at LASSU-PCS-EPUSP (Laboratory of Sustainability of the Department of Computer Engineering and Digital Systems of Polytechnique School of Universidade de São Paulo) for the technical support and for the shared experiences during the development of this work.

I would like to thank CNPq (Conselho Nacional de Desenvolvimento Científico e Tecnológico), FDTE (Fundação para o Desenvolvimento Tecnológico da Engenharia) and to Ericsson Brazil for the financial support. 
"We keep moving forward, opening up new doors and doing new things because we're curious... and curiosity keeps leading us down new paths."

-Walt Disney 


\section{RESUMO}

O crescimento do setor de Tecnologia da Informação e Comunicação está aumentando a necessidade de melhorar a qualidade de serviço e a eficiência energética, pois o setor já ultrapassou a marca de 12\% do consumo energético global em 2017. Data centers correspondem a grande parte desse consumo, representando cerca de $15 \%$ dos gastos com energia do setor Tecnologia Informação e Comunicação; além disso, o subsistema que gera mais custos para operadores de data centers é o de servidores e armazenamento. Muitas soluções foram propostas a fim de reduzir o consumo de energia com servidores, como o uso de escalonamento dinâmico de tensão e frequência, uma tecnologia que permite adaptar o consumo de energia à carga de trabalho, embora atualmente não sejam otimizadas para o processamento do tráfego de rede. Nessa dissertação, foi desenvolvido um método de controle usando um mecanismo de previsão baseado na análise do tráfego que chega aos servidores. Os algoritmos de aprendizado de máquina baseados em Redes Neurais e em Máquinas de Vetores de Suporte foram utilizados, e foi verificado que é possível reduzir o consumo de energia em até $12 \%$ em servidores com processador Intel Sandy Bridge e em até $21 \%$ em servidores com processador Intel Haswell quando comparado com a frequência máxima, que é atualmente a solução mais utilizada na indústria.

Palavras-Chave - Virtualização de funções de rede. Aprendizado de máquina. Eficiência energética. Escalonamento dinâmico de tensão e frequência 


\section{ABSTRACT}

Growth in the Information and Communication Technology sector is increasing the need to improve the quality of service and energy efficiency, as this industry has already surpassed $12 \%$ of global energy consumption in 2017. Data centers correspond to a large part of this consumption, accounting for about $15 \%$ of energy expenditure on the Information and Communication Technology domain; moreover, the subsystem that generates the most costs for data center operators is that of servers and storage. Many solutions have been proposed to reduce server consumption, such as the use of dynamic voltage and frequency scaling, a technology that enables the adaptation of energy consumption to the workload by modifying the operating voltage and frequency, although they are not optimized for network traffic. In this thesis, a control method was developed using a prediction engine based on the analysis of the ongoing traffic. Machine learning algorithms based on Neural Networks and Support Vector Machines have been used, and it was verified that it is possible to reduce power consumption by up to $12 \%$ on servers with Intel Sandy Bridge processor and up to $21 \%$ in servers with Intel Haswell processor when compared to the maximum frequency, which is currently the most used solution in the industry.

Keywords - Network Function Virtualization. Machine learning. Energy efficiency. Dynamic Voltage and Frequency Scaling 


\section{LIST OF FIGURES}

Figure 1 Global Mobile data traffic (ExaBytes per month). . . . . . . . . . . 1

Figure 22012 Energy consumption of countries compared to ICT sector. . . 2

Figure 3 Energy consumed by each subsystem in respect to the entire DC. . 3

Figure 4 Thesis Organization. . . . . . . . . . . . . . . . 6

Figure 5 CMOS area scaling trend. . . . . . . . . . . . . . 8

Figure 6 Power domains supported by RAPL. . . . . . . . . . . . . . . . . . 12

$\begin{array}{lll}\text { Figure } 7 & \text { Techniques to reduce energy consumption in data centers servers. } & 17\end{array}$

Figure 8 Activities followed to propose a new system architecture. . . . . . . 24

Figure 9 Testing environment implementation. . . . . . . . . . . . . . . 26

Figure 10 Energy consumption per transmission rate for five frequencies. . . . 27

Figure 11 Average energy consumption per frequency when the transmission rate is 9,000 pps. . . . . . . . . . . . . . . . . . . . . . . . 28

Figure 12 DVFS Prediction Engine Architecture. . . . . . . . . . . . . . 30

Figure 13 Neural Network structure for prediction. . . . . . . . . . . . . . 33

Figure 14 Training flow chart. . . . . . . . . . . . . . . . 36

Figure 15 Training sequence diagram. . . . . . . . . . . . . . 37

Figure 16 Real-time flow chart. . . . . . . . . . . . . . . 38

Figure 17 Real-time sequence diagram. . . . . . . . . . . . . . . . . 39

Figure 18 Testing environment implementation. . . . . . . . . . . . . . . 41

Figure 19 Energy consumption per number of packets received for fifteen fre-

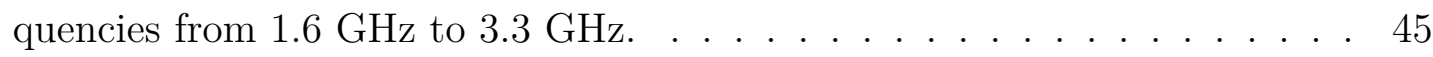

Figure 20 Number of cycles executed per number of packets received for fifteen frequencies from $1.6 \mathrm{GHz}$ to $3.3 \mathrm{GHz} \ldots \ldots \ldots$. . . . . . . . . 45

Figure 21 Energy per number of packets received for eight frequencies from $1.6 \mathrm{GHz}$ to $3.3 \mathrm{GHz} . \ldots \ldots \ldots$. . . . . . . . . . . . . . . 46 
Figure 22 Cycles executed per number of packets received for eight frequencies from $1.6 \mathrm{GHz}$ to $3.3 \mathrm{GHz} . \ldots \ldots$. . . . . . . . . . . . . . . 46

Figure 23 Energy consumption per transmission rate with 20 and 200 neurons. 47

Figure 24 Energy consumption in time for DVFS Prediction Engine, maximum frequency and ondemand governor at 10,000 pps. . . . . . . . . . . . . . . 49

Figure 25 Energy efficiency per transmission rate for DVFS Prediction Engine, maximum frequency and ondemand governor at 10,000 pps. . . . . . . . . 49

Figure 26 Energy consumption in time for DVFS Prediction Engine, maximum frequency and ondemand governor at 15,000 pps. . . . . . . . . . . . . . 50

Figure 27 Energy efficiency per transmission rate for DVFS Prediction Engine, maximum frequency and ondemand governor at 15,000 pps. . . . . . . . . 50

Figure 28 Energy consumption per transmission rate for DVFS Prediction Engine with 20 and 200 neurons, maximum frequency, and ondemand governor. 51

Figure 29 Energy consumption per number of packets received for thirteen frequencies from $1.2 \mathrm{GHz}$ to $2.4 \mathrm{GHz}$. . . . . . . . . . . . . . . . . . 54

Figure 30 Number of cycles executed per number of packets received for thirteen frequencies from $1.2 \mathrm{GHz}$ to $2.4 \mathrm{GHz}$. . . . . . . . . . . . . . . . . . 54

Figure 31 Energy consumption per number of packets received for seven frequencies from $1.2 \mathrm{GHz}$ to $2.4 \mathrm{GHz}$. . . . . . . . . . . . . . . . . . 55

Figure 32 Number of cycles executed per number of packets received for seven frequencies from $1.2 \mathrm{GHz}$ to $2.4 \mathrm{GHz}$. . . . . . . . . . . . . . . . . . 55

Figure 33 Energy consumption in time for DVFS Prediction Engine with ANN and SVM, maximum frequency and ondemand governor at 15,000 pps. . . 58

Figure 34 Energy efficiency per transmission rate for DVFS Prediction Engine with ANN and SVM, maximum frequency and ondemand governor at 15,000 pps. . . . . . . . . . . . . . . . . . . . 58

Figure 35 Energy consumption in time for DVFS Prediction Engine with ANN and SVM, maximum frequency and ondemand governor at 33,000 pps. . . 59

Figure 36 Energy efficiency per transmission rate for DVFS Prediction Engine with ANN and SVM, maximum frequency and ondemand governor at $33,000 \mathrm{pps}$ 
Figure 37 Energy consumption per transmission rate for DVFS Prediction Engine with ANN and SVM, maximum frequency and ondemand governor. 61 


\section{LIST OF TABLES}

Table 1 Energy efficiency methods for NFV applications. . . . . . . . . . . 23

Table 2 List of traffic characterization features. . . . . . . . . . . . 31

Table 3 Transmission rate selected per frequency for training with local server. 43

Table 4 Highest accuracy values obtained for each set of ANN parameters in local server. . . . . . . . . . . . . . . . . . . . . 44

Table 5 Transmission rate selected per frequency for training with CloudLab server. . . . . . . . . . . . . . . . . 53

Table 6 Highest accuracy values obtained for each set of ANN parameters in CloudLab server. . . . . . . . . . . . . . . . 56

Table 7 Highest accuracy values obtained for each set of SVM parameters for CloudLab server. . . . . . . . . . . . . . . . 56

Table 8 Requirements fulfillment . . . . . . . . . . . . 62

Table $9 \quad$ Accuracy obtained for each set of ANN parameters in local server. . 75

Table 10 Accuracy obtained for each set of ANN parameters in CloudLab server. 77

Table 11 Accuracy obtained for each set of SVM parameters for CloudLab server. . . . . . . . . . . . . . . . . . 79 


\section{TERMINOLOGY}

ACPI

ANN

API

CGP

CMOS

$\mathrm{CNPq}$

CPU

DC

DPDK

DPM

DRAM

DVFS

E2C

eDRAM

EPUSP

FDTE

FIVR

FR

H-EARtH

ICT

IDS

KVM
Advanced Configuration and Power Interface

Artificial Neural Network

Application Programming Interface

Contacted Gate Pitch

Complementary Metal-Oxide-Semiconductor

Conselho Nacional de Desenvolvimento Científico e Tecnológico

Central Processing Unit

Data Center

Data Plane Development Kit

Dynamic Power Management

Dynamic Random Access Memory

Dynamic Voltage and Frequency Scaling

Energy Efficiency to Clouds

Embedded Dynamic Random Access Memory

Polytechnic School of USP

(Escola Politécnica da USP)

Fundação para o Desenvolvimento Tecnológico da Engenharia

Fully Integrated Voltage Regulator

Functional Requirement

Heterogeneous Energy-Aware Race to Halt

Information and Communication Technology

Intrusion Detection System

Kernel-based Virtual Machine 


\begin{tabular}{|c|c|}
\hline LASSU-PCS-EPUSP & $\begin{array}{l}\text { Laboratory of Sustainability of the PCS-EPUSP } \\
\text { (Laboratório de Sustentabilidade PCS-EPUSP) }\end{array}$ \\
\hline MLP & Multi-Layer Perceptron \\
\hline MSR & Model-Specific Register \\
\hline NIC & Network Interface Card \\
\hline NFV & Network Function Virtualization \\
\hline OS & Operating System \\
\hline OSI & Open System Interconnection \\
\hline $\mathrm{PCU}$ & Power Control Unit \\
\hline PCS & $\begin{array}{l}\text { Department of Computer Engineering and Digial Systems } \\
\text { (Departamento de Engenharia da Computação e Sistemas Digitais) }\end{array}$ \\
\hline $\mathrm{PCH}$ & Platform Controller Hub \\
\hline pps & Packets Per Second \\
\hline QoS & Quality of Service \\
\hline RAPL & Running Average Power Limit \\
\hline SRAM & Static Random Access Memory \\
\hline SVM & Support Vector Machines \\
\hline TCP & Transmission Control Protocol \\
\hline UDP & User Datagram Protocol \\
\hline USP & Universidade de Sao Paulo \\
\hline VM & Virtual Machine \\
\hline VNF & Virtualized Network Function \\
\hline vNIC & Virtual Network Interface Card \\
\hline
\end{tabular}




\section{CONTENTS}

1 Introduction 1

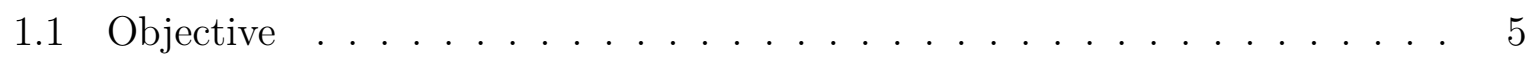

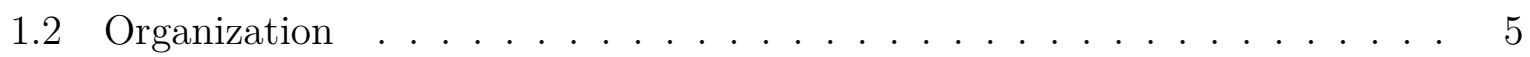

$\begin{array}{lll}2 & \text { Energy efficiency background } & 7\end{array}$

2.1 Processors frequency $\ldots \ldots \ldots \ldots \ldots \ldots$

2.2 Dynamic Voltage and Frequency Scaling . . . . . . . . . . . . . . . 9

2.3 Running Average Power Limit . . . . . . . . . . . . . . . . . . 11

2.4 Evolution on Intel processors . . . . . . . . . . . . . . . . . . 13

2.5 Chapter final remarks . . . . . . . . . . . . . . . . . . . . 13

$\begin{array}{lll}3 & \text { Energy efficiency approaches } & 15\end{array}$

3.1 Energy efficiency challenges for Network Function Virtualization . . . . . . 15

3.2 Reducing energy consumption in data center servers with NFV applications . . . . . . . . . . . . . . 16

3.2.1 Server consolidation techniques . . . . . . . . . . . . . 17

3.2.2 Server throttling techniques . . . . . . . . . . . . . 19

3.3 Chapter final remarks . . . . . . . . . . . . . . . . . 21

4 DVFS prediction engine $\quad 24$

4.1 Energy saving potential . . . . . . . . . . . . . . . 25

4.2 Requirements Specification . . . . . . . . . . . . . . . . 28

4.3 System architecture . . . . . . . . . . . . . . . . . . 29

4.3 .1 User level modules . . . . . . . . . . . . . . . . . . 31

4.3.1.1 Artificial Neural Networks . . . . . . . . . . . . . 32 
4.3.1.2 Support Vector Machines . . . . . . . . . . . . . . . 33

4.3 .2 Kernel level modules . . . . . . . . . . . . . . . . . . . . . . . 34

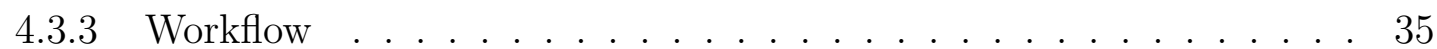

4.3.3.1 Training phase workflow . . . . . . . . . . . 35

4.3.3.2 Real-time phase workflow . . . . . . . . . . . . 35

4.4 Chapter final remarks . . . . . . . . . . . . . . . . . . . . . . . 37

5 Implementation and Results Description 40

5.1 Testing environment . . . . . . . . . . . . . . . . . 40

5.2 Local server . . . . . . . . . . . . . . . . . . . . . . . . 42

5.2 .1 Training results . . . . . . . . . . . . . . . . 43

5.2 .2 Real-time results . . . . . . . . . . . . . . . . . 44

5.3 CloudLab server . . . . . . . . . . . . . . . . . . . 52

5.3 .1 Training results . . . . . . . . . . . . . . . . 52

5.3 .2 Real-time results . . . . . . . . . . . . . 56

5.4 Requirement Fulfillment . . . . . . . . . . . . . . . . . . 61

5.5 Chapter Final Remarks . . . . . . . . . . . . . . . . . . . . . 63

6 Final Considerations $\quad 65$

6.1 Main Contributions . . . . . . . . . . . . . . . . . 65

6.2 Publications . . . . . . . . . . . . . . . . . 67

6.3 Future work . . . . . . . . . . . . . . . . . . 67

$\begin{array}{lr}\text { References } & 69\end{array}$

$\begin{array}{ll}\text { Appendix A - Accuracy for local server } & 75\end{array}$

$\begin{array}{ll}\text { Appendix B - Accuracy for CloudLab server } & 77\end{array}$ 


\section{INTRODUCTION}

The increase on the number of mobile devices is leading to a growth on the use of new technologies and services, such as social media, gaming, cloud computing and video streaming; consequently, a great amount of information is exchanged in the network. According to Ericsson (2017), the number of mobile subscriptions should surpass 9 billion by 2023 , which represents a growth of more than $15 \%$ when compared to 2017 . Also, a sustained increase in traffic is predicted until 2023, to about 110 Exabytes per month and more than eight times the traffic of 2017 as observed in Figure 1.

Figure 1: Global Mobile data traffic (ExaBytes per month).

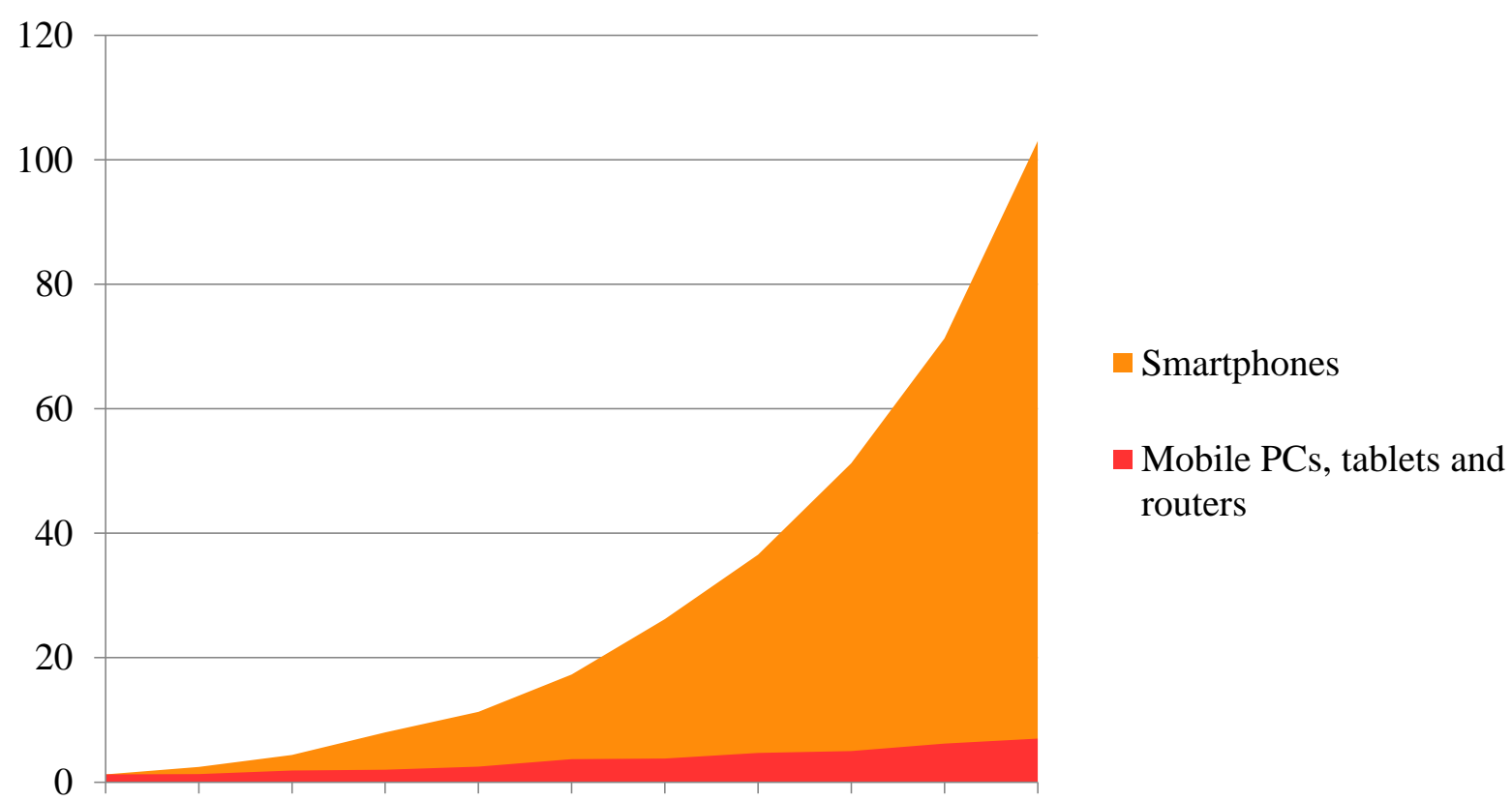

20132014201520162017201820192020202120222023

Source: (ERICSSON, 2017)

The boost of mobile devices and data traffic drives a rise in the energy consumption of Information and Communication Technology (ICT) sector. Indeed, Cook (2012) shows that ICT is one of the sectors that is growing the most and the fastest, both economically and in terms of energy consumption. In 2012, it was estimated that the ICT sector 
consumed more than $7 \%$ of global generated energy. In addition, projections indicate that it could have surpassed $12 \%$ in 2017 and will continue growing at an annual rate of $7 \%$ until 2030 (COOK et al., 2017). Figure 2 presents the energy consumption in 2012 of the most power demanding countries compared to the ICT sector. From this graph, it is clear that, if ICT were considered a country, it would be among the most energy consuming ones.

Figure 2: 2012 Energy consumption of countries compared to ICT sector.

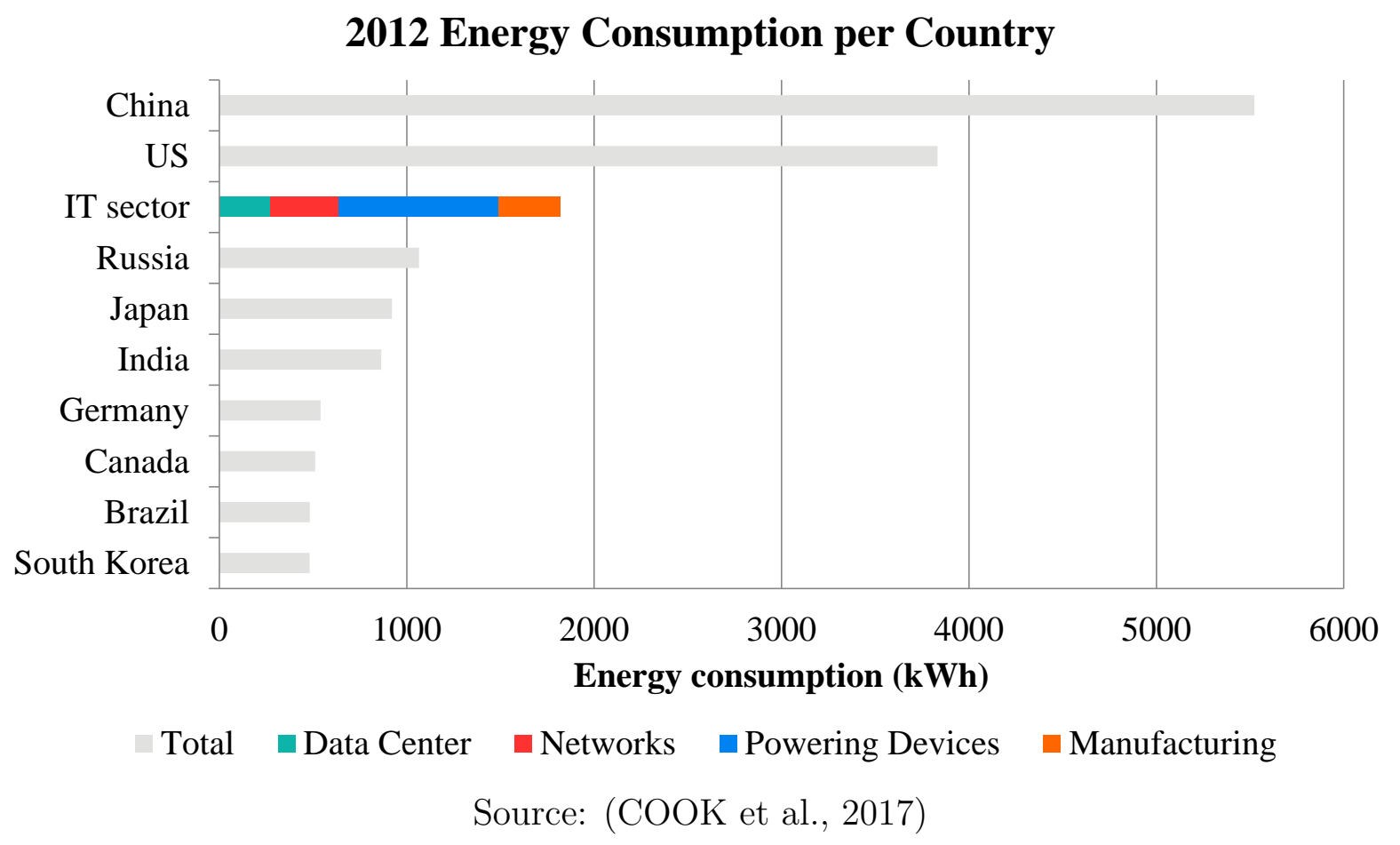

Considering only data centers and cloud computing servers, the power demand was approximately $15 \%$ of the ICT total energy consumption in 2012, representing $2 \%$ of global electricity demand and is increasing drastically (COOK, 2012; COOK et al., 2017). Indeed, some reports estimate that this value should rise to more than $8 \%$ by 2020 (KRZYWDA et al., 2018). According to Pettey (2016), costs related to powering Data Center (DCs) are increasing about 10\% per year, not only because of the higher costs in kilowatthour (kWh), but also due to the servers high energy consumption, which is expected to reach $13 \%$ by 2030 .

Pelley et al. (2009) divide the DCs energy consumption in five subsystems, namely servers and storage, power conditioning, cooling, networking and lighting. Figure 3 presents the typical power distribution in 2009.

These values may vary according to the DC location and to the operations performed 
Figure 3: Energy consumed by each subsystem in respect to the entire DC.

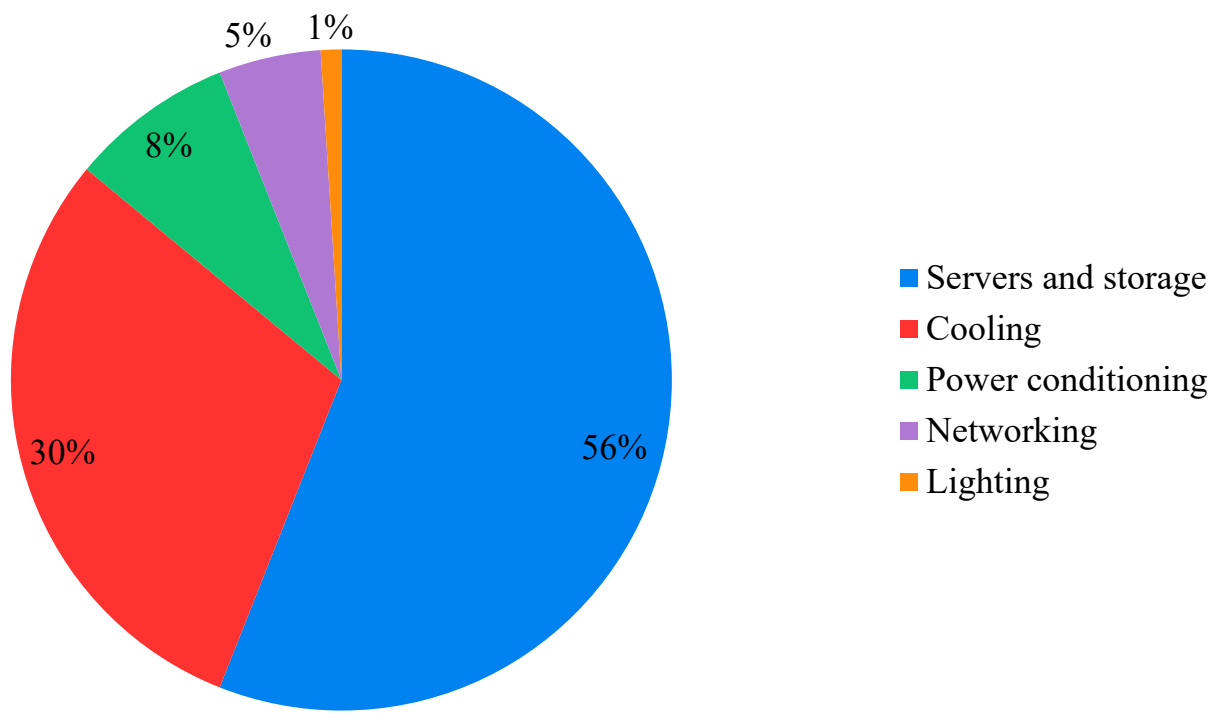

Source: (PELLEY et al., 2009)

in it. Nevertheless, this result shows that among all DC subsystems, the servers and storage is the most energy consuming, representing $56 \%$ of the total. Since total power demand in DCs has increased greatly in the past years and is forecast to continue growing, it is also expected that servers will also consume more and more energy. Therefore, numerous studies to find solutions that improve server energy efficiency have been developed.

Some of the solutions that have been brought up are based on the fact that the component that consumes more energy in servers is the Central Processing Unit (CPU), and recent high-end server-grade CPUs may have up to 28 cores and a data sheet nominal power consumption of over 400 Watts (INTEL, 2018b). The Dynamic Voltage and Frequency Scaling (DVFS) capability of the CPU is a tool that enables modifying both frequency and voltage in order to reduce power consumption, while meeting performance requirements, and it is widely used in enterprise environments (KUANG; BHUYAN; KLEFSTA, 
2012). Nevertheless, it may lead to performance degradation in some applications; the improvement of performance has therefore been widely studied in different application domains.

However, even if techniques such as DVFS prevail in regular DCs and desktops, the use of DVFS in Network Function Virtualization (NFV) applications has not yet been widely explored, because the built-in DVFS mechanisms can cause packet latency (PAN et al., 2018). NFV is a technology that is under development to ensure Quality of Service (QoS) in packet processing while enabling services scalability (MIJUMBI et al., 2016). It drives the evolution of telecommunication provider infrastructure towards virtualizing physical devices as much as possible. To this end, it replaces physical appliances deployed in the field with software running on commodity servers, which are known as Virtual Network Functions (VNFs) (XU et al., 2016). To achieve these benefits of NFV, telecommunication networks require very high constraints in terms of performance. Therefore, most of the research in this domain approaches the packet processing performance improvement (XU et al., 2016).

Because NFV requires efficient processing, setting compute servers continuously to high-performance operating modes is a typical NFV approach for achieving predictable operations. Moreover, it is common practice to overprovision communication and computing resources in order to cope with the not-constant workload in servers due to traffic fluctuation (KLIAZOVICH; BOUVRY; KHAN, 2012). Hence, energy savings are completely neglected as most operators tend to disable DVFS techniques (PAN et al., 2018), generating significant expenses for the operator. It is thus necessary to provide solutions in order to reduce the energy consumption of DC servers that feature NFV applications.

In addition, the CPU momentary usage - and, consequently, the energy consumption - differs for each packet received or forwarded by the VNF, also depending on its content and on the processing code executed by the application. The energy consumption also varies according to different processor instructions, depending not only on the instructions themselves, but also on where the data needed for the execution resides. Thus, the variation in the network traffic induces a non-linear relation between computing resources consumed and the traffic processed for many NFV applications (KUANG; BHUYAN; KLEFSTA, 2012; XU et al., 2016). This can therefore be treated as a classification problem, in which different traffic profiles are classified into performance mode categories.

Along with the differences between the processors architectures and the great number of parameters that can be used to classify the traffic data into various energy performance 
modes, each NFV application would require a different analytical solution to determine the optimal operation mode. This approach is not feasible from a practical implementation perspective, since it would require significant amount of manual work to develop a solution for each application, which could lead to the introduction of errors in the process. Determining the optimum energy performance mode to any NFV application is therefore a problem that would be extremely difficult to approach analytically.

Based on the need for classifying the traffic into different energy performance modes and to automatically tune the solution to any NFV application, machine learning techniques could be incorporated in the system. This method is considered to be promising in approaching similar problems to the one discussed in this thesis, because of its ability to adapt itself to new environments (ISLAM et al., 2018).

\subsection{Objective}

In this thesis, it is intended to achieve the Hypothesis 1:

Hypothesis 1. Dynamic scaling the CPU operating frequency with the use of machine learning enables the reduction in the energy consumption in data centers with NFV applications.

In order to achieve this goal, a prediction engine that analyzes the ongoing traffic was developed. It captures the network traffic features and selects the best frequency, i.e. the best frequency that reduces the energy consumption while maintaining sufficient throughput for packet processing performance.

\subsection{Organization}

Figure 4 presents the thesis organization.

Chapter 2 reviews the background on energy efficiency potential in the ICT sector, explaining the DVFS technique, Running Average Power Limit (RAPL) interface, and the hardware limitations. Chapter 3 presents the existing solutions for energy efficiency in data centers servers with NFV applications, including a brief description of NFV challenges in terms of reducing energy consumption. Chapter 4 describes the proposed architecture for the DVFS Prediction Engine, providing details on the development of certain capabilities. Chapter 5 presents the testbed implementation and reports the results for 
Figure 4: Thesis Organization.

\begin{tabular}{|c|c|c|c|c|}
\hline $\begin{array}{l}\text { Liter } \\
\text { Review an }\end{array}$ & $\begin{array}{l}\text { ature } \\
\text { d Analysis }\end{array}$ & $\begin{array}{l}\text { DVFS Prediction Engine } \\
\text { Design and Development }\end{array}$ & $\begin{array}{c}\text { Results } \\
\text { Evaluation }\end{array}$ & $\begin{array}{c}\text { Final } \\
\text { Considerations }\end{array}$ \\
\hline Chapter 2 & Chapter 3 & Chapter 4 & Chapter 5 & Chapter 6 \\
\hline
\end{tabular}

Source: Author

the experimental validation, which were divided in two parts: the results from the server with Sandy Bridge microarchitecture and from the one with Haswell microarchitecture. To conclude, Chapter 6 presents the final remarks, the publications associated to this thesis and future work. 


\section{ENERGY EFFICIENCY BACKGROUND}

This chapter discusses the background regarding energy consumption and efficiency in servers. First, Section 2.1 presents the evolution of processors energy consumption, explaining the main factors that contribute to the increase of energy demand. Next, Section 2.2 characterizes the Dynamic Voltage and Frequency Scaling (DVFS) and its implementation in current processors. Section 2.3 describes the Running Average Power Limit (RAPL), which can be used as means for performing energy measurements, and the limitations associated to it. After that, Section 2.4 presents the evolutions of Intel processors when considering the usage of DVFS techniques and RAPL interface. Finally, Section 2.5 concludes this chapter.

\subsection{Processors frequency}

The most important component of processors is the set of transistors, which are semiconductor devices that act as switches controlled by a voltage. The interconnection of multiple transistors enables the construction of Complementary Metal-Oxide-Semiconductor (CMOS) integrated circuits and, consequently, logical gates - such as inverters, AND and OR gates, multiplexers. They are then combined into different units in order to implement a processor capable of arithmetic and other complex operations (DESCHAMPS; VALDERRAMA; TERES, 2017).

Increasing the transistors density requires a sustained effort from researchers and developing areas to ensure improvements in the overall processors performance. In 1965, Moore (2006, p. 34) forecast that the number of components in a CMOS integrated circuit would double every year:

"The complexity for minimum component costs has increased at a rate of roughly a factor of two per year. Certainly, over the short term, this rate can be expected to continue, if not to increase. Over the longer term, the rate of increase is a bit more uncertain, although there is no reason to believe it 
will not remain nearly constant for at least 10 years. That means by 1975 , the number of components per integrated circuit for minimum cost will be $65,000 . "$

Figure 5 presents the density progress considering Static Random Access Memory (SRAM) in blue dots, wire-to-wire spacing (M1 pitch) in green triangles, and transistors spacing (Contacted Gate Pitch - CGP) in red squares since 1994. Although the progress in density is slowing, it is still rapid, being, therefore, necessary to increase the voltage in order to operate the CMOS integrated circuit.

Figure 5: CMOS area scaling trend.

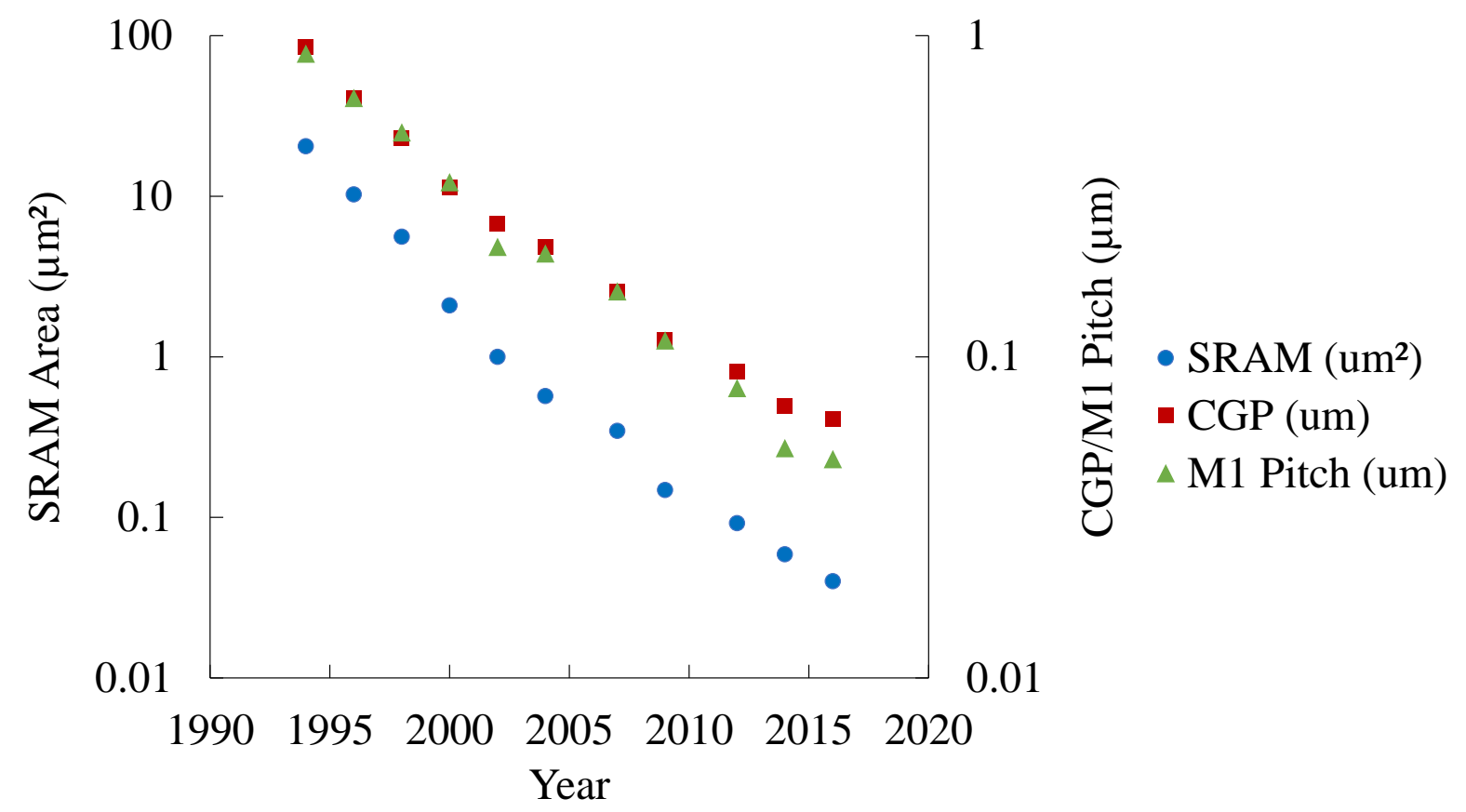

Source: (THEIS; WONG, 2017)

In addition, the speed in which these operations are executed is limited by a clock frequency, i.e. the frequency in which the transistor is able to switch between on and off correctly; thus, the overall processor performance depends on this value. Clock frequency increase has been investigated in the past years, which enabled achieving performance scaling. Indeed, Borkar et al. (2005) predicted that the gains would have increased by 80\% when comparing the architectures in 2015 and in 2005.

The clock frequency in most CMOS integrated circuits is dependent on the supply voltage and can be calculated as (SAHA; RAVINDRAN, 2012): 


$$
f=k \times \frac{\left(V_{d d}-V t\right)^{2}}{V_{d d}}
$$

where $k$ is a constant, $V_{d d}$ is the supply voltage, and $V_{t}$ is a threshold voltage. Together with the growth in transistors density inside processors integrated circuits, the frequency scaling leads to greater power consumption. Additionally, the power consumption of a CMOS integrated circuit can be calculated as follows (SULEIMAN; IBRAHIM; HAMARASH, 2005):

$$
P_{C M O S}=P_{\text {dynamic }}+P_{\text {static }}+P_{\text {shortcircuit }}
$$

where $P_{\text {dynamic }}, P_{\text {static }}$, and $P_{\text {shortcircuit }}$ correspond to the dynamic, leakage, and shortcircuit power, respectively. The dynamic power is the most significant portion of the total CMOS power consumption and is expressed as (SULEIMAN; IBRAHIM; HAMARASH, 2005; SUEUR; HEISER, 2010):

$$
P_{\text {dynamic }} \propto C \times V^{2} \times f
$$

where $C, V$, and $f$ are the transistors capacitance, the supply voltage, and the operating clock frequency, respectively. Consequently, by reducing the voltage and the frequency, it is possible to reduce the total energy consumption in processors.

\subsection{Dynamic Voltage and Frequency Scaling}

Dynamic Voltage and Frequency Scaling (DVFS) is a generic name for techniques that adjust the voltage and operating frequency of a processor. It can be either chipwide, in which all cores are scaled at the same frequency, and per-core, in which each core can operate at different frequencies (KUANG; BHUYAN; KLEFSTA, 2012). The DVFS framework is well accepted as an approach to decrease the processors energy consumption, since it enables modifying the performance and energy capabilities by scaling the CPU voltage and frequency (KRZYWDA et al., 2018; SUEUR; HEISER, 2010). The CPU clock is coupled with a determined voltage level so that frequency scaling leads to simultaneous variation of the voltage values. Hence, from this point, references to CPU frequency also implies that the voltage is also implicitly being modified.

It is present in most processors architecture, such as AMD and Intel processors, and several authors proved it to be efficient when considering energy savings (KIM; BUYYA; 
KIM, 2007; RIZVANDI; TAHERI; ZOMAYA, 2011). Different processors manufacturers and generations have a variety of built-in DVFS mechanisms, in addition to the differences in power consumption that are inherent to the CMOS technology used. There are, however, some limitations associated to its use. Lower voltage and clock frequency naturally translate on reduced system performance; therefore, a trade-off between energy and processing efficiency needs to be taken into consideration when using this framework (LEE; ZOMAYA, 2011). Furthermore, it is coarse grained, presenting few possible settings, as a limited number of frequencies can be selected (KRZYWDA et al., 2018).

DVFS is supported at the operating system-level (SAHA; RAVINDRAN, 2012). In the Linux operating system, these capabilities are manageable through the cpufreq module, which specifies an interface to the frequency controlling policies in the CPU (PALLIPADI; STARIKOVSKIY, 2006). The software component that determines and defines the appropriate frequency according to the workload and a set of policies is known as a governor. Several types of governors are predefined in Linux. User-defined policies specify the governor and limit values for the parameters that could be set by the governors, i.e. minimum and maximum frequency that could be selected. Brodowski (2017) and Pallipadi \& Starikovskiy (2006) briefly describe the governors as:

- performance: sets frequency statically to the highest within the limits;

- powersave: sets frequency statically to the lowest within the limits;

- userspace: allows the user to set the frequency within the limits;

- ondemand: sets the frequency within the limits according to CPU workload;

- conservative: gradually sets the frequency within the limits according to the CPU workload.

These DVFS mechanisms only take into consideration the CPU workload to determine the frequency. In addition, the ondemand governor was developed as a mechanism to change the CPU clock dynamically inside the kernel, which would enable fast frequency scaling and present low overhead. It was therefore built as a simplistic algorithm that keeps the performance loss because of frequency reduction. To this end, it verifies periodically the CPU utilization, and, if it is over a certain threshold, the frequency is set to the maximum; else, it jumps to the lowest frequency and increases it successively until it reaches the right operating frequency for that workload (PALLIPADI; STARIKOVSKIY, 2006). 


\subsection{Running Average Power Limit}

At the processor level, the energy efficiency capabilities are exposed by the Running Average Power Limit (RAPL) interface on the chip (ZHANG; HOFFMAN, 2015). It was introduced in Intel Sandy Bridge micro-architecture and enables the power regulation to be executed directly in the hardware, receiving by the software an input containing the power limit information. Hence, the RAPL ensures that any changes to the power limit will be accurately respected and that the time to perform the necessary modifications is low. Moreover, the RAPL interface also allows measurement of energy consumption at a high sampling rates, around 1ms, with low overhead (KHAN et al., 2018).

RAPL values for determining the current energy consumption are obtained by reading the Model-Specific Register (MSR) interface (INTEL, 2018a). While the significance of the read values differs between Intel CPU generations (the estimate models of earlier generations were replaced by the actual measurements in newer ones), RAPL was found to be accurate in providing energy measurement values for the processor package, while the memory-related values were found to be less accurate (ZHANG; HOFFMAN, 2015; DESROCHERS; PARADIS; WEAVER, 2016).

Processors support up to five power domains, which are presented in Figure 6.

- Powerplane 0 domain: monitoring of all cores in the processor;

- Powerplane 1 domain: monitoring of the integrated graphics, hence, it is available only in desktop computers;

- DRAM domain: monitoring of the Dynamic Random Access Memory (DRAM);

- Psys domain: monitoring of the thermal and power specifications of the whole socket, including everything that is measured by the Package domain, the Platform Controller Hub (PCH) and the Embedded DRAM (eDRAM). This power domain is available only in some Skylake processors.

Based on this information, Powerplane 0 is the power domain that enables measuring the energy consumption of applications in the core. Nevertheless, some limitations are associated to this type of measurement. First, this power domain measures the entire socket, not allowing to determine the consumption of specific cores. Furthermore, it is not possible to measure the power by one of the running processes. 
Figure 6: Power domains supported by RAPL.
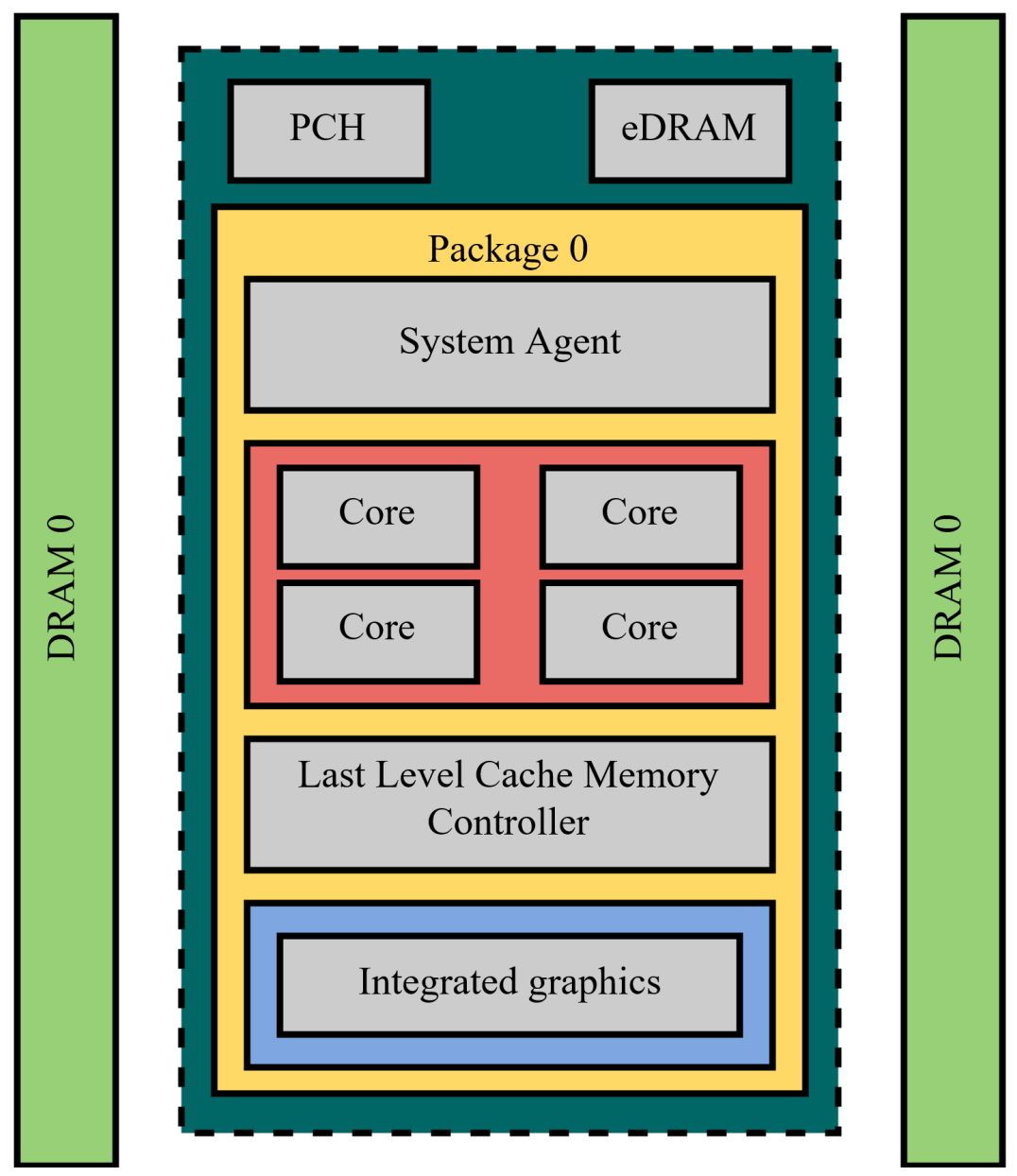

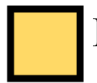

Package

$\square$ Powerplane 0

$\square$ Powerplane 1

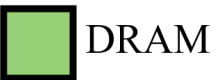

$\square$ Psys

Source: (KHAN et al., 2018)

To understand the energy consumption of software and processes in servers, Rieger \& Bockisch (2017) present a survey, in which they analyze the state of the art of multiple approaches for assessing software energy consumption. They verified that the existing tools are focused on specific platforms and can hardly be applied to custom codes. Moreover, most of the techniques analyzed only provide indications of consumption problems in the code, but not the real energy consumption related to programs or processes. Consequently, they concluded that there is not yet any accurate tool for measuring energy consumption per process. Furthermore, solutions such as RAPL provide data that enables application profiling, allowing to evaluate the energy consumption of the processes (GRANT et al., 2017). Methods using RAPL interface to estimate power usage can, therefore, be considered adequate for measuring applications. 


\subsection{Evolution on Intel processors}

Intel processors have increasingly advanced CPU energy efficiency capabilities for several generations. Rotem et al. (2012) present the Sandy Bridge (second generation Intel micro-architecture) design, which includes for the first time an energy-aware turbo algorithm that reduces energy consumption while delivering high performance. Rusu et al. (2015) describe the Ivytown architecture, a processor that uses the Ivy Bridge (third generation Intel micro-architecture). This architecture improves the Power Control Unit (PCU) to enable better power optimization.

Hackenberg et al. (2015) cover the details of Haswell processors (forth generation Intel micro-architecture), including energy efficient features that were included in this generation. The Fully Integrated Voltage Regulators (FIVRs) capability was first introduced in this micro-architecture, enabling fine-grained per-core voltage and frequency regulation. FIVRs is also available in Intel Xeon E5 v3 server processors (VARMA et al., 2015). In addition, Hackenberg et al. (2015) executed a series of benchmarks showing that the introduction of this feature also improved the power measurements using RAPL, which became more accurate from the Haswell generation.

Doweck et al. (2017) describe the 6th-generation Intel microarchitecture, code-named Skylake. In terms of power management, the Skylake introduced the Intel Speed Shift technology, enabling the CPU to control the power, performance and energy efficiency instead of the Operational System (OS), which is then responsible for defining preferences such as minimum Quality of Service (QoS), maximum frequency and energy performance. In addition, an optimized energy-efficiency control was enabled by the Heterogeneous Energy-Aware Race to Halt (H-EARtH) algorithm (ROTEM et al., 2016), which selects the best core in which a determined application should be executed, achieving the optimal energy consumption by managing the core operating voltage and frequency.

\subsection{Chapter final remarks}

This chapter presents an overview about different techniques deployed for reducing power consumption and improving energy efficiency in server processors. This comprehension is necessary to evaluate existing solutions and to create a basis for proposing an architecture of a new system to reduce energy consumption.

One of the key aspects discussed in this chapter was the influence of voltage and fre- 
quency in the energy consumption and performance in servers. Based on this, techniques such as DVFS have been developed, in which the frequency and the voltage are adjusted according to the performance and energy policies in the processor. This solution can differ from generation to generation, as different generations may present distinct voltage and frequency values, and also DVFS capabilities. Moreover, features such as the number of cores and the cache capacity also influence the solutions.

Another key aspect considered was the use of RAPL interface to measure the applications energy consumption. This technique was proved to have an accurate estimation of the power demand. Some drawbacks are, however, associated to this type of computation, as it is not possible to calculate the energy consumption neither per-process nor per-core. The limitation of per-process measurement can be overcome by the possibility of surrounding the application execution with measurements calls, which enables estimation of processes power profiling. 


\section{ENERGY EFFICIENCY APPROACHES}

This chapter provides an overview of different approaches to reduce the energy consumption in Data Centers (DCs) servers with Network Function Virtualization (NFV) applications. Section 3.1 presents NFV basic concepts and some challenges related to the improvement of energy efficiency in this scenario. Next, Section 3.2 describes different categories of solutions to reduce energy consumption in DCs in general, also providing some techniques related to NFV applications. Finally, Section 3.3 concludes de chapter, presenting a table comparing different solutions described in this chapter.

\subsection{Energy efficiency challenges for Network Func- tion Virtualization}

As outlined in Chapter 1, the substantial increase in the demand for connectivity requires expansion and improvement of network infrastructure, which typically generates more costs to operators. NFV was therefore envisioned as a solution that enables more flexibility, scalability and lower costs to operators. The expenditure reduction comes from the virtualization of several network functions so that they can be located in standard servers instead of using proprietary hardware appliances (HAWILO et al., 2014). ETSI (2012, p. 5) defined NFV as:

"Network Functions Virtualisation (...) involves the implementation of network functions in software that can run on a range of industry standard server hardware, and that can be moved to, or instantiated in, various locations in the network as required, without the need for installation of new equipment."

Thanks to the use of virtualization paradigm, common network functions can be executed as software on commodity hardware and can easily move among different physical

platforms. These logical instances that are deployed in Virtual Machines (VMs) are known as Virtualized Network Functions (VNFs). Bolla et al. (2011) supported the fact 
that this technology can bring benefits in terms of energy consumption. Indeed, ETSI (2013) asserted that NFV brings the possibility of performing workload consolidation and location optimization, such as concentrating the VNF in fewer servers when the cores are light loaded during off-peak hours, for example. Hence, the quantity of physical network nodes could potentially drop because of the virtualization of network functions, which leads to a reduction in the DC total energy consumption (MIJUMBI et al., 2016). Nevertheless, NFV may consume a considerable amount of energy in large-scale networks due to requirements such as high-performance (XU et al., 2016). Therefore, studies and solutions to reduce energy consumption in DCs that run NFV applications have been proposed recently.

Xu et al. (2016) presented a study on NFV in which they evaluate the energy efficiency of different NFV implementations based on packets arriving in the network. Based on their measurements, they observed that smaller packets consume more power than larger ones, because they require higher CPU utilization as more headers must be processed.. Thus, different packet characteristics cause a diversity in processing cost and energy consumption. Furthermore, significant energy usage variation was measured for data plane implementations deploying different libraries, as well as for distinct software implementations of the same virtual network function. This work illustrates the complexity involved in characterizing NFV workloads.

In addition, NFV implementation brings important challenges such as performance trade-off. The approach of executing virtualized network functions on commodity hardware as much as possible naturally impacts the network performance that could be delivered by an individual function instance. Consequently, operators tend to disable energy efficiency techniques related to throttling server capabilities in order to keep the performance degradation the lowest possible (ETSI, 2012; PAN et al., 2018). Such strategy results in unnecessary energy consumption when the traffic does not require the full capacity of the node, while improvements such as reduction in packet transit delays are not significant with modern servers.

\subsection{Reducing energy consumption in data center servers with NFV applications}

Improving energy efficiency in DC servers has been substantially studied in the past years. Thus, several approaches that aim at enhancing resource manage to achieve better energy efficiency have been proposed in the literature. These approaches can be divided 
into two categories according to Krzywda et al. (2018), server consolidation and server throttling, as described in Figure 7. Section 3.2.1 and Section 3.2.2 explain briefly each category and provide solutions found in the literature that can be related to NFV applications.

Figure 7: Techniques to reduce energy consumption in data centers servers.

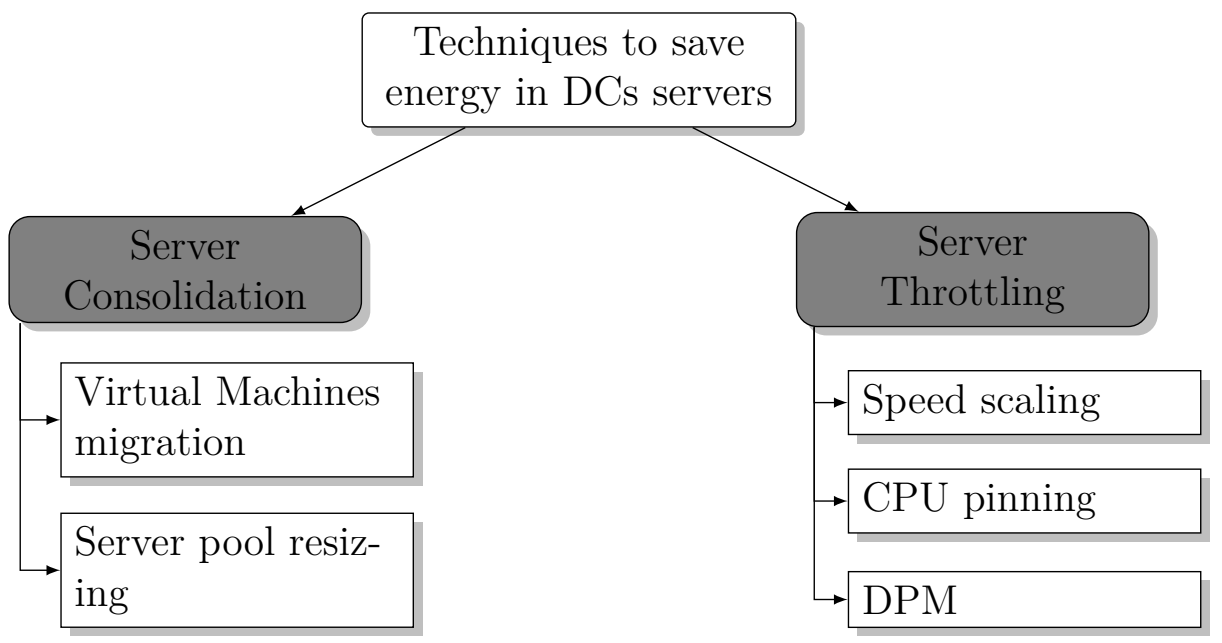

Source: Author

\subsubsection{Server consolidation techniques}

Server consolidation consists of reducing the amount of physical servers necessary to process the workload in a data center. According to Varasteh \& Goudarzi (2017), there are several techniques that could be used to provide server consolidation; they are all, however, based on two main features: migration of VMs and resizing the set of servers in the DC. Thus, the use of VMs in DCs enables increasing server utilization by migrating VMs from those that are underused and, consequently, turning them down (NEHRU et al., 2013).

In this scope, Varasteh \& Goudarzi (2017) presented a survey and a taxonomy on the different server consolidation techniques. They were divided into categories that take into account different features, such as the parameters considered when determining the server consolidations, the different goals of each technique, the algorithms used to solve the consolidation problem and the evaluation method. They verified that little work has been done in regard of network traffic evaluation, a parameter that could affect both the Quality of Service (QoS) and the processing performance in the DC. Hence, they concluded that many open research challenges still exist. In respect to NFV applications, they indicate that network-aware techniques could enable reducing energy consumption 
in DCs.

There are several optimization methods that aim at reducing energy consumption while guaranteeing the expected QoS in NFV infrastructures. Nevertheless, most solutions are based on models that assume the input as known in advance, which is often a wrong assumption, since it is not possible to know the CPU utilization nor the data throughput beforehand (MAROTTA; KASSLER, 2016).

As discussed in Section 3.1, the complexity to characterize NFV workloads makes it difficult to develop mechanisms that allocate servers and DCs resources to different virtualized network functions. Cao et al. (2017) treated this challenge as a classification problem and proposed the Elastic resource flexing for Network function VIrtualization (ENVI). This system enables resource-provisioning according to the workload dynamics. They use machine-learning models that were previously trained with NFV applications information and resource utilization to detect the need for scaling using ENVI. They tested the system in a real set of servers, by comparing them to other machine learning algorithms. Nevertheless, they do not evaluate the possibility of also reducing energy consumption with this technique.

Marotta \& Kassler (2016) developed a solution for energy-efficient robust NFV placement, optimizing both server and network energy consumption by applying workload consolidation techniques. They used a relatively simple model where the energy consumption of a VNF is determined by a quantity of generic resources requested by the NFV and the maximum variation of the resource utilization. Hence, they evaluated numerically the impact of the protection level determined by the operator, in which they define parameters that specify whether they are willing to take higher risks of losing QoS to save energy. The energy consumption models for resources were considered to vary linearly with the workload.

With the goal of reducing energy demand in DCs that execute NFV applications, Pan et al. (2018) proposed VNFMotion. It consists of a controller that aggregates VNF workloads in a lower quantity of cores whenever the CPU load is under a certain threshold. Because of the workload consolidation, VNFMotion controller puts the remaining idle cores in sleep mode and, consequently, it is possible to save energy. The controller also ensures that the DC will not present performance loss, since NFV applications require low latency in packet processing. This capability is enabled by an asynchronous CPU wakeup that sends a flag to VNFMotion whenever the CPU load is too high. 


\subsubsection{Server throttling techniques}

Server throttling consists of methods that improve energy efficiency of specific servers by dynamically adapting the resources capacities. Some of the techniques used in this approach are speed scaling, CPU pinning, and Dynamic Power Management (DPM), briefly explained as follows.

- Speed scaling: modifies the operating frequency and voltage of a processor using Dynamic Voltage and Frequency Scaling (DVFS) technique;

- $C P U$ pinning: defines which CPU cores to be used by each thread being executed in the processor;

- Dynamic Power Management: sets idle cores to sleep mode, i.e. powers them down almost completely.

Krzywda et al. (2018) evaluated the approaches of speed scaling and CPU pinning to understand the power consumption reduction and also the performance degradation. As expected, the average response time worsens when techniques to improve energy efficiency in servers are employed. They concluded that their ability to reduce energy consumption depends deeply on the server utilization, as they verified that high CPU load leads to greater potential amount of energy reduction. This research does not, however, evaluate dynamic algorithms nor utilize techniques that take into consideration more parameters to reduce energy consumption in servers. Moreover, Rossi et al. (2014) verified that when threads are fairly distributed through the cores of a CPU, DVFS could provide a significant reduction of energy consumption. However, while being able to save only about $10 \%$ energy, DVFS policies implemented in Linux increased response time of a web server by about $70 \%$.

Consequently, server-throttling approaches could potentially improve energy efficiency while maintaining sufficient processing performance; however, not only should they take into consideration the potential of reducing energy consumption based on the workload, but also they must analyze packet-processing performance.

Several solutions using server throttling technologies exist in the literature; however, only a few can actually be applied to NFV applications and networks energy efficiency, since solutions based on these techniques depend on the incoming traffic and on the hardware features in order to provide energy savings and sufficient network performance (BOLLA et al., 2011). 
Kuang, Bhuyan \& Klefsta (2012) proposed a system that seeks to optimize energy consumption by dynamically adjusting the per-core operating frequency and voltage based on the traffic rate for network applications. Given a certain packet inter-arrival rate, the system devises the optimal operation parameters for that rate, using DVFS and DPM techniques to reduce power consumption. They evaluated in a real server their solution by comparing its results in different NetBench network applications and verified a significant energy reduction in respect to a native system. However, using only the inter-arrival rate fails to account for the processing load associated with each packet, which can vary significantly depending on the size of the packets and the actual processing applied by the VNF instance.

Yu, Znati \& Yang (2015) developed a solution to be applied in a virtual router environment. It used the concept of DVFS to predict the frequency according to the evaluation of the network congestion, defined as the packet queue length and the link utilization. The optimal frequency to which the processor is scaled also ensures that the QoS requirements are met. They propose several algorithms, which vary in terms of the decision granularity and on the parameters used (queue length or link load). The solution was tested in a simulated environment in order to be compared to different solutions. They observed that, although the algorithms based on queue length provide more energy savings, all solutions are able to reduce considerably the energy consumption. While this approach indirectly accounts for the effects of the per-packet processing workloads, it requires careful dimensioning of the queue lengths while in operation such that packets are neither dropped unnecessarily nor delayed significantly more than needed.

Mochocki et al. (2007) presented a network-aware algorithm that aims at enhancing energy efficiency in terms of Network Interface Card (NIC) level. It uses the DVFS capabilities of the processor to coalesce packets together in order to optimize the utilization of the NIC. Therefore, it allows network-level techniques based on DPM to put the interface in sleep mode temporarily or reduce its transmission speed so that the NIC consumes less energy. The solution was evaluated both in a real server and via simulations. They verified that savings could reach $35 \%$ when their solution was applied in traffic-intensive scenarios. However, the NIC represents a very small part of the energy consumed by the server and is typically one order of magnitude less than the CPU.

Melo \& Petrucci (2018) introduced a DVFS-aware technique to improve energy efficiency in web browsing applications according to the network characteristics. They developed an intelligent energy manager, called Web Governor, which uses Support Vector Regression algorithm to determine the optimal operating frequency based on the network 
metrics, such as the packet delay and the web page content from the application layer. The experimental results showed that this solution enables significant energy savings with lower overhead than the performance governor and the interactive one, which was developed by Google for Android OS. This solution, developed in parallel with our proposal, is following a similar insight - one limitation of their approach is due to the fact that some features used for training the machine learning model are very specific to the web browsing application and cannot be easily generalized, which limits the possibility to generalize the solution.

\subsection{Chapter final remarks}

This chapter presents an overview of the current work on energy efficiency in DC servers with NFV applications. First, it introduces the challenges related to the implementation of NFV applications. As evidenced in (XU et al., 2016), VNFs exhibit a wide variation of CPU resource usage in relation to the bandwidth consumed, generating a complexity to characterize NFV workloads.

Solutions based on server consolidation and throttling are able to efficiently reduce energy consumption in DCs, enabling also the utilization in NFV applications. Server consolidation, in particular, is an approach that is intrinsic to the NFV concept, and several solutions to improve this technique have been proposed in the literature. Furthermore, server throttling techniques that depend on the traffic and hardware features were shown to provide energy savings, while meeting QoS requirements. The solutions studied in this chapter are summarized in Table 1.

Simple techniques that only employ inter-packet arrival times cannot cope with the aforementioned complexity. Techniques that are based on observing congestion indicators react only after the traffic was already impacted by a low power mode that was selected for the CPU. Workload consolidation solutions present good results in addressing diurnal variations in the traffic pattern, but exhibit too large overheads for adapting to fast timewise variations. A solution that could operate at the same tens of millisecond time scale as DVFS, and yet be able to adapt to the traffic and to the virtual network function that is executed in the cloud platform must be proposed.

As presented in the last row of Table 1, this thesis aims at proposing a solution in this regard. Therefore, the system merges machine learning algorithms to server throttling techniques to detect the best frequency according to parameters related to the packet 
information. Chapter 4 describes this system in detail. Moreover, it is important to evaluate the system in real servers, as simulations usually use simple models of energy consumption patterns, which may not reflect the reality. 


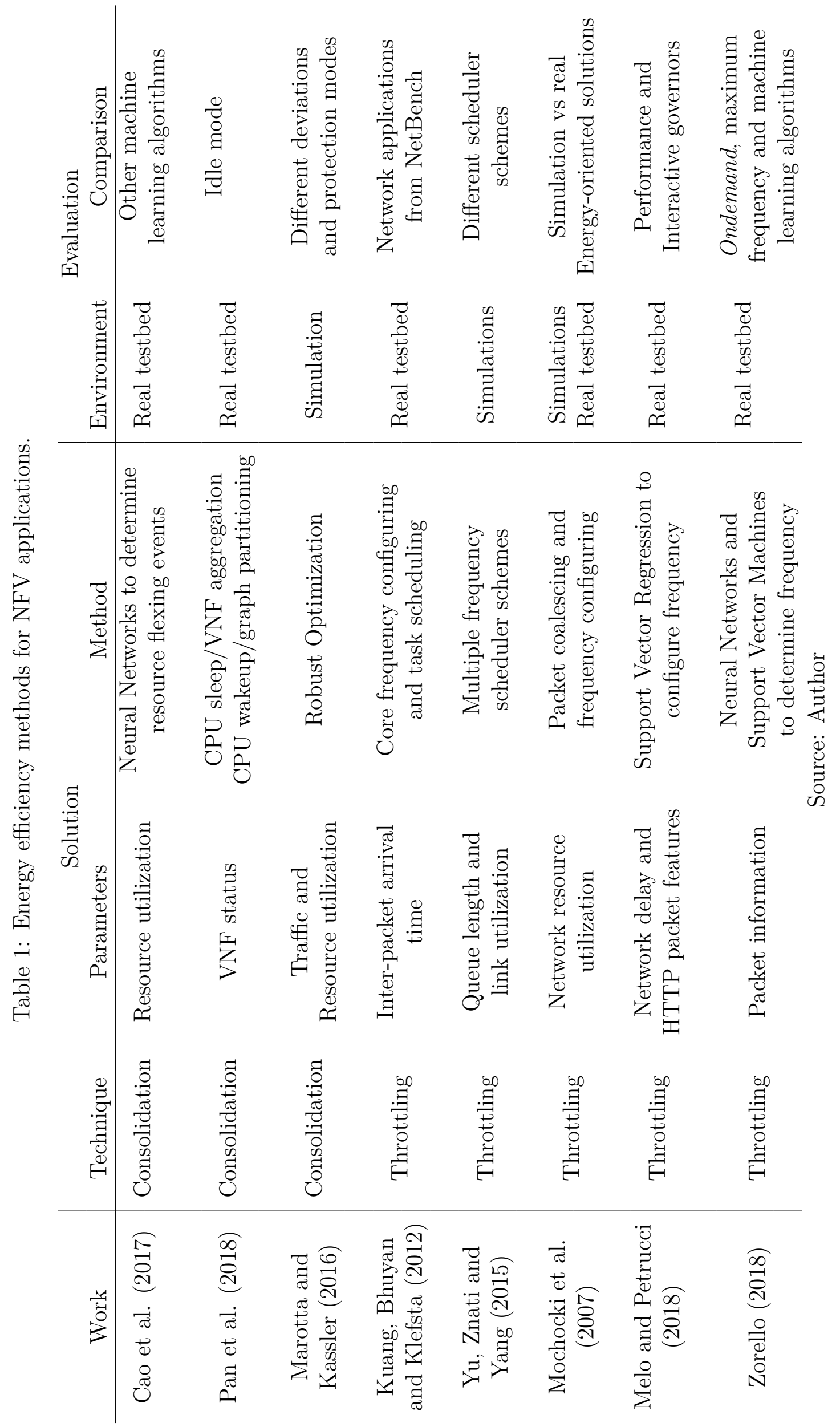




\section{DVFS PREDICTION ENGINE}

This chapter discusses the solution proposed to reduce the energy consumption in Data Centers (DCs). First of all, it is important to describe the method and activities executed in order to propose the solution architecture. Figure 8 illustrates the activities followed in the research method to achieve the objective of proposing a new system architecture based on machine learning that optimizes the frequency selection according to the traffic, enabling reduction of energy consumption in Network Function Virtualization (NFV) applications.

Figure 8: Activities followed to propose a new system architecture.

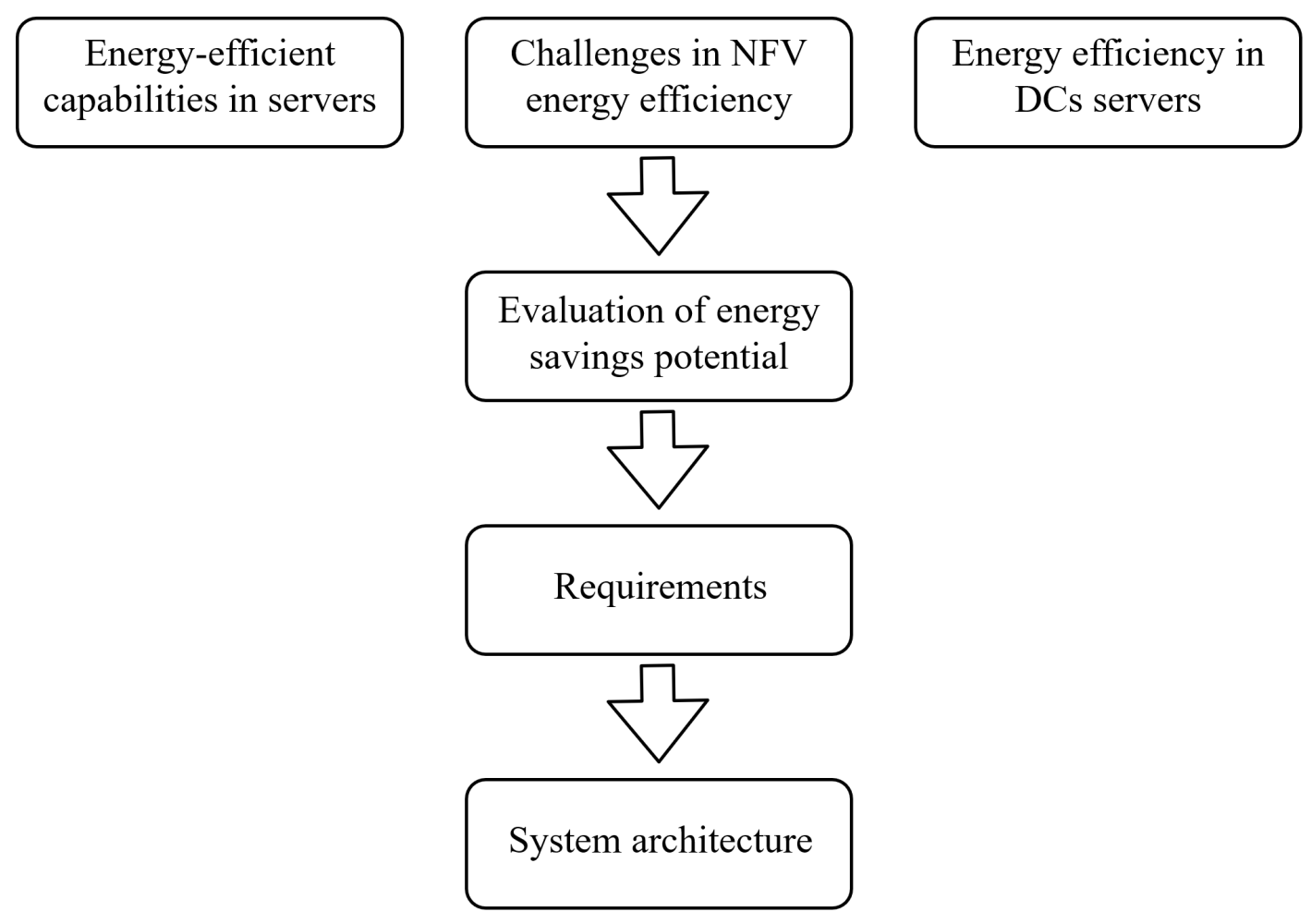

Source: Author

After studying the energy efficiency capabilities available in current processors and the existing solutions to reduce energy consumption in DC servers and NFV applications (refer to Chapter 2 and Chapter 3), a proof of concept was performed to analyze the 
real potential of saving energy when a Virtual Network Function (VNF) is executed. The related work analyses and the investigation of the potential to reduce energy consumption in servers enabled the proposal of the system requirements that should be fulfilled. Following the requirements definition, the architecture could be finally developed.

This chapter is therefore organized as follows: Section 4.1 presents the potential of energy savings when reducing the processors frequency. Section 4.2 then defines the requirements that the architecture must fulfill. Following, Section 4.3 explains the architecture, describing the different modules that compose the proposed solution and the machine learning algorithms that were implemented. Finally, Section 4.4 concludes the chapter.

\subsection{Energy saving potential}

In order to ensure that the high-performance requirements will always be met, Network Function Virtualization (NFV) DC operators hardly ever enable CPU energy efficiency features. Consequently, Dynamic Voltage and Frequency Scaling (DVFS) is usually disabled so that the frequency is set as the maximum and the server is able to process the complete ongoing traffic. This section presents the analysis of the possibility to reduce the server energy consumption when enabling DVFS.

The experiments were run in a server equipped with the processor Intel®Xeon®CPU E3-1230 V2 @3.30GHZ, containing 8 CPUs when HyperThreading is enabled. It contains a RAM of 16GB and has Ubuntu 14.04.5 LTS installed as an operating system. The processor may run in 15 different frequencies that can be controlled by DVFS via the Advanced Configuration and Power Interface (ACPI). The available frequencies start from 1.6 GHz and go up to $3.3 \mathrm{GHz}$. Two Virtual Machines (VMs) were deployed using the open source Linux Kernel Virtual Machine (KVM) hypervisor. One of them is responsible for the traffic generation, while the other one runs the well-known open source Snort@(ROESCH, 1999) Intrusion Detection System (IDS) as an exemplary Virtual Network Function (VNF).

Figure 9 presents the scenarios implemented to understand the energy savings potential when setting lower frequencies.

In the first case, illustrated in Figure 9a, the VMs containing the traffic generator and Snort were deployed in a single server; thus, the Running Average Power Limit (RAPL) driver reports the energy consumed by both VMs. In the second scenario, indicated in 
Figure 9: Testing environment implementation.

(a) Single server.

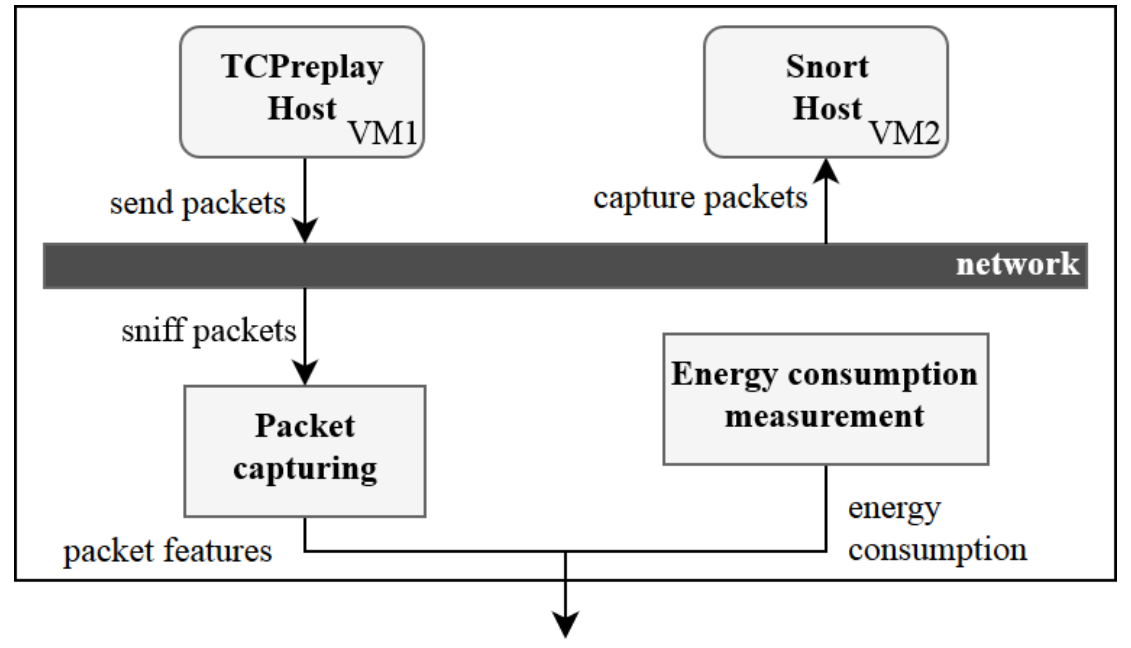

(b) Two servers.

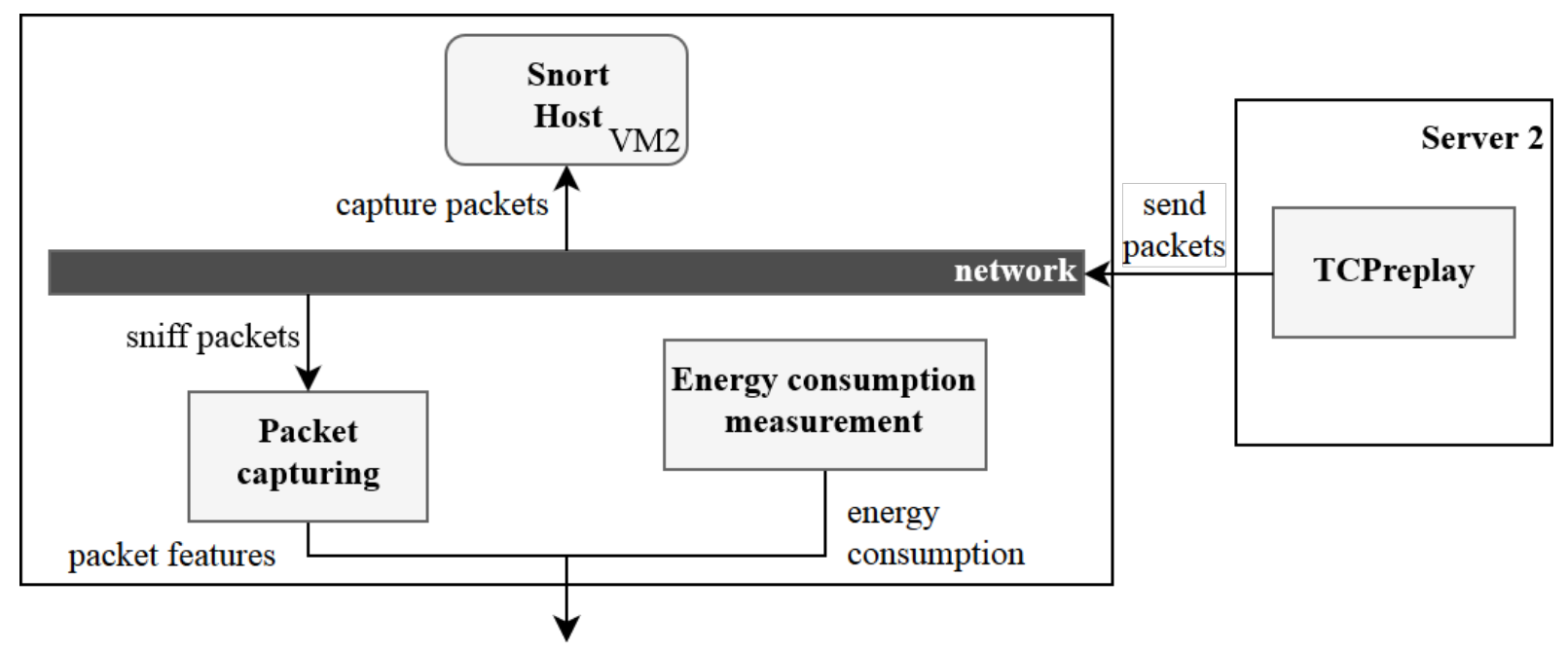

Source: Author

Figure 9b, the traffic generator was implemented in another server, and only the consumption of the VM running Snort was recorded. During this evaluation, the traffic generator transmits the packets at a rate of 9,000 packets per seconds (pps) in both scenarios. This value represents the limit that saturates the CPU to which Snort VM was associated at the lowest frequency, and it is, therefore, ensured that Snort is able to process all packets at any frequency.

TCPreplay Host executes the tcpreplay tool (TURNER, 2014) to replay the traffic from a pcap file generated by Shiravi et al. (2012) into the network. It has already been employed in the literature for Snort performance characterization and consists of a trace without any malicious activity. 
Figure 10 illustrates the energy consumption per transmission rate for five frequencies using a single server and two servers. For readability purposes, it presents only five out of all frequencies measured.

Figure 10: Energy consumption per transmission rate for five frequencies.

(a) Single server.

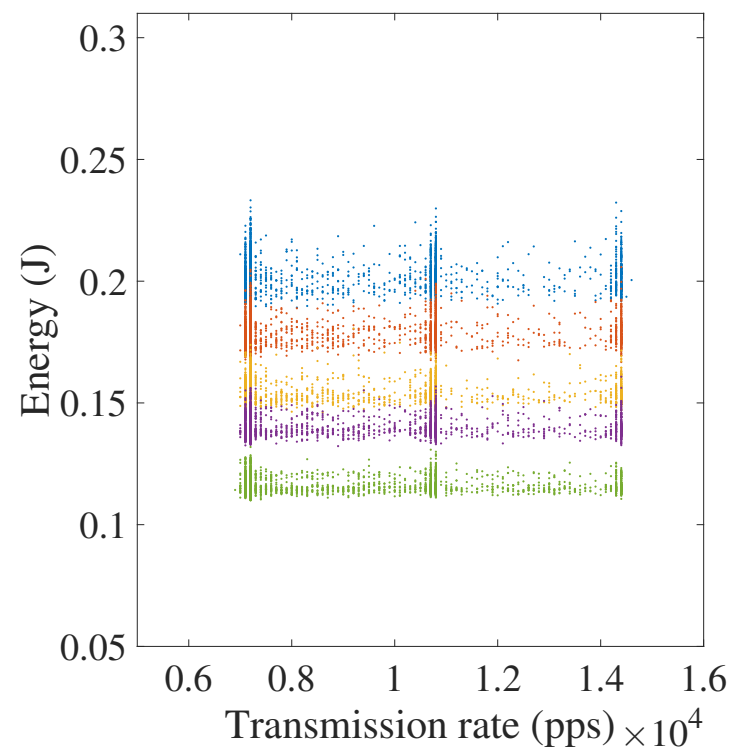

(b) Two servers.

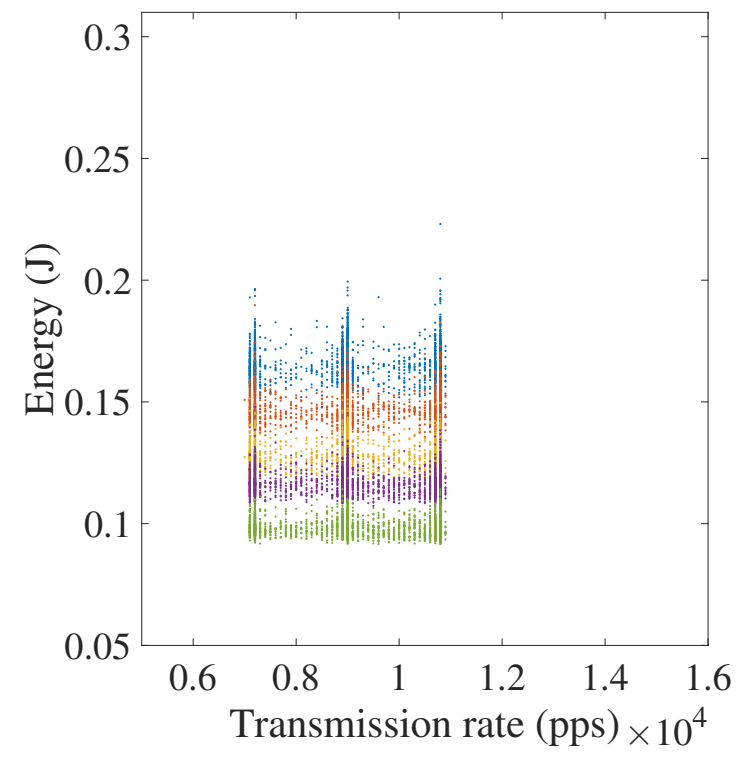

$\bullet 3.3 \mathrm{GHz} \bullet 2.9 \mathrm{GHz} \bullet 2.4 \mathrm{GHz} \bullet 2.1 \mathrm{GHz} \bullet 1.6 \mathrm{GHz}$

Source: Author

Figure 10a and Figure 10b present the energy consumption as a function of the transmission rate per frequency in the scenario with one server and two servers, respectively. It reflects the amount of uncertainty involved with reading the energy consumed by the processor as accumulated over small time intervals (each measurement point represents a $10 \mathrm{~ms}$ time interval).

Figure 11 presents the energy consumed per frequency in the case with one and two servers. Each point in the curves represents the average of all measurement points for every frequency obtained in the experiments.

As anticipated in the literature (SULEIMAN; IBRAHIM; HAMARASH, 2005; SUEUR; HEISER, 2010), there is a quasi-linear increase on the average energy consumption as the frequency is higher. In addition, the energy consumption of a single server is superior to two servers due to the use of a second VM in the same server, which generated more load in the CPU when compared to the two servers scenario, and, consequently, consumes more energy.

Since the normal operating mode in NFV deployment in DCs consists on setting 
Figure 11: Average energy consumption per frequency when the transmission rate is 9,000 pps.

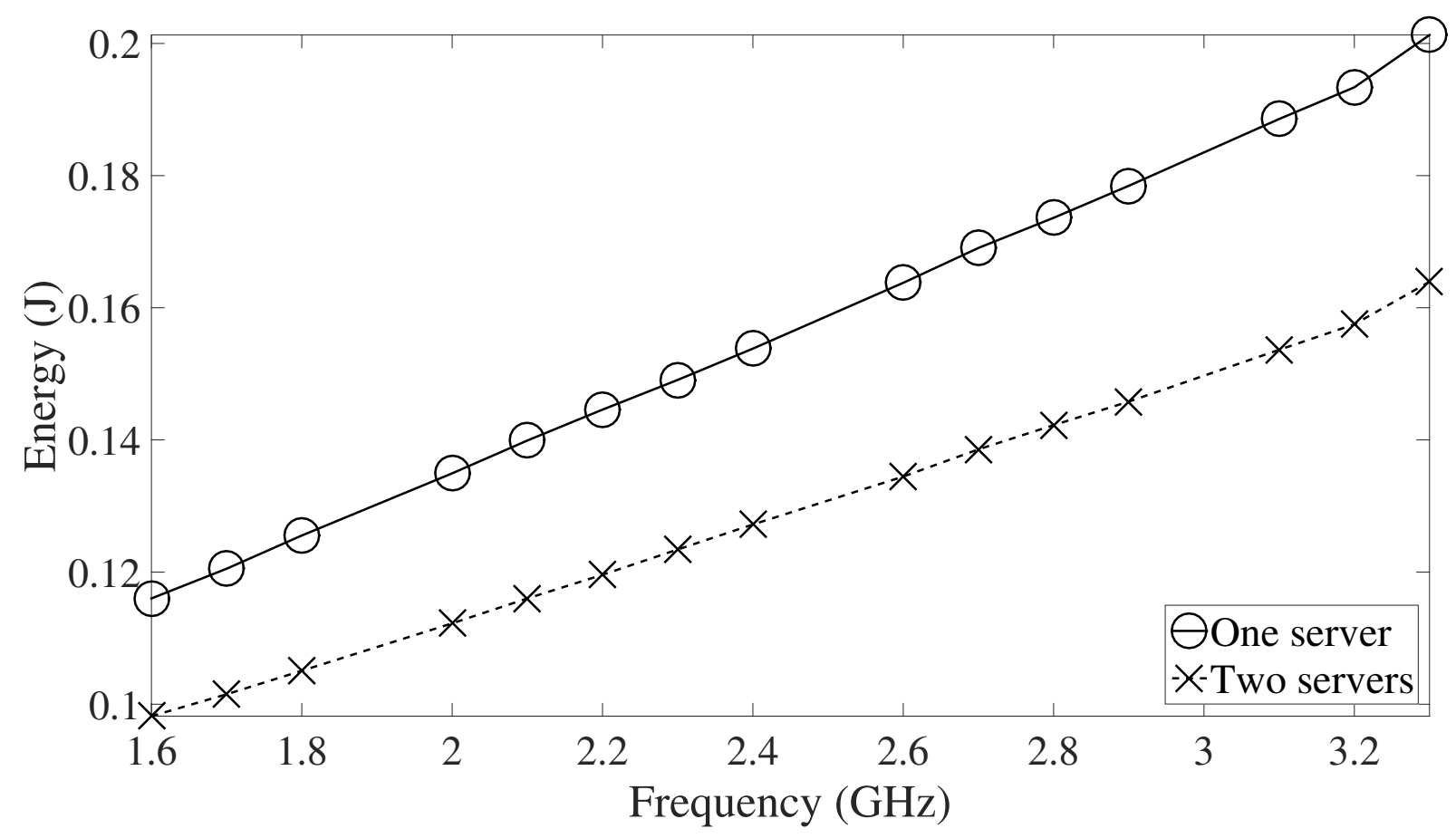

Source: Author

the highest frequency available in the processors, the consumption obtained at $3.3 \mathrm{GHz}$ corresponds to the energy that a typical server would consume in this scenario. Hence, these results show that it is possible to reduce the energy consumption by $42 \%$ or $39 \%$ if lower frequencies were used for the same throughput.

\subsection{Requirements Specification}

After having described the related work and the motivation to provide methods to reduce energy consumption in data center servers, this section exploits the requirements that need to be fulfilled in order to achieve the objective described in Section 1.1, i.e. scale the CPU frequency dynamically using machine learning to enable energy consumption optimization in DCs with NFV applications. In agreement with the works referred in Chapter 2 and Chapter 3, and to the proof of concept in Section 4.1, the Functional Requirements (FR) are described as follows.

FR 1 DVFS framework in the CPU: server throttling should be applied to optimize the core operating voltage and frequency;

FR 2 Network traffic capturing: the network traffic should be sniffed from the Network 
Interface Card (NIC) to evaluate the packets;

FR 3 Packet characteristics evaluation: the network features should be evaluated to determine the CPU operating voltage and frequency, as the energy consumed in servers with NFV applications depends deeply on the packet features (XU et al., 2016);

FR 4 CPU utilization evaluation: the CPU workload related to the packet processing should be analyzed in order to determine the best CPU operating voltage and frequency;

FR 5 Energy performance mode prediction: the cope operating voltage and frequency should be determined according to the momentary traffic and CPU utilization, since it is not possible to know the CPU utilization nor the data throughput beforehand (MAROTTA; KASSLER, 2016);

FR 6 Dynamic scaling: the voltage and frequency should be adapted in a short periodicity (10 ms) as the traffic features are very dynamic;

FR 7 Packet-processing performance: the packet loss should be evaluated to ensure that the energy-efficient technique does not strongly impact the network performance;

FR 8 Simple deployment in different processor architectures: the solution should be easily implemented in a variety of processor architectures, which can have different instructions and energy consumption patterns;

FR 9 Simple transition to other NFV applications: the method should be easily translated to distinct NFV applications that would require different parameters to classify the traffic data;

FR 10 Real environment testing: the experimental results should be executed in servers to understand the real energy savings of the proposed solution instead of simulating the results.

\subsection{System architecture}

This section presents the system architecture that is composed of five modules, as described in Figure 12 (ZORELLO et al., 2018). They are separated in two layers: user and kernel. The kernel level comprises the modules that need permissions to execute 
functions in privileged execution mode (root access), i.e. Network Module, DVFS Module and Energy Consumption Module. The user layer, on the other hand, consists of modules of a higher level that are executed in unprivileged mode, i.e. Machine Learning Module and Prediction Engine Module.

Figure 12: DVFS Prediction Engine Architecture.

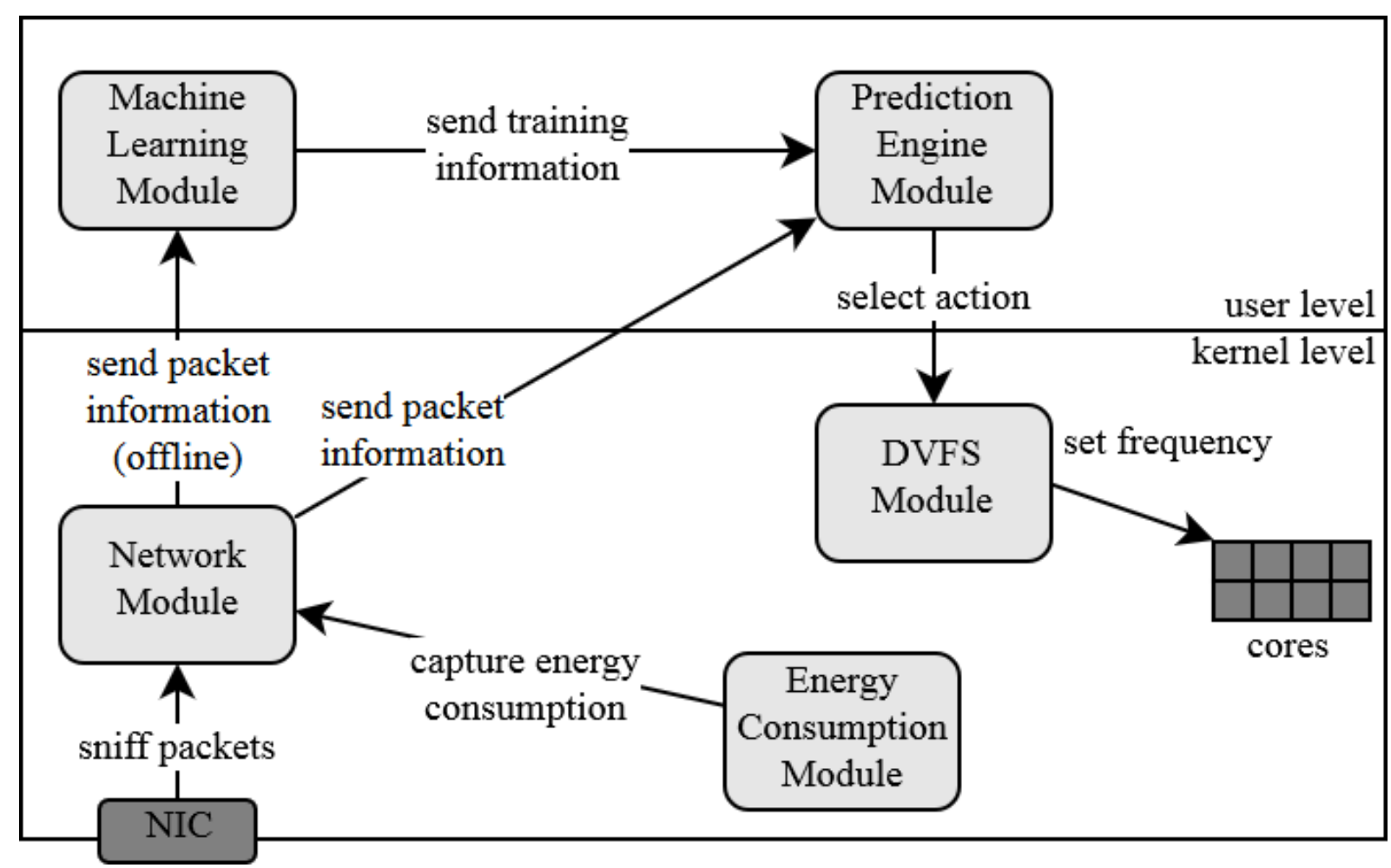

Source: (ZORELLO et al., 2018)

The basic functions of these modules are the following:

- Machine Learning Module: responsible for training the algorithms in the offline phase;

- Prediction Engine Module: executes the classification algorithm in the online phase based on the training from the Machine Learning Module;

- Energy Consumption Module: captures during the offline phase the energy consumption related to the processing of the network ongoing traffic;

- Network Module: extracts the packets features to train the machine learning algorithms in the offline phase and to send to the Prediction Engine Module in the online phase;

- DVFS Module: changes processors frequency. 


\subsubsection{User level modules}

The user level modules contain the components responsible for training and executing the machine-learning algorithm, i.e. Machine Learning Module and Prediction Engine Module.

The Machine Learning Module is active only during the offline phase and is responsible for configuring and training the supervised learning algorithm that will be used during the online phase by the Prediction Engine Module. It aims at building an operational performance profile of the application based on the traffic characterization features, such as TCP, UDP, and number of packets, as expressed in Table 2. Thus, it takes the information from the Network Module regarding the packets and the energy consumption in order to determine the training of the machine learning algorithms.

Table 2: List of traffic characterization features.

\begin{tabular}{|c|c|c|c|c|c|c|c|}
\hline \# & Protocol & $\#$ & Protocol & $\#$ & Protocol & $\#$ & Protocol \\
\hline 1 & HOPOPT & 15 & EMCON & 28 & $\mathrm{RDP}$ & 41 & DHCP \\
\hline 2 & ICMP & 16 & XNET & 29 & IRTP & 42 & НTTP \\
\hline 3 & IGMP & 17 & CHAOS & 30 & ISO-TP4 & 43 & POP3 \\
\hline 4 & GGP & 18 & UDP & 31 & NETBLT & 44 & NETBIOS \\
\hline 5 & IPV4 & 19 & MUX & 32 & MFE-NSP & 45 & IMAP \\
\hline 6 & $\mathrm{ST}$ & 20 & DCN-MEAS & 33 & MERIT-INP & 46 & HTTPS \\
\hline 7 & TCP & 21 & HMP & 34 & DCCP & 47 & $\mathrm{ECHO}$ \\
\hline 8 & $\mathrm{CBT}$ & 22 & PRM & 35 & $3 \mathrm{PC}$ & 48 & MSP \\
\hline 9 & EGP & 23 & XNS-IDP & 36 & IDPR & 49 & TELNET \\
\hline 10 & IGP & 24 & TRUNK-1 & 37 & APLIC & 50 & $\mathrm{BIT}$ \\
\hline 11 & BBN-RCC-MON & 25 & TRUNK-2 & 38 & FTP & 51 & OTHERAP \\
\hline 12 & NVP-II & 26 & LEAF-1 & 39 & SSH & 52 & OTHERPR \\
\hline 13 & PUP & 27 & LEAF-2 & 40 & DNS & 53 & TOTAL \\
\hline 14 & ARGUS & & & & & & \\
\hline
\end{tabular}

Source: Author.

In contrast, the Prediction Engine Module is active only in the online phase. It executes the classification algorithm based on the training of the Machine Learning Module and determines the best frequency according to the inputs coming from the Network Module. It then informs the DVFS Module about the selected frequency.

As discussed in Chapter 1, it is necessary to classify the traffic data into multiple energy performance modes based on a variety of parameters from the network ongoing packets. Therefore the Machine Learning and the Prediction Engine modules are implemented using classification supervised learning algorithms. The objective of supervised 
learning algorithms is to create functions capable of determining the value corresponding to any valid input object after having seen a series of examples (input/output pairs) (GUIDO; MüLLER, 2016). The Artificial Neural Network (ANN) and Support Vector Machines (SVM) are some of the different supervised learning algorithms available in the literature. Both were shown to have high prediction ability with relatively low overhead, presenting good robustness and fitting in a dynamic real-time setting (NEHRU et al., 2013; HEARST et al., 1998). Section 4.3.1.1 and Section 4.3.1.2 describe briefly both algorithms.

\subsubsection{Artificial Neural Networks}

Artificial Neural Networks (ANN) consists of a biologically-inspired algorithm that is able to learn a behavior based on the data it observed previously (NIELSEN, 2015). Kohonen (1988, p. 4) defines ANN as:

\footnotetext{
"'Artificial neural networks' are massively parallel interconnected networks of simple (usually adaptive) elements and their hierarchical organizations which are intended to interact with the objects of the real world in the same way as biological nervous systems do."
}

One of the network structures available is called Multi-Layer Perceptron (MLP) or Sigmoid Neurons, and it is able to classify a set of input and to make decisions based on previously seen examples. It is composed by multiple perceptron layers (NIELSEN, 2015):

- Input layer: encodes the input set;

- Hidden layers: compute the weights to determine the output according to the input;

- Output layer: indicates the algorithm outcome.

When the network receives the input data during the offline phase, it examines the output response to the input pattern to determine the weights in the hidden layer. These values are then used during the online phase to determine the output according to a new input set (GUIDO; MüLLER, 2016).

The MLP used in the Machine Learning Module was implemented with three neuron layers, as Figure 13 illustrates. The first layer is the input layer, where features based 
on the traffic characteristics are presented as stimuli. The last one is the output layer, containing only a single neuron, represents the frequency to be selected. Also, a single hidden layer is used in this implementation.

Figure 13: Neural Network structure for prediction.

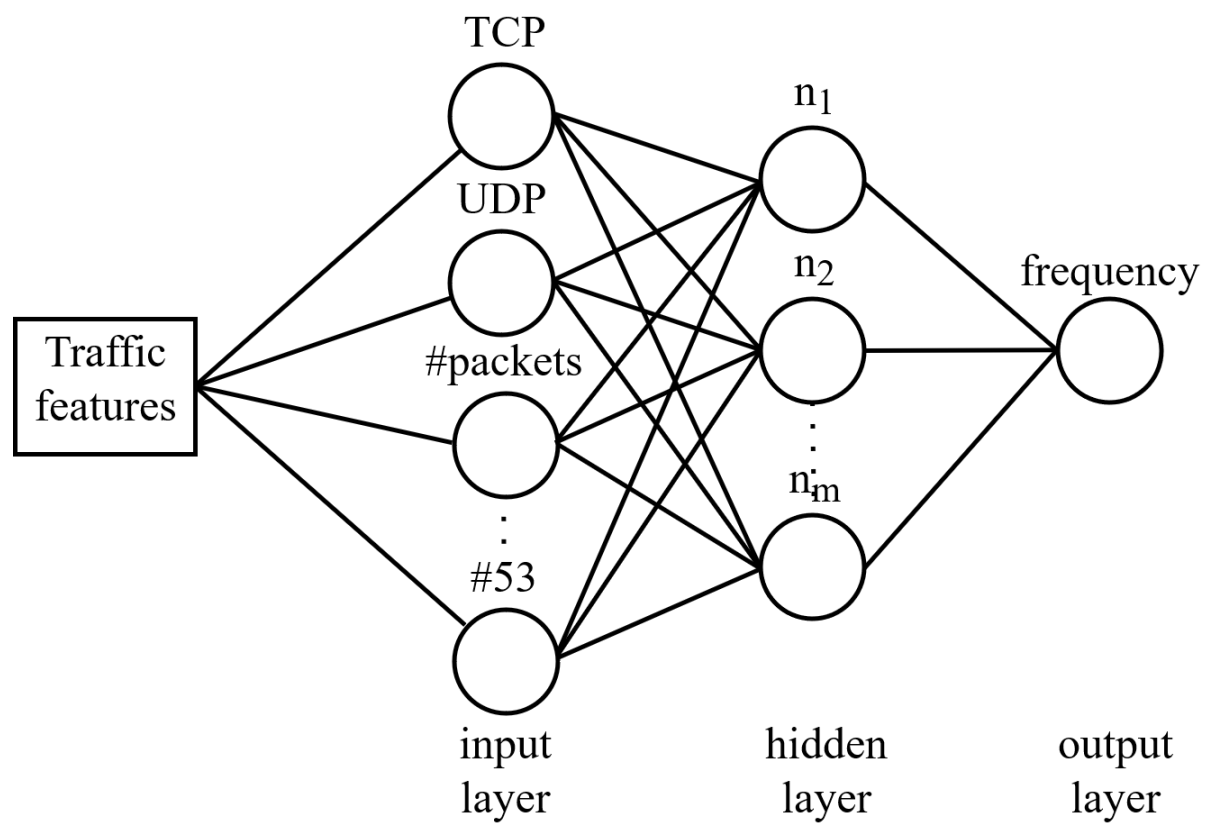

Source: Author

Some parameters can be used to create a training rule in ANN algorithm, such as the number of neurons, $\alpha$ and the batch size.

- Neurons: limits the number of hidden nodes to determine the complexity of the model;

- $\alpha$ : controls the learning rate;

- Batch size: defines the number of samples that are propagated in the neural network.

\subsubsection{Support Vector Machines}

Support Vector Machines (SVM) algorithm was developed according to statistical learning theories and are based on the class separation according to hyperplanes that are constructed by solving constrained optimization problems (HEARST et al., 1998). The simple use of hyperplanes is not, however, enough to solve more complex models (GUIDO; MüLLER, 2016). The use of kernels enables mapping the data into a feature space by computing the scalar product for this space (LORENA; CARVALHO, 2007). Hence, we are able to perform the linear algorithm in this new feature space. 
The Gaussian Kernel in SVM contains the following parameters (GUIDO; MüLLER, 2016):

- C: limits the importance of each point when calculating the support vectors;

- $\gamma$ : controls the width of the kernel.

During training stage, the SVM calculates the support vectors, which are the data points that lie on the border between different classes. Therefore, in this phase, the algorithm learns which are the data points that should be used to compute the decision boundary, i.e. the hyperplane equations (LORENA; CARVALHO, 2007). The classification decision in the online stage is then based on the distance to the support vectors that were calculated (GUIDO; MüLLER, 2016).

The Machine Learning and the Prediction Engine modules using SVM are thus implemented with a Gaussian Kernel to calculate the support vectors that separate the traffic features into different frequencies.

\subsubsection{Kernel level modules}

The Energy Consumption Module is responsible for capturing the energy demand related to the arrival of the packet. To this end, it accesses operating system counters to determine the energy consumed during the amount of time between two consecutive readings. It is active during the training phase in order to generate the data that will be used for training. For evaluation purposes, it is also active during online operations to understand the real energy consumed.

The Network Module is responsible for sniffing packets from the Network Interface Card (NIC) and for extracting features from the ongoing traffic. During the offline phase, i.e. the training of the machine learning algorithm, it examines the packets that are being transmitted in the network towards the virtual network function and creates a database that will be sent to the Machine Learning Module. During the online phase, however, it sends the collected information periodically to the Prediction Engine Module.

The DVFS Module is in charge of configuring the processors frequency. It receives information from the Prediction Engine Module containing the frequency to be set and configures the processor registers accordingly immediately. 


\subsubsection{Workflow}

After having explained the different modules that compose the DVFS Prediction Engine architecture, this section describes the training and the real-time phases workflow, explaining the communication between the modules.

\subsubsection{Training phase workflow}

The diagrams in Figure 14 and Figure 15 illustrate the training workflow. During the offline phase, the Network Module and the Energy Consumption Module work in parallel to capture the data that will be used to train the Machine Learning Module. As explained in Section 4.3.2, the Network Module sniffs the packets from the NIC in order to capture their features, such as the different protocols being transmitted in the network. In the meanwhile, the Energy Consumption Module is measuring the energy consumed by the processor. After a determined timeslot, the Energy Consumption Module sends this information to the Network Module, which adds the energy measurement to the dataset together with the packet features collected during this period. This workflow is repeated until the end of the trace is over. After that, the Network Module transmits the complete dataset to the Machine Learning Module, which is responsible for training the machine learning algorithms that will be used during the online phase.

\subsubsection{Real-time phase workflow}

Figure 16 and Figure 17 present a flow chart and a sequence diagram, respectively, that illustrate the real-time workflow.

During the online phase, the Network Module sniffs the NIC to capture the packets and obtains the packet features periodically. These features are then transmitted to the Prediction Engine Module, which evaluates the information based on the training received from the Machine Learning Module to determine the optimal frequency to be selected. Next, it sends to the DVFS Module the information with the required frequency, and this module will finally set the correct frequency.

For evaluation purposes, the Energy Consumption Module is also active and the packet features are saved in a data set for further investigation. 
Figure 14: Training flow chart.

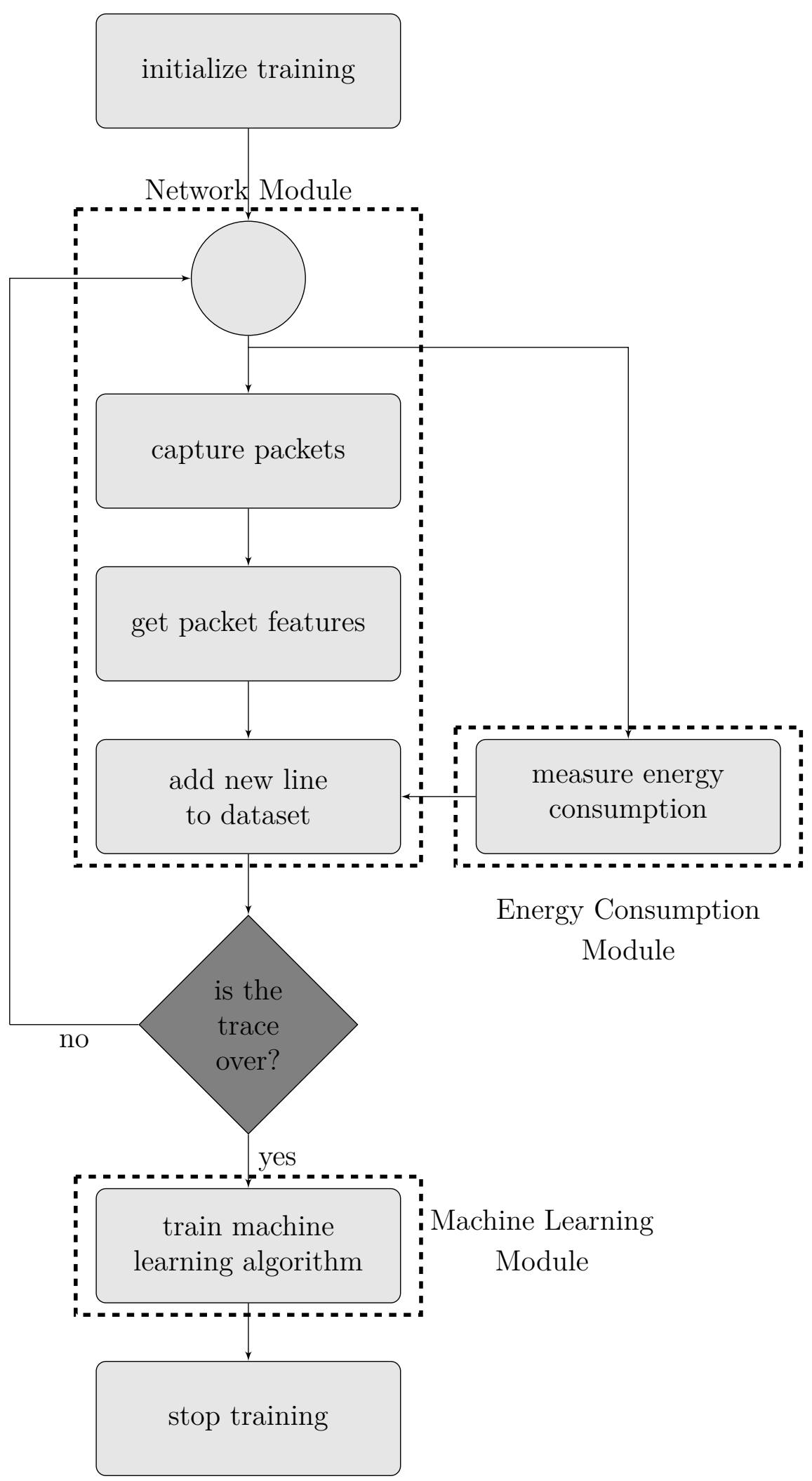

Source: Author. 
Figure 15: Training sequence diagram.

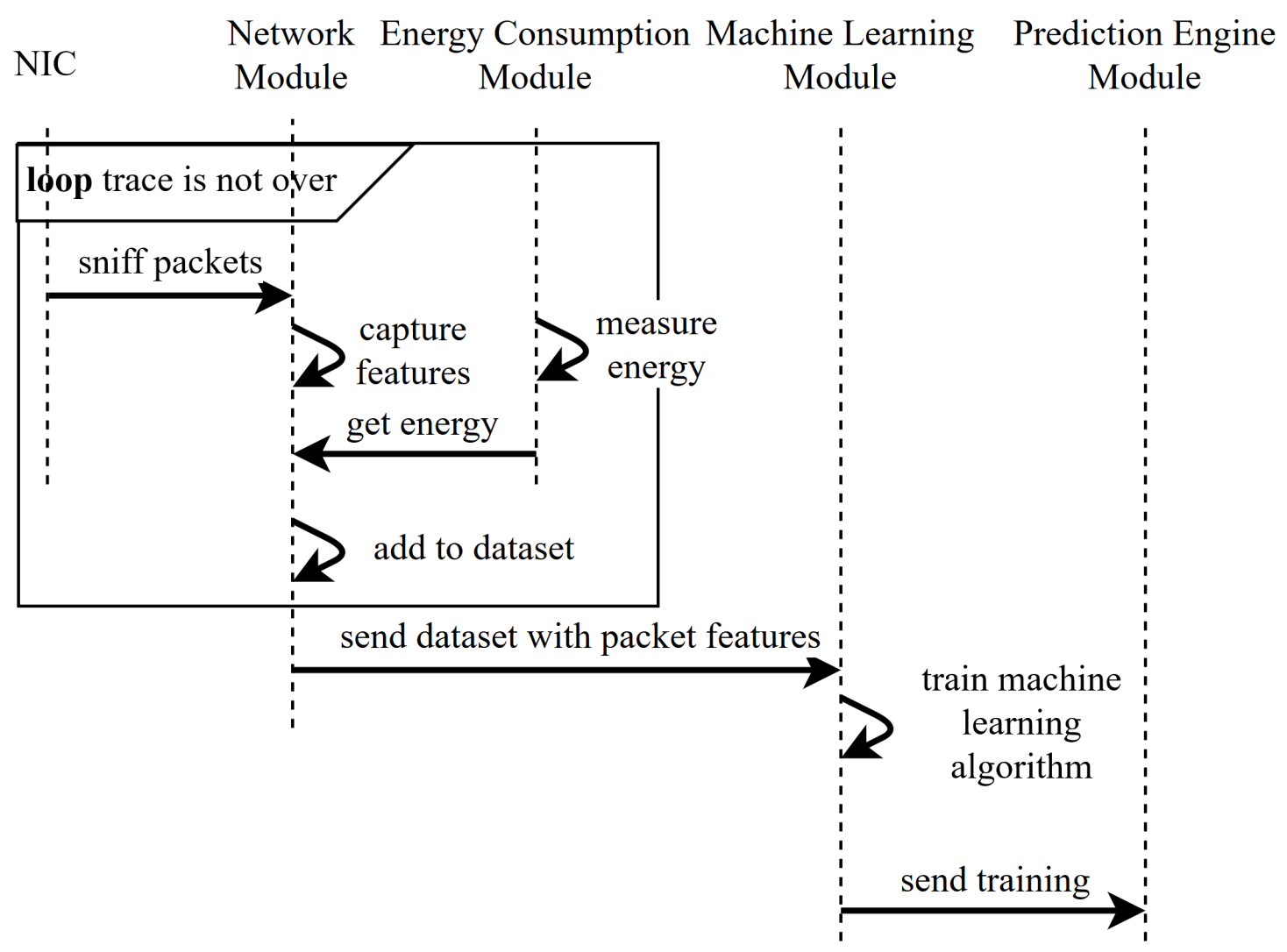

Source: Author.

\subsection{Chapter final remarks}

This chapter initially presents the energy savings potential of using DVFS in servers running NFV applications. Because this type of application requires high performance constraints, operators tend to disable DVFS techniques and, thus, they neglect energy savings. The results in this chapter show that, considering an average transmission rate of $9,000 \mathrm{pps}$, it is possible to reduce the energy consumption in more than $39 \%$.

Based on these results, the DVFS Prediction Engine was developed, whose architecture is also described in this chapter. This architecture is organized in modules that were separated in two layers: user and kernel. The user level comprises the Machine Learning and the Prediction Engine modules, that were implemented with two different machine learning algorithms: Artificial Neural Networks and Support Vector Machines. The kernel level is composed by the components that need administrator power to be executed.

In Chapter 5, the tests architecture of the DVFS Prediction Engine are presented. 
Figure 16: Real-time flow chart.

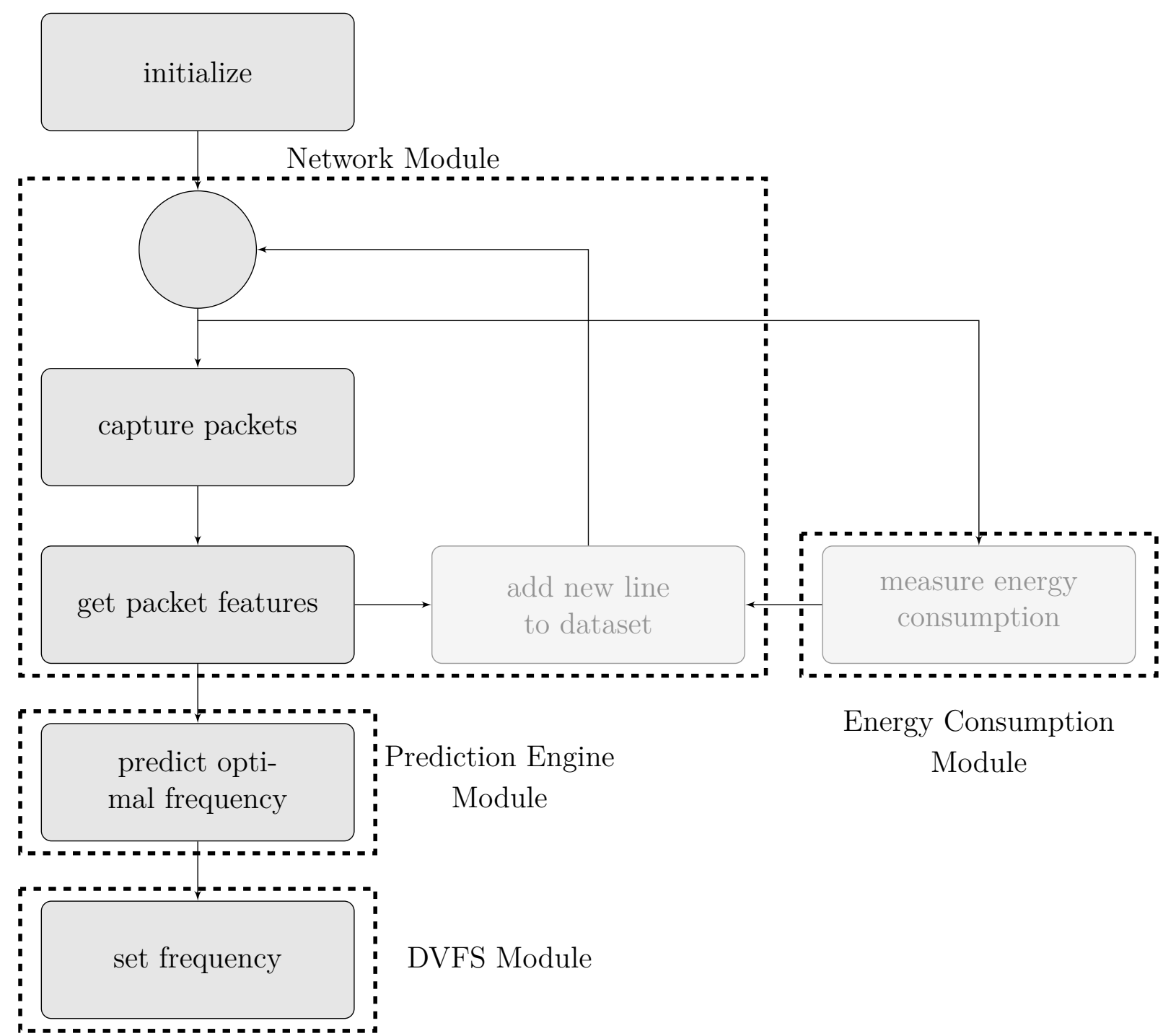

Source: Author. 
Figure 17: Real-time sequence diagram.

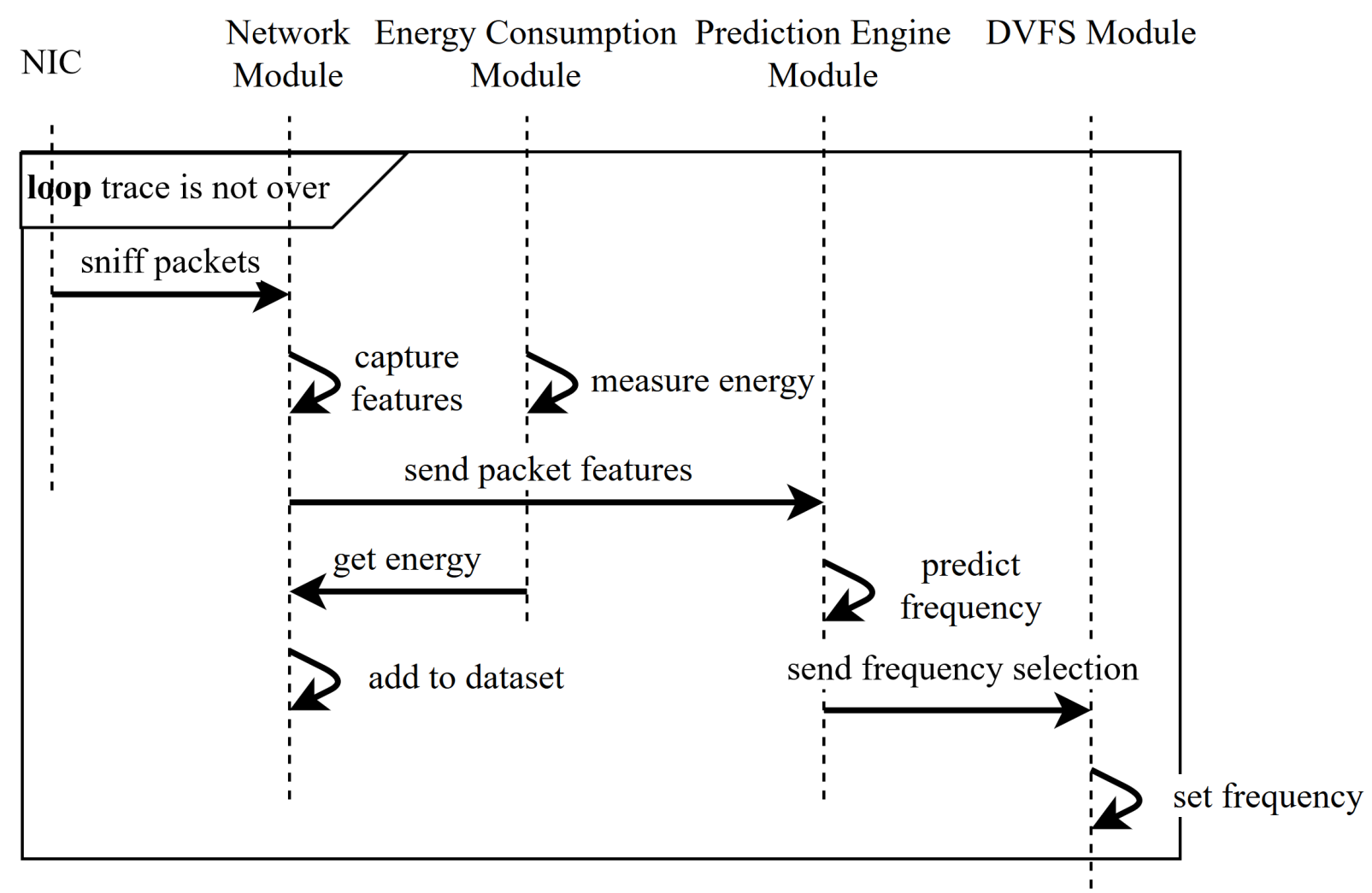

Source: Author. 


\section{IMPLEMENTATION AND RESULTS DESCRIPTION}

This chapter presents DVFS Prediction Engine implementation and the measurement results based on the architecture described in Chapter 4. Section 5.1 describes the testing environment in which the tests were executed. The results were separated in two different sections. First, Section 5.2 discusses the results using a local server with Sandy Bridge CPU microarchitecture and Artificial Neural Networks (ANN) as the machine learning algorithm. This section reports results from both the training and real-time phases. Next, Section 5.3 presents the results when a Haswell CPU microarchitecture is used. This section describes the training and real time phases with ANN and Support Vector Machine (SVM) algorithms. Following, Section 5.4 indicates which requirements from Section 4.2 are fulfilled by the solution proposed in this thesis. Finally, Section 5.5 concludes this chapter.

\subsection{Testing environment}

This section presents the basic implementation of the architecture presented in Section 4.3. For deploying the userspace governor in the processor, the Intel P-State driver was disabled and the Advanced Configuration and Power Interface (ACPI) was enabled. The scenario with a single server hosts two Virtual Machines (VMs) with Ubuntu 14.04 LTS as OS, each one of them running on one exclusively-dedicated core. Figure 18 presents the architecture of the testing environment.

The first VM, namely TCPreplay Host, generates the traffic and sends it to the Virtual Network Function (VNF) that will process it. It runs the tcpreplay tool (TURNER, 2014), which reads a pcap file previously created from real traces and replays it at a determined speed into the network. Thus, it is possible to control the traffic transmission rate via command line parameters and send this traffic to the virtual network to which both VMs are attached. A pcap file containing a trace without malicious activity was employed, 
Figure 18: Testing environment implementation.

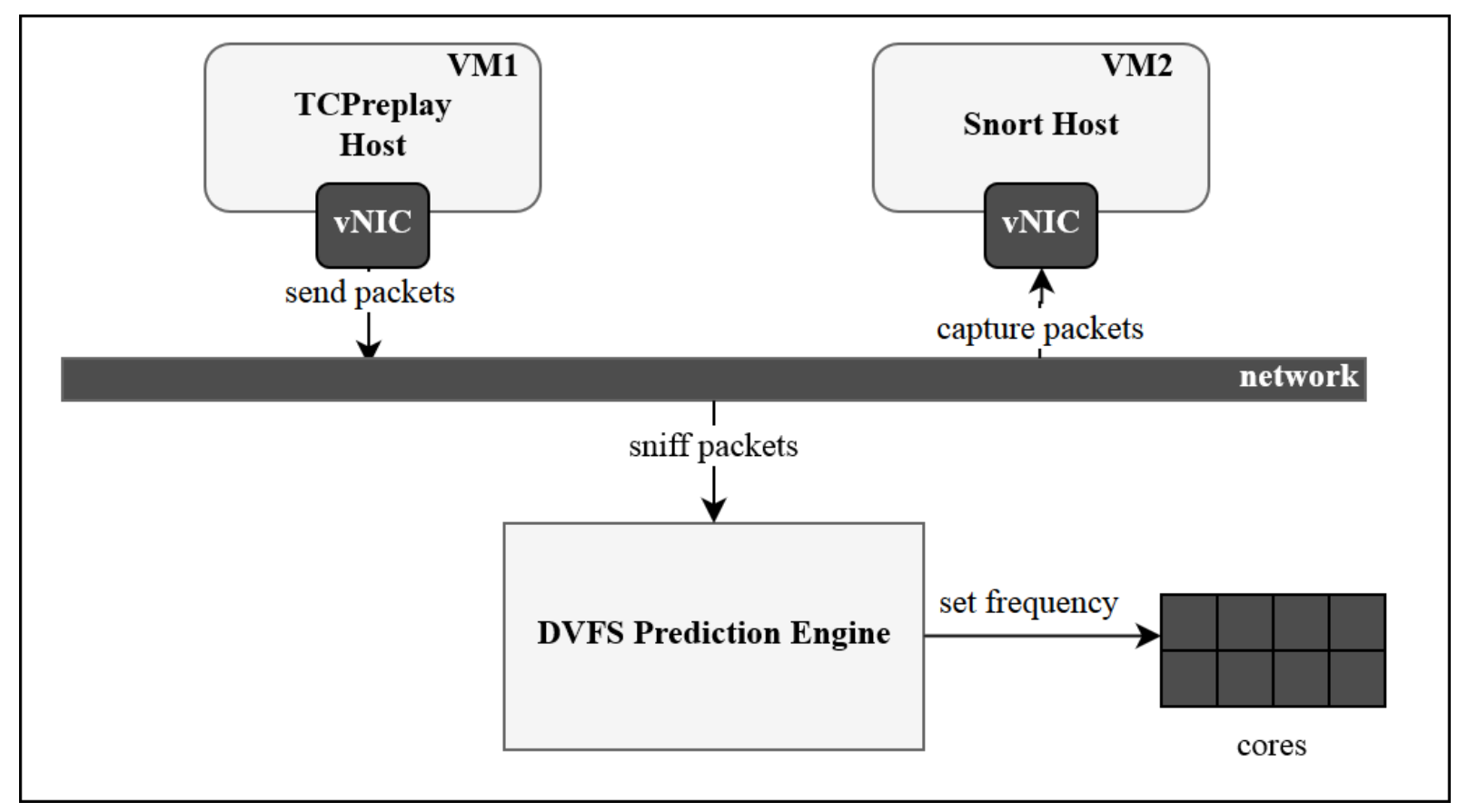

Source: Author

which was provided by the University of New Brunswick (SHIRAVI et al., 2012) and has already been employed in the literature for Snort performance characterization.

The second VM, namely Snort Host, is responsible for executing the VNF instance. In this thesis, Snort@(ROESCH, 1999), a well-known Intrusion Detection System (IDS), was deployed as the VNF which processes the packets from the TCPreplay Host.

Although both VMs are impacted by the change of frequency, the tcpreplay tool is able to generate the traffic efficiently at any frequency, reaching an accuracy of more than $95 \%$ when the transmission rate is set to 100,000 pps in the lowest frequency.

The Dynamic Voltage and Frequency Scaling (DVFS) Prediction Engine module contains the system architecture described in Figure 12, from Chapter 4, Section 4.3. The host machine is responsible for executing it, because of the privileges required to execute some of its functions, such as modifying the frequency and capturing the energy consumption. Its modules were implemented as follow.

The Network Module, responsible for sniffing packets from the Virtual Network Interface Card (vNIC), was implemented in C, using the pcap library (GARCIA, 2008). This implementation provides an Application Programming Interface (API) that allows parsing the data from the incoming traffic and detects different protocols present in the packets up to layer 7 of Open System Interconnection (OSI) stack. Thus, it creates a 
dataset containing 53 features corresponding to the number of times each of the protocols appear.

The Energy Consumption Model, which calculates the energy consumed in the server, was implemented in $\mathrm{C}++$. It gets energy consumption and $\mathrm{CPU}$ cycles values from the Model-Specific Registers (MSR) of the Intel processor through Running Average Power Limit (RAPL) interface.

The Prediction Engine Module, which selects the best frequency according to the training, was implemented in Python, using the well-known scikit-learn library (PEDREGOSA et al., 2011). The Artificial Neural Network (ANN) and Support Vector Machine (SVM) were implemented using 53 features from the Network module as input.

The DVFS module is responsible for setting the processors frequency according to the value determined by the Prediction Engine module. It was implemented in $\mathrm{C}$, using the cpufreq library (BRODOWSKI, 2017), and permits modifying the frequency of all cores according to a pre-configured table containing the available frequencies using the userspace Linux power capping governor.

A number of 5300 data samples was captured in the training stage for each frequency, out of which 5000 data samples were used for training, and 300, for the validation step during the offline learning process in order to understand the training accuracy. The samples were measured at $10 \mathrm{~ms}$ intervals. The length of the time window was chosen arbitrarily, but a number of constraints were taken into account when choosing it. Such constraints included the ability to buffer excess packets that could not be handled due to the classifier determining a too low frequency compared to what would actually be needed for handling the traffic. They also included keeping the energy consumption associated with the classification step itself under control, which meant that the number of times to execute it during one second has to be limited. Other considerations, such as transition times between different CPU frequencies, played a minor role but were double-checked as to not be detrimental.

\subsection{Local server}

The experiments were executed in a server with an Intel®Xeon®CPU E3-1230 V2 @3.30GHz with 16GB RAM. It had 8 CPUs when HyperThreading was enabled and 15 frequency steps that could be controlled by DVFS, from $1.6 \mathrm{GHz}$ to $3.3 \mathrm{GHz}$. It had Ubuntu 14.04 LTS installed as the Operating System (OS). 


\subsubsection{Training results}

During the offline phase, a dataset was built to train the Machine Learning Module. To collect this data, TCPreplay Host sent packets repeatedly from the same traffic trace, but at different transmission rates according to the frequency, so that it induces more than $90 \%$ utilization at every frequency in the processor core that executes Snort. Therefore, the maximum throughput that the testbed cores can handle for each frequency was estimated.

Table 3 indicates the transmission rate selected for each frequency. It illustrates the fact that, the throughput that can be supported by the VNF is higher as the frequency is increased. Indeed, the transmission rate when the processors frequency is set to $3.3 \mathrm{GHz}$, i.e. the highest, is twice the one for $1.6 \mathrm{GHz}$, i.e. the lowest.

Table 3: Transmission rate selected per frequency for training with local server.

\begin{tabular}{ccc}
\hline$\#$ & Frequency $(\mathrm{GHz})$ & Throughput $(\mathrm{pps})$ \\
\hline 1 & 3.30 & 19,000 \\
2 & 3.20 & 17,500 \\
3 & 3.10 & 16,500 \\
4 & 2.90 & 15,500 \\
5 & 2.80 & 15,000 \\
6 & 2.70 & 14,500 \\
7 & 2.60 & 13,800 \\
8 & 2.40 & 13,000 \\
9 & 2.30 & 12,700 \\
10 & 2.20 & 12,200 \\
11 & 2.10 & 11,500 \\
12 & 2.00 & 11,000 \\
13 & 1.80 & 10,500 \\
14 & 1.70 & 9,800 \\
15 & 1.60 & 9,000 \\
\hline \multicolumn{3}{c}{ Source: Author. }
\end{tabular}

Figure 19 and Figure 20 display the energy consumption, in joules $(\mathrm{J})$, and the number of cycles, respectively, executed as a function of the rate in which packets arrive, in packets per second (pps), for all frequencies. These graphs show that the increase of the frequency value leads to the execution of more cycles and, therefore, a superior energy consumption. Furthermore, when considering a single frequency, the transmission rate varies significantly, because the TCPreplay tool ensures only the average transmission rate; thus, it alters the speed regularly.

Observing these graphs, it is clear that close frequencies overlap others in terms of the energy consumed and the number of cycles executed in the period of $10 \mathrm{~ms}$. Introducing 
this dataset to train the Machine Learning Model could generate errors, as the Prediction Engine Module would not be able to distinguish frequencies that have similar energy consumption and number of cycles being executed. The accuracy when training the Machine Learning Module with 50 neurons was considered to be unsuitable, as the value was approximately $71 \%$ according to the offline validation step executed with part of the data used for training, as presented in Table 4a. Table 9a presents all parameters tested to train the ANN algorithm.

To avoid having such low accuracy percentages, a subset of eight instead of fifteen frequencies was used during the offline phase, as presented in Figure 21 and Figure 22. By training the Machine Learning Model with this dataset, the accuracy reached a value more than $25 \%$ higher, arriving at $76.4 \%$ and $76.9 \%$ when selecting 20 and 200 neurons in the hidden layer, respectively, as shown in Table 4a.

Table 4 presents only the highest accuracy values obtained. Refer to Appendix A for the other parameters result.

Table 4: Highest accuracy values obtained for each set of ANN parameters in local server.

(a) Fifteen frequencies.

\begin{tabular}{cccc}
\hline Neurons & $\alpha$ & Batch & Accuracy \\
\hline 50 & 0.001 & 200 & $71 \%$ \\
50 & 0.001 & 400 & $70.6 \%$ \\
50 & 0.001 & 600 & $70.6 \%$ \\
50 & 0.01 & 600 & $70.6 \%$ \\
100 & 0.001 & 200 & $70.6 \%$ \\
100 & 0.01 & 200 & $71.1 \%$ \\
200 & 0.001 & 200 & $70.8 \%$ \\
200 & 0.001 & 600 & $71.8 \%$ \\
200 & 0.01 & 600 & $70.1 \%$ \\
400 & 0.001 & 200 & $70.2 \%$ \\
\hline
\end{tabular}

(b) Eight frequencies.

\begin{tabular}{cccc}
\hline Neurons & $\alpha$ & Batch size & Accuracy \\
\hline 10 & 0.001 & 200 & $76 \%$ \\
10 & 0.001 & 400 & $76.1 \%$ \\
10 & 0.01 & 200 & $76.1 \%$ \\
10 & 0.01 & 400 & $76.2 \%$ \\
20 & 0.001 & 200 & $76 \%$ \\
20 & 0.001 & 600 & $75.9 \%$ \\
20 & 0.01 & 200 & $76.4 \%$ \\
20 & 0.01 & 400 & $75.7 \%$ \\
50 & 0.001 & 200 & $75.7 \%$ \\
200 & 0.001 & 200 & $76.9 \%$ \\
\hline
\end{tabular}

Source: Author

\subsubsection{Real-time results}

In the real-time scenario, a different trace provided by Santanna et al. (2015) was used. The system extracts the same features and traffic statistics with which the Machine Learning Model was trained, i.e. the types of protocol and number of packets per protocol. The Prediction Engine Module receives in real-time an array of 53 features containing protocol information every $10 \mathrm{~ms}$. It analyzes them and uses the learning acquired in 
Figure 19: Energy consumption per number of packets received for fifteen frequencies from $1.6 \mathrm{GHz}$ to $3.3 \mathrm{GHz}$.

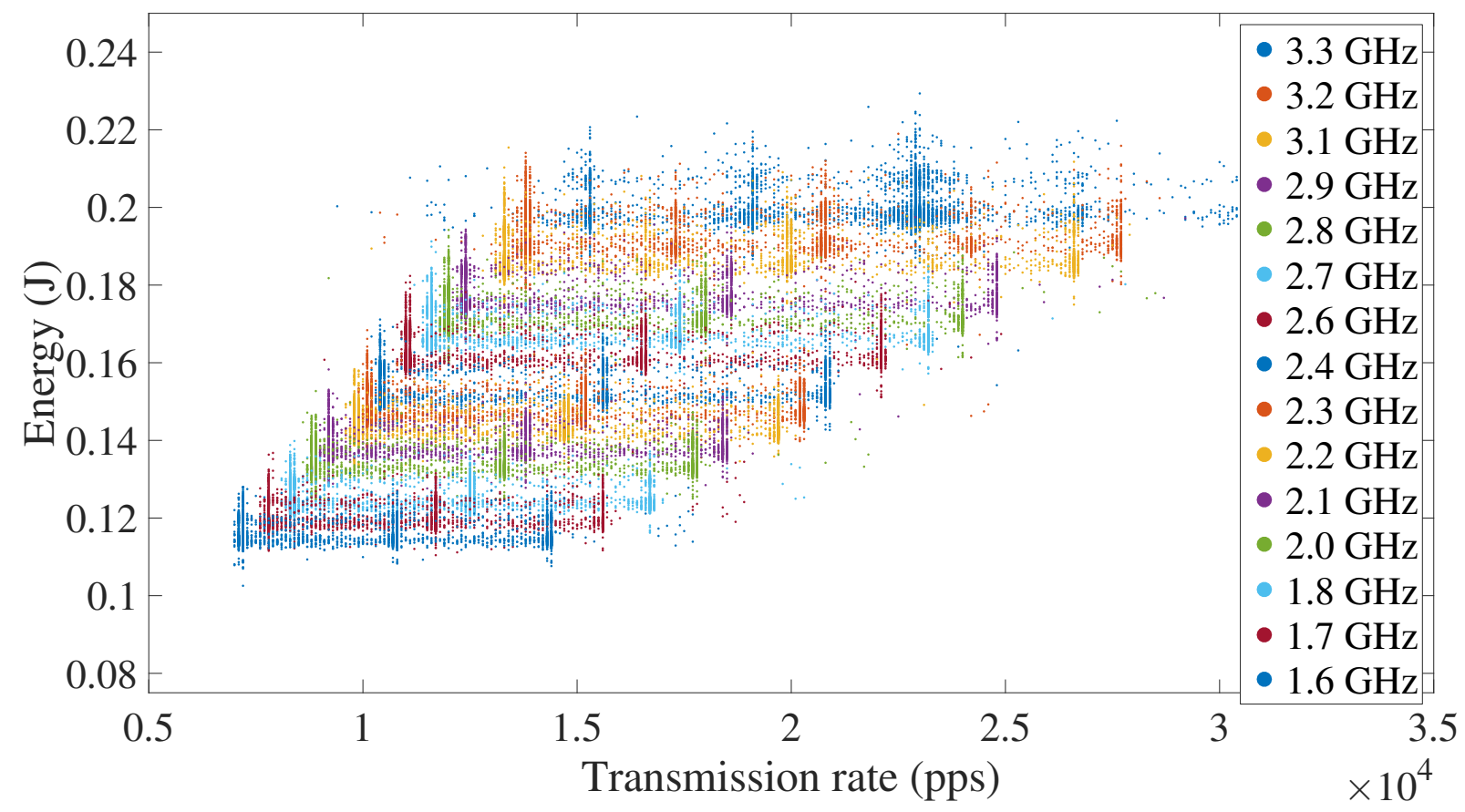

Source: Author

Figure 20: Number of cycles executed per number of packets received for fifteen frequencies from $1.6 \mathrm{GHz}$ to $3.3 \mathrm{GHz}$.

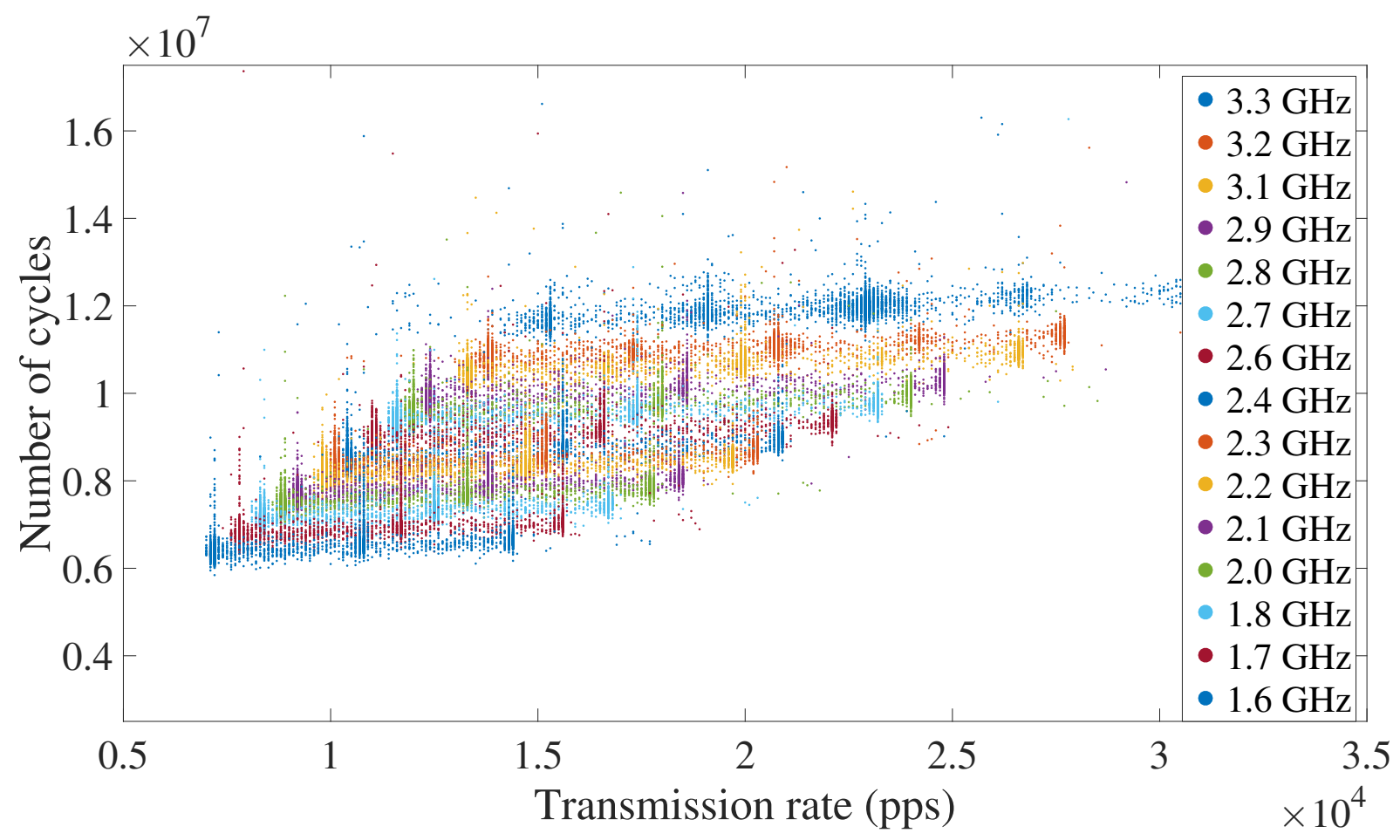

Source: Author 
Figure 21: Energy per number of packets received for eight frequencies from $1.6 \mathrm{GHz}$ to $3.3 \mathrm{GHz}$.

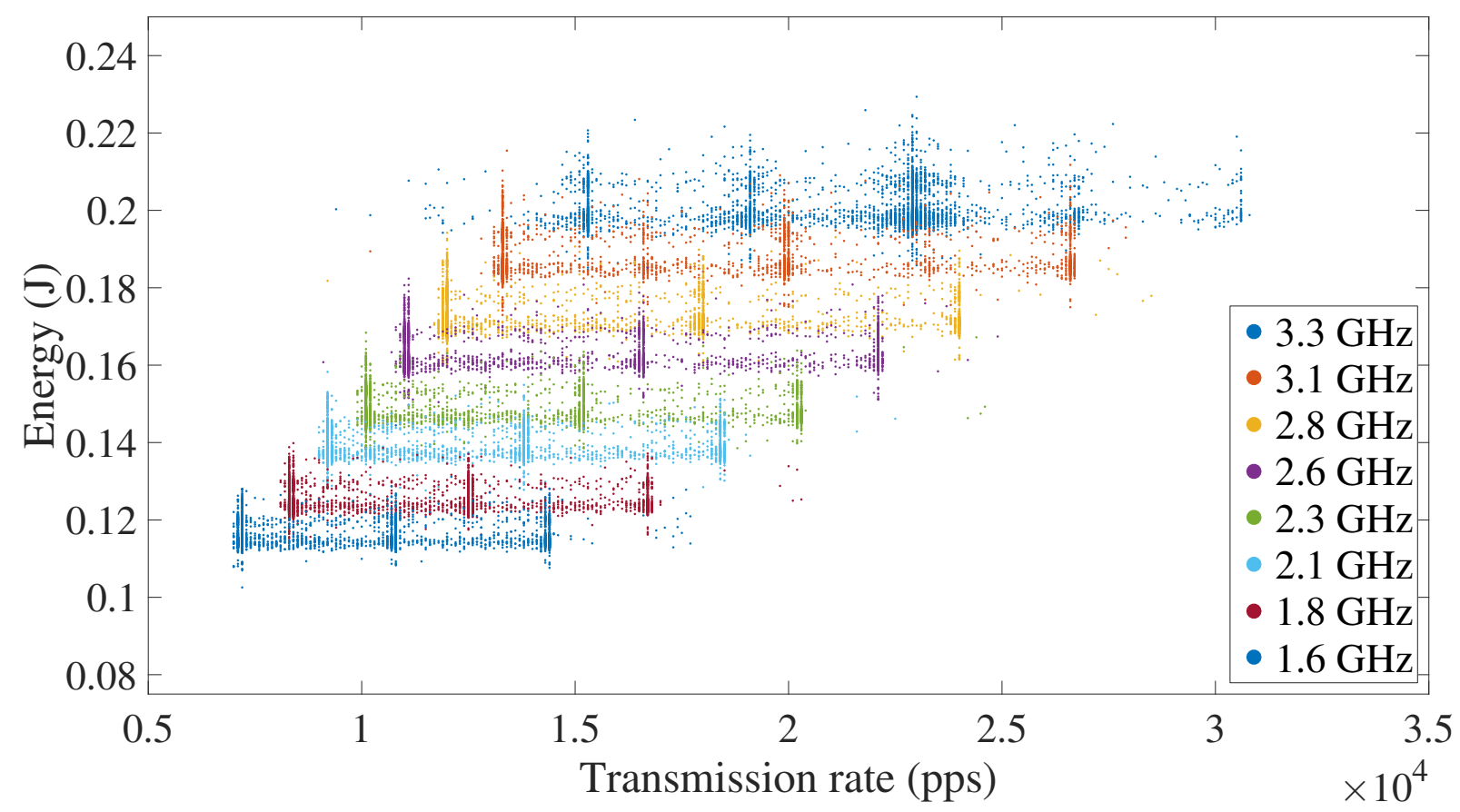

Source: Author

Figure 22: Cycles executed per number of packets received for eight frequencies from 1.6 $\mathrm{GHz}$ to $3.3 \mathrm{GHz}$.

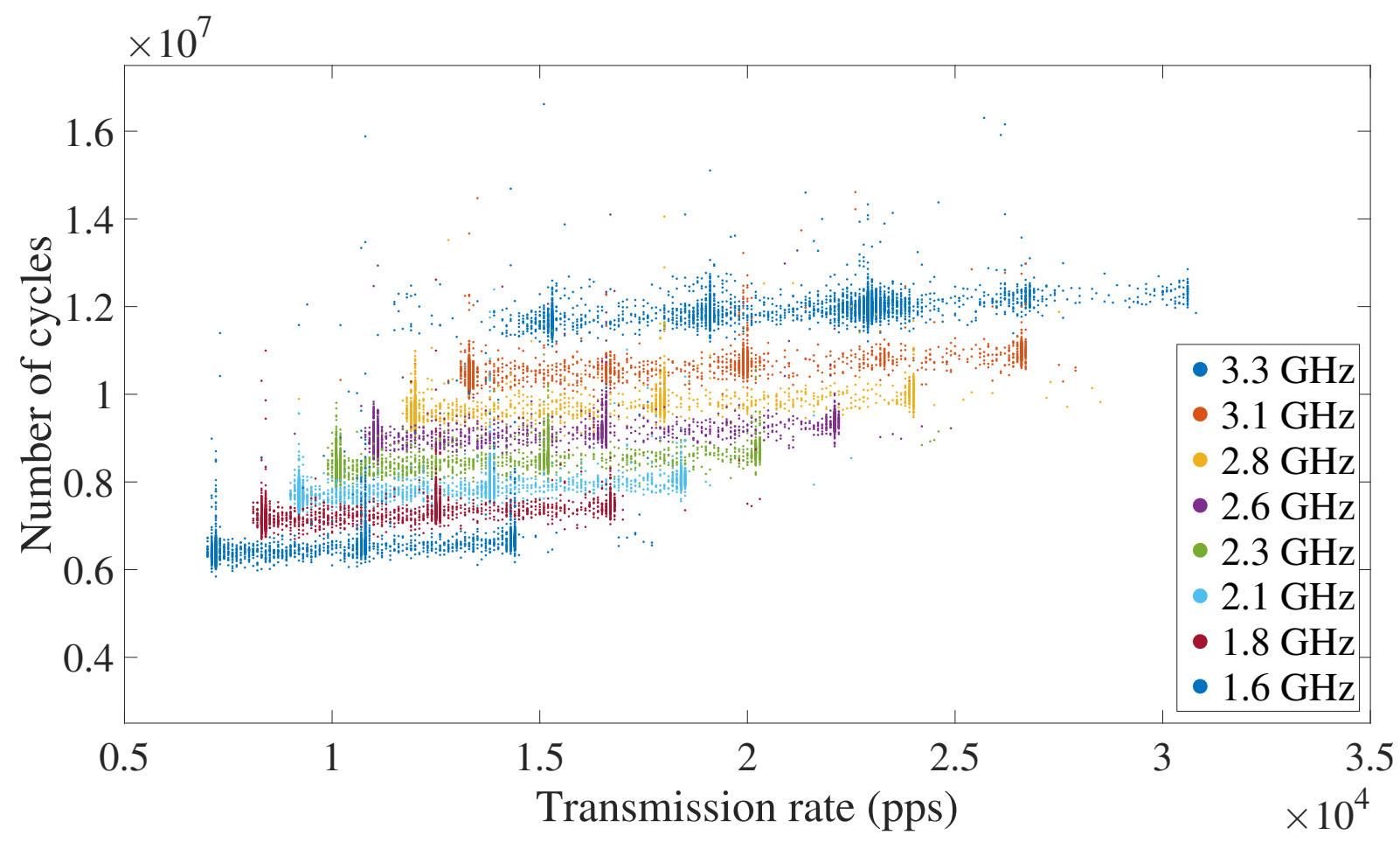

Source: Author 
the Machine Learning Model during the training stage to estimate the best frequency. Depending on the frequency selected by the Prediction Engine Module, the DVFS Module adjusts immediately the frequency of the processors.

As presented in Section 5.2.1, it is possible to increase the accuracy in the prediction when the neural network is trained with 200 neurons instead of 20. Consequently, it would permit the Prediction Engine Model to select the correct frequency more often and, thus, reduce more the energy consumption. However, the use of a larger number of neurons creates a greater complexity in the Prediction Engine, which leads to an overhead that contributes to higher energy consumption. The measurements of the average energy consumption as a function of the transmission rate for 20 and 200 neurons shown in Figure 23 confirm this.

Figure 23: Energy consumption per transmission rate with 20 and 200 neurons.

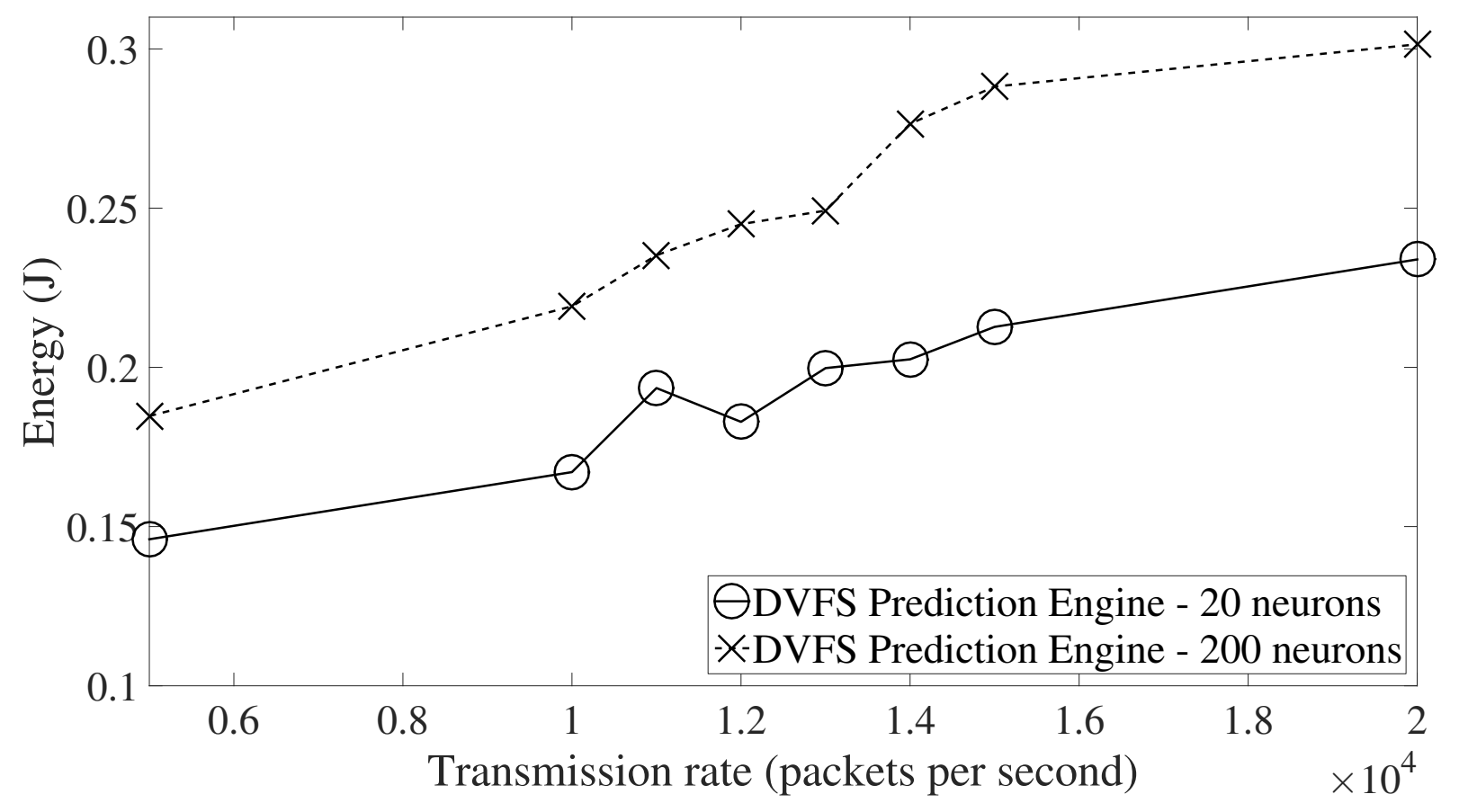

Source: Author

Regardless of the transmission rate, the energy consumption when ANN has 20 neurons in the hidden layer is always lower. Hence, the results presented in this section consider the Prediction Engine Model with 20 neurons, which is then compared to the other two scenarios: maximum frequency and ondemand governor.

From Figure 23, it is verified that the energy consumption when using the DVFS Prediction Engine increases almost linearly as the transmission rate specified in TCPreplay Host is faster using 20 or 200 neurons. This reduction in energy efficiency is a consequence 
of the selection of upper frequencies when the traffic is more intense in order to ensure the packet processing performance. Thus, an interesting evaluation is to compare the results considering the three scenarios - DVFS Prediction Engine, maximum frequency, and ondemand governor - for different transmission rates in order to understand whether DVFS Prediction Engine is able to reduce energy consumption.

Figure 24 presents the energy consumption measurements in time when the transmission rate is set to 10,000 pps in the TCPreplay VM.

Although presenting the highest standard deviation, the scenario using the DVFS Prediction Engine is the lowest energy consuming, as its average value represent a reduction of approximately $15 \%$ when compared to the maximum frequency scenario, and $27 \%$ with respect to the ondemand governor. Its significant standard deviation $-0.032 \mathrm{~J}$ against $0.014 \mathrm{~J}$ for maximum frequency and ondemand governor scenarios - is due to the high rate in which the processors frequency is changed. Because the DVFS Prediction Engine analyses the information sent by the Network Module every $10 \mathrm{~ms}$ and the traffic rate is not steady, it can promptly change from a frequency in which the processor consumes less energy to another more expending to ensure that Snort is able to process all packets.

Figure 25 shows the energy efficiency - defined as the energy needed to process the packets received, expressed as energy per bit $(\mathrm{J} / \mathrm{bit})$ - as a function of the rate in which packets are received in the network.

As expected from the results of Figure 25, the energy efficiency of DVFS Prediction Engine is the best among the three scenarios, as it always presents the lowest value. Therefore, the DVFS Prediction Engine consumes less energy for the same amount of packets being processed when compared to the other scenarios.

Figure 26 and Figure 27 present the same measurements, but at a higher transmission rate, 15,000 pps. As explained previously, the scenario using a prediction engine consumes more energy as the transmission is higher because upper frequencies need to be used in order to provide the required performance. Figure 26 and Figure 27 illustrate this effect, as they show the energy consumption measurements in time and the energy efficiency for all three scenarios, respectively.

Because the prediction engine consumes more as the traffic increases, Figure 26 shows that it surpasses the energy consumption of the maximum frequency scenario at 15,000 pps, with an average of $0.221 \mathrm{~J}$ against $0.174 \mathrm{~J}$. The results from Figure 26 and Figure 27 indicate that there is a reduction in the energy efficiency of the system using DVFS Prediction Engine when compared to the maximum frequency. 
Figure 24: Energy consumption in time for DVFS Prediction Engine, maximum frequency and ondemand governor at 10,000 pps.

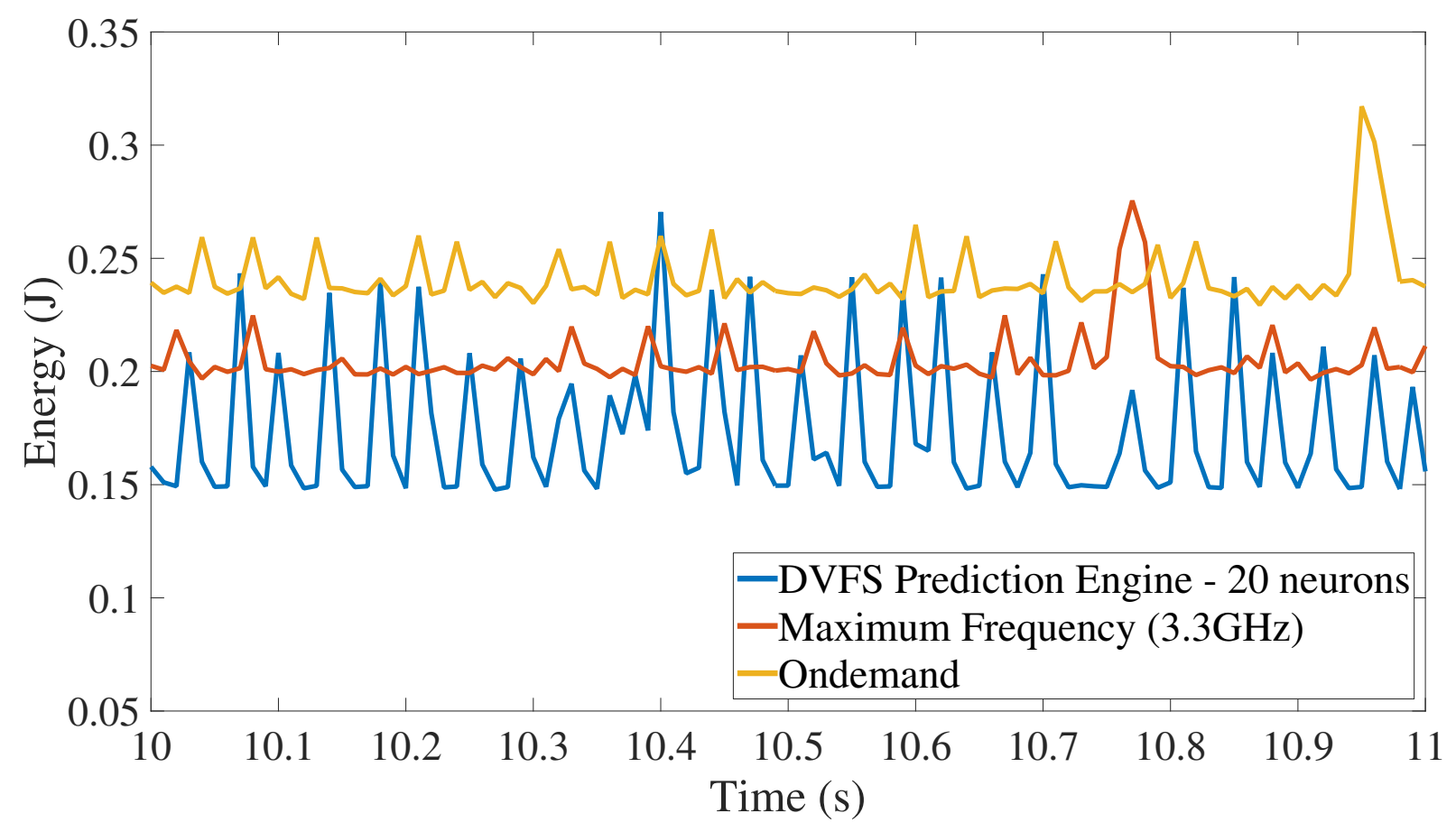

Source: Author

Figure 25: Energy efficiency per transmission rate for DVFS Prediction Engine, maximum frequency and ondemand governor at 10,000 pps.

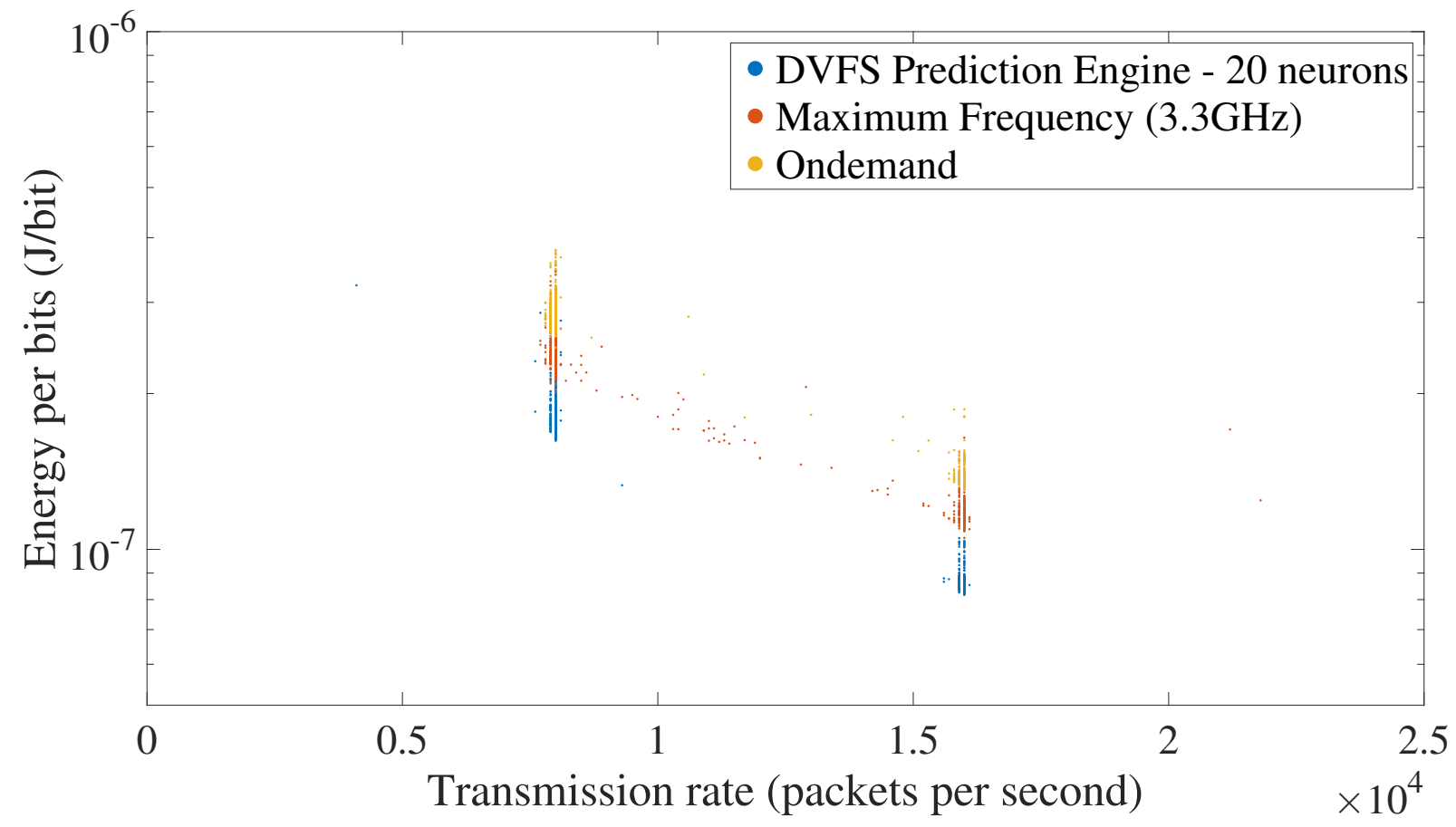

Source: Author 
Figure 26: Energy consumption in time for DVFS Prediction Engine, maximum frequency and ondemand governor at 15,000 pps.

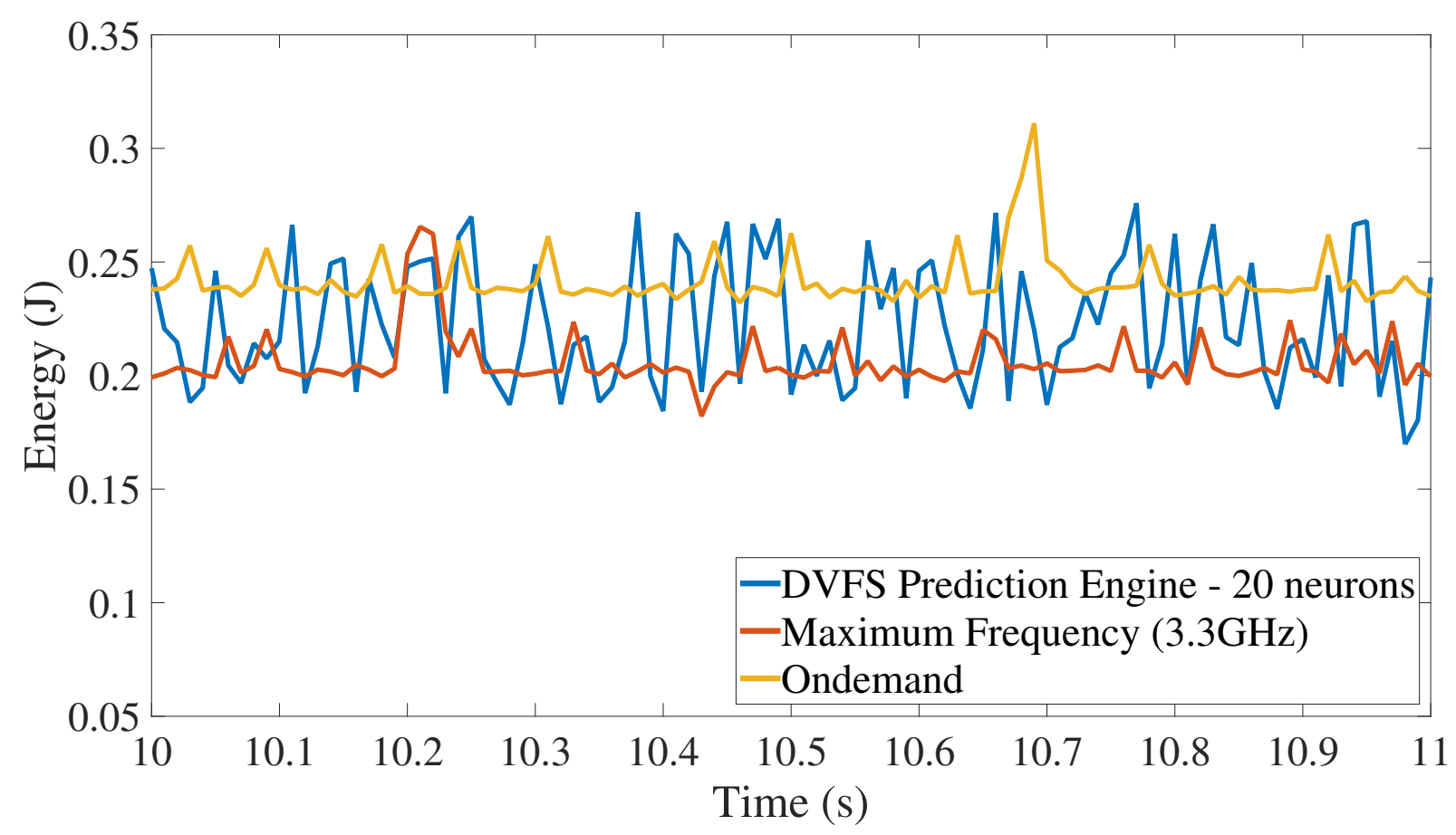

Source: Author

Figure 27: Energy efficiency per transmission rate for DVFS Prediction Engine, maximum frequency and ondemand governor at 15,000 pps.

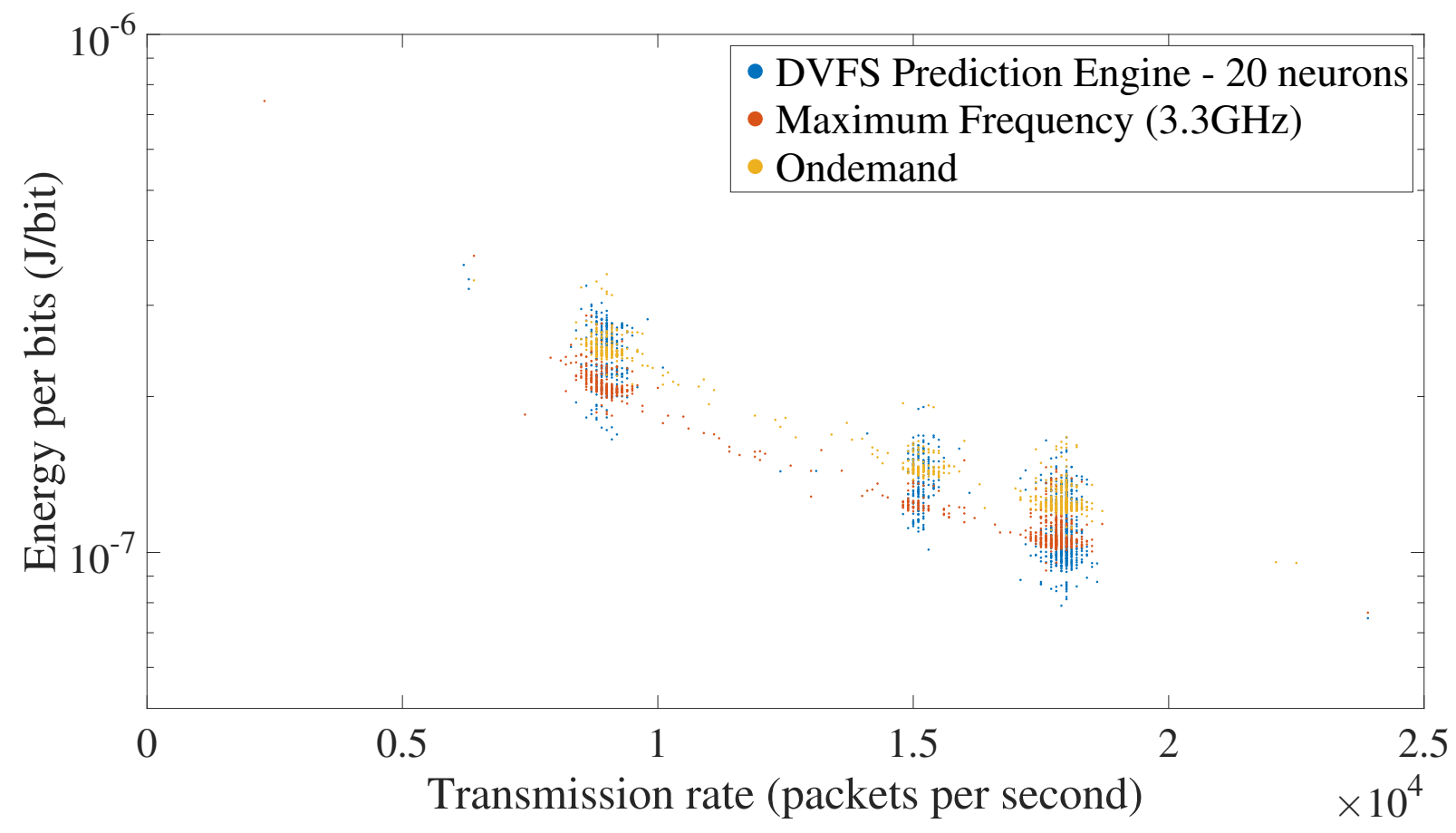

Source: Author 
Figure 28 presents the energy consumption as a function of the transmission rate for all scenarios analyzed.

Figure 28: Energy consumption per transmission rate for DVFS Prediction Engine with 20 and 200 neurons, maximum frequency, and ondemand governor.

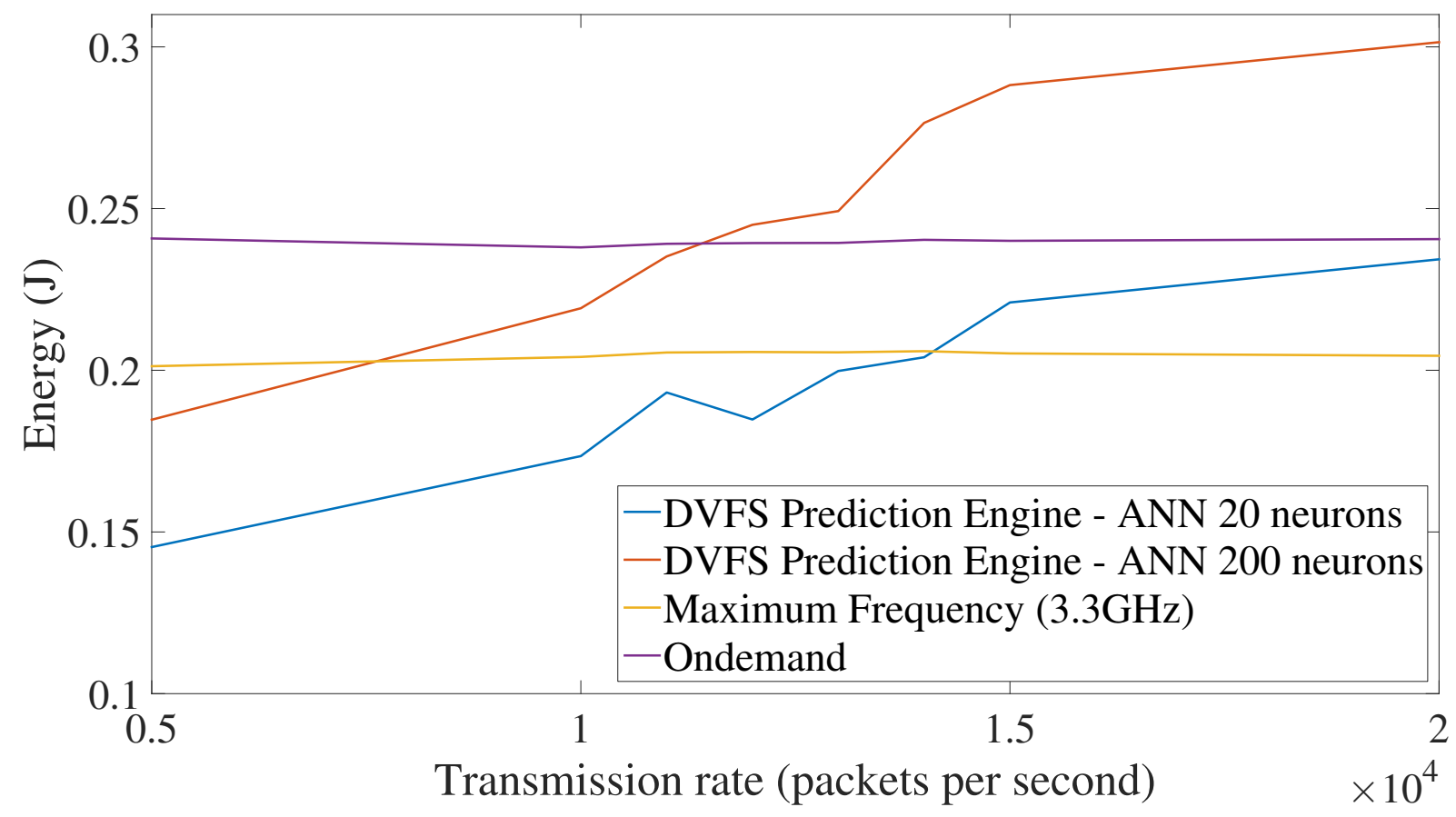

Source: Author

On maximum frequency and ondemand scenarios, the energy demand is rather steady, consuming approximately $0.2 \mathrm{~J}$ and $0.24 \mathrm{~J}$ at all transmission rates. Although ondemand governor scenario should dynamically select the processor frequency to manage the energy consumption and, thus, reduce it based on the CPU utilization, Figure 28 shows that it does not actually reduce the processors energy consumption.

Moreover, as expected based on previous results, the DVFS Prediction Engine with any number of neurons causes an increase in the energy consumption, as the transmission rate increases because of the use of higher frequencies and the computational overload generated by the ANN. Consequently, the DVFS Prediction Engine with 200 and 20 neurons curves exceed the maximum frequency results when the transmission rate is about 8,000 pps and 14,000 pps, respectively. Also, it surpasses the ondemand governor results when this value is approximately 11,000 pps and 20,000 pps for 200 and 20 neurons, respectively.

These results demonstrate that the method proposed in this work is able to provide better energy efficiency when compared to the industry best practice, i.e. using the maximum frequency, and to the in-kernel ondemand governor in some applications in 
which the data traffic did not cause great impact, as it occurs in small networks that process less than 14,000 pps. Moreover, because Data Centers do not have to process a great number of packets during all its execution time, DVFS Prediction Engine permits it to reduce its overall energy consumption in more than $12 \%$ when compared to maximum frequency, and in more than $27 \%$ in contrast to ondemand governor if the transmission rate is lower than 10,000 pps.

\subsection{CloudLab server}

To understand whether the solution is effective in other generations of Intel processors, the experiments were replicated in CloudLab (RICCI; EIDE, 2014), a testbed developed by University of Wisconsin, University of Utah and Clemson University that supports research in cloud computing. Since it is necessary to have full access to the servers when testing new cloud environments, the developers enable provision and control of the baremetal. Therefore, it is possible to perform kernel operations. Moreover, this section also presents a comparison between the two machine learning algorithms: Artificial Neural Networks (ANN) and Support Vector Machine (SVM).

The experiments were executed in a server with an Intel®Xeon®CPU E5-2630 v3 @2.40GHz with 128GB RAM. It had 32 CPUs when HyperThreading was enabled and 13 frequency steps that could be controlled by DVFS, from $1.2 \mathrm{GHz}$ to $2.4 \mathrm{GHz}$. As OS, it had Ubuntu 16.04 LTS installed.

\subsubsection{Training results}

Initially, a dataset to train the Machine Learning Module was built. As in the previous testbed, the maximum throughput that could be handled by sending traffic in the network with the TCPreplay host was estimated. Table 5 shows the transmission rate selected for each frequency in a way that the processing workload would surpass $90 \%$.

It is clear that when the processor operates at higher frequencies, it can handle a greater transmission rate, as the minimum frequency rate represents almost half of the maximum one. Furthermore, when compared to the previous testbed, it is possible to process more throughput: the maximum transmission rate that could be supported in the local testbed was 19,000 pps, while this server can handle up to 33,000 pps.

Figure 29 and Figure 30 presents, respectively, the energy consumption in $(\mathrm{J})$ and the number of cycles as a function of the packets received for all 13 frequencies. From these 
Table 5: Transmission rate selected per frequency for training with CloudLab server.

\begin{tabular}{ccc}
\hline$\#$ & Frequency $(\mathrm{GHz})$ & Throughput $(\mathrm{pps})$ \\
\hline 1 & 2.40 & 33,000 \\
2 & 2.30 & 31,500 \\
3 & 2.20 & 30,000 \\
4 & 2.10 & 27,500 \\
5 & 2.00 & 26,500 \\
6 & 1.90 & 25,000 \\
7 & 1.80 & 24,000 \\
8 & 1.70 & 23,500 \\
9 & 1.60 & 22,500 \\
10 & 1.50 & 22,000 \\
11 & 1.40 & 21,000 \\
12 & 1.30 & 18,000 \\
13 & 1.20 & 17,000 \\
\hline \multicolumn{3}{c}{ Source Author }
\end{tabular}

Source: Author.

images, it can be concluded that the frequency and transmission rate increase enables the execution of more cycles during the same amount of time and also leads to higher energy consumption. It also verified a variation in the transmission rate caused by the TCPreplay tool, that only guarantees the average rate.

Following the results in Section 5.2.1, the use of all 13 frequencies to train the Machine Learning Model could introduce errors in the prediction, because of the overlapping of frequencies both in terms of transmission rate and number of cycles executed, as Figure 29 and Figure 30 illustrate. Consequently, the Prediction Engine Module could misunderstand the best frequency to be chosen for a new dataset. Seven frequencies were therefore selected to train the Machine Learning Module as shown in Figure 31 and Figure 32.

The accuracy obtained in the offline validation step executed was evaluated with part of the data used for training both algorithms. Although ANN algorithm presented a reasonable precision when trained with the dataset composed by 13 frequencies - $90 \%$ with 50 neurons -, even higher accuracy was achieved when using only 7 of them $-96 \%$ with 20 neurons, as shown in Table 6. When training SVM algorithm, the accuracy obtained was lower for both datasets. Indeed, when SVM was trained with 7 frequencies, the accuracy was $85 \%$ when implemented with $\mathrm{C}$ and $\gamma$ equal to 10 and 1 , respectively, while the maximum accuracy when trained with 13 frequencies was $78 \%$, as presented in Table 7. The tables with all tested parameters can be found in Appendix A. 
Figure 29: Energy consumption per number of packets received for thirteen frequencies from $1.2 \mathrm{GHz}$ to $2.4 \mathrm{GHz}$.

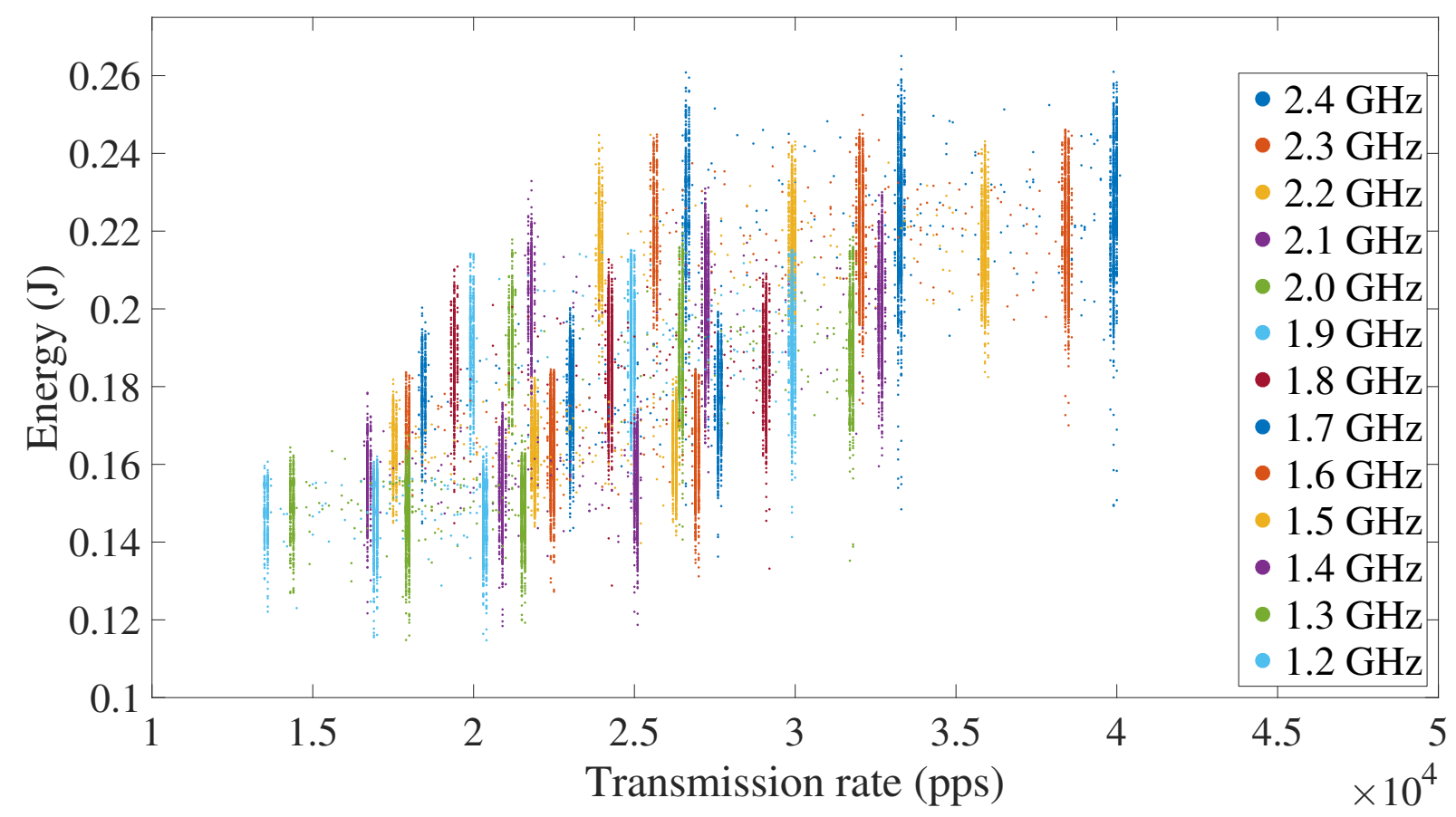

Source: Author

Figure 30: Number of cycles executed per number of packets received for thirteen frequencies from $1.2 \mathrm{GHz}$ to $2.4 \mathrm{GHz}$.

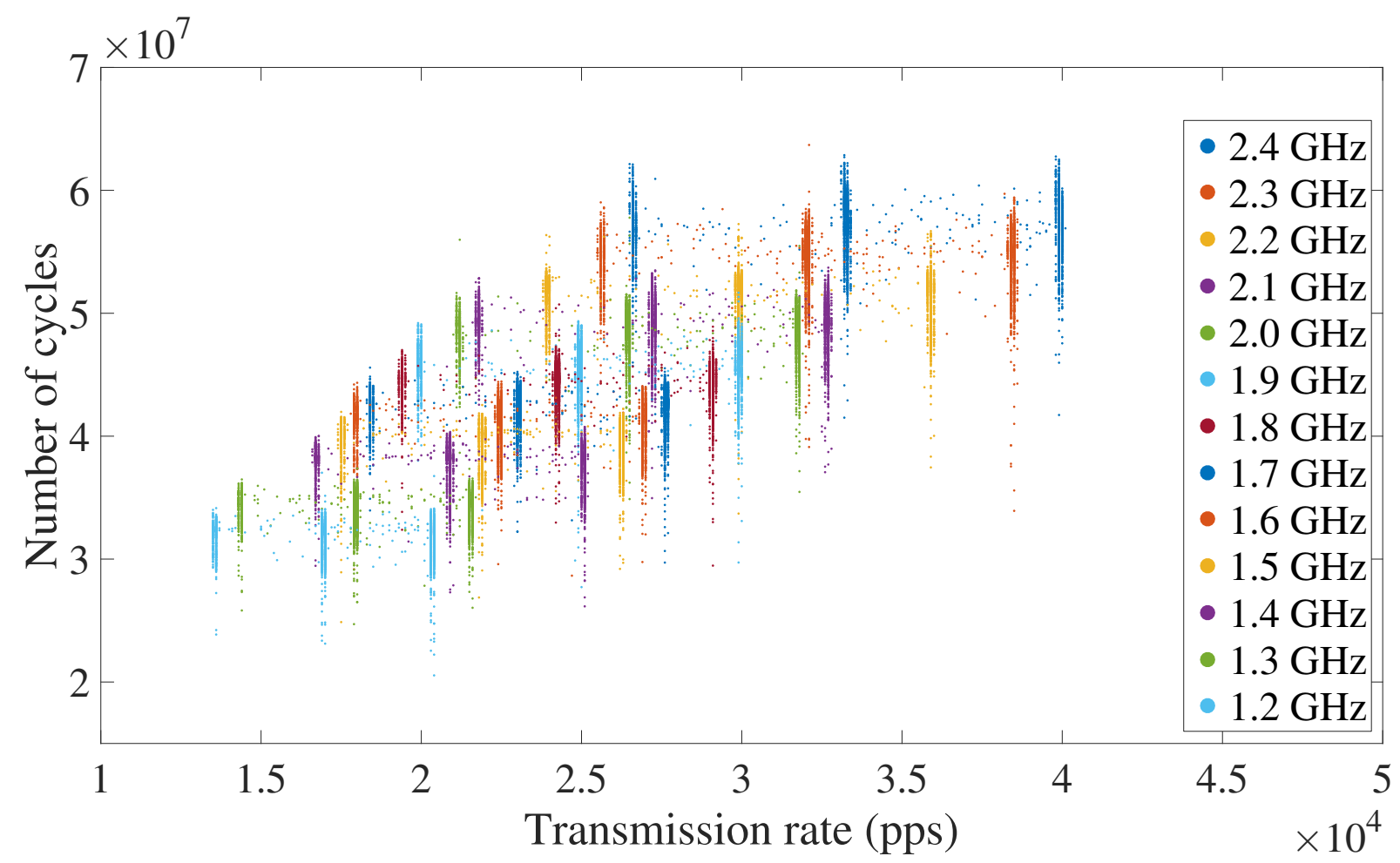

Source: Author 
Figure 31: Energy consumption per number of packets received for seven frequencies from $1.2 \mathrm{GHz}$ to $2.4 \mathrm{GHz}$.

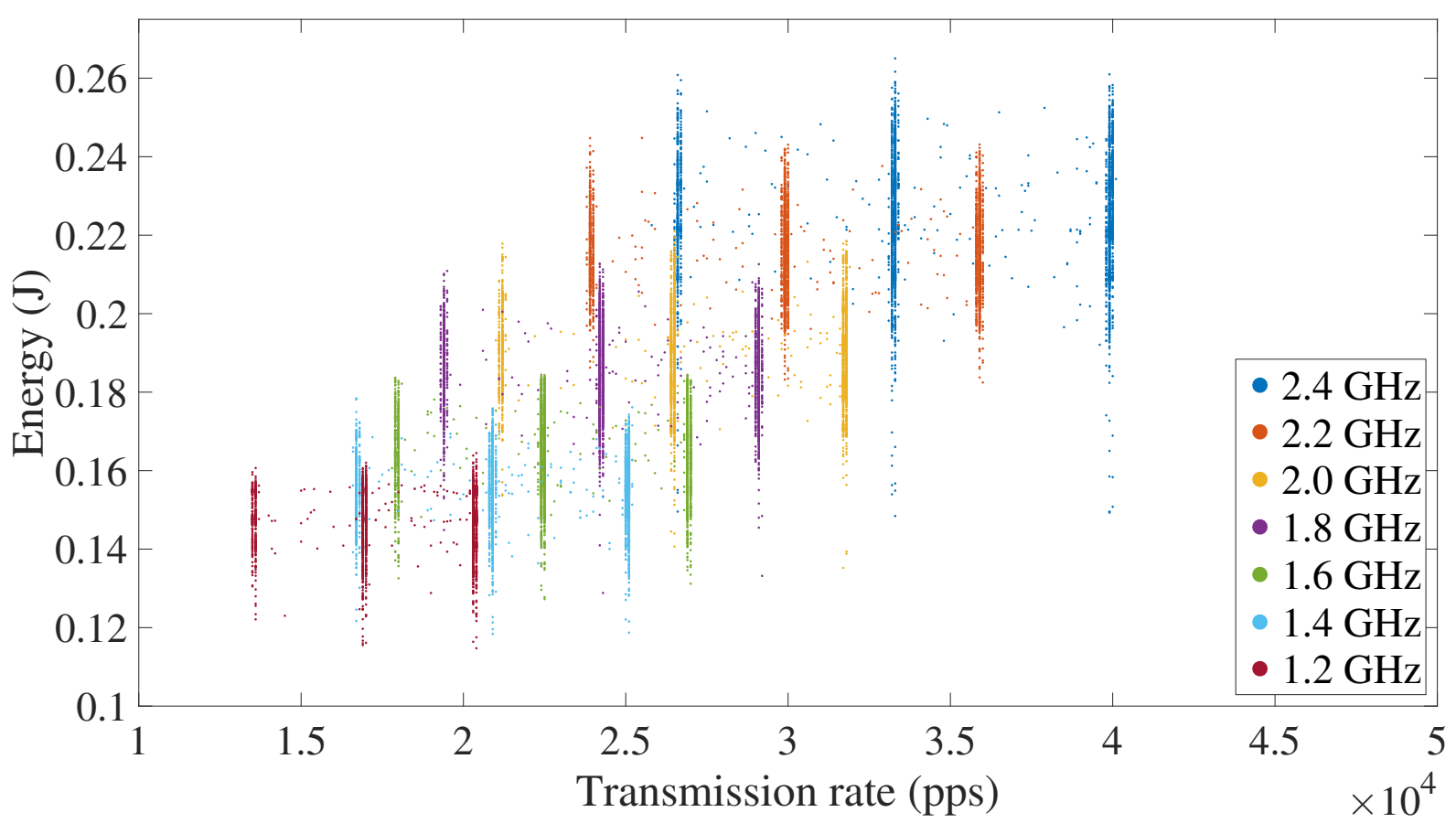

Source: Author

Figure 32: Number of cycles executed per number of packets received for seven frequencies from $1.2 \mathrm{GHz}$ to $2.4 \mathrm{GHz}$.

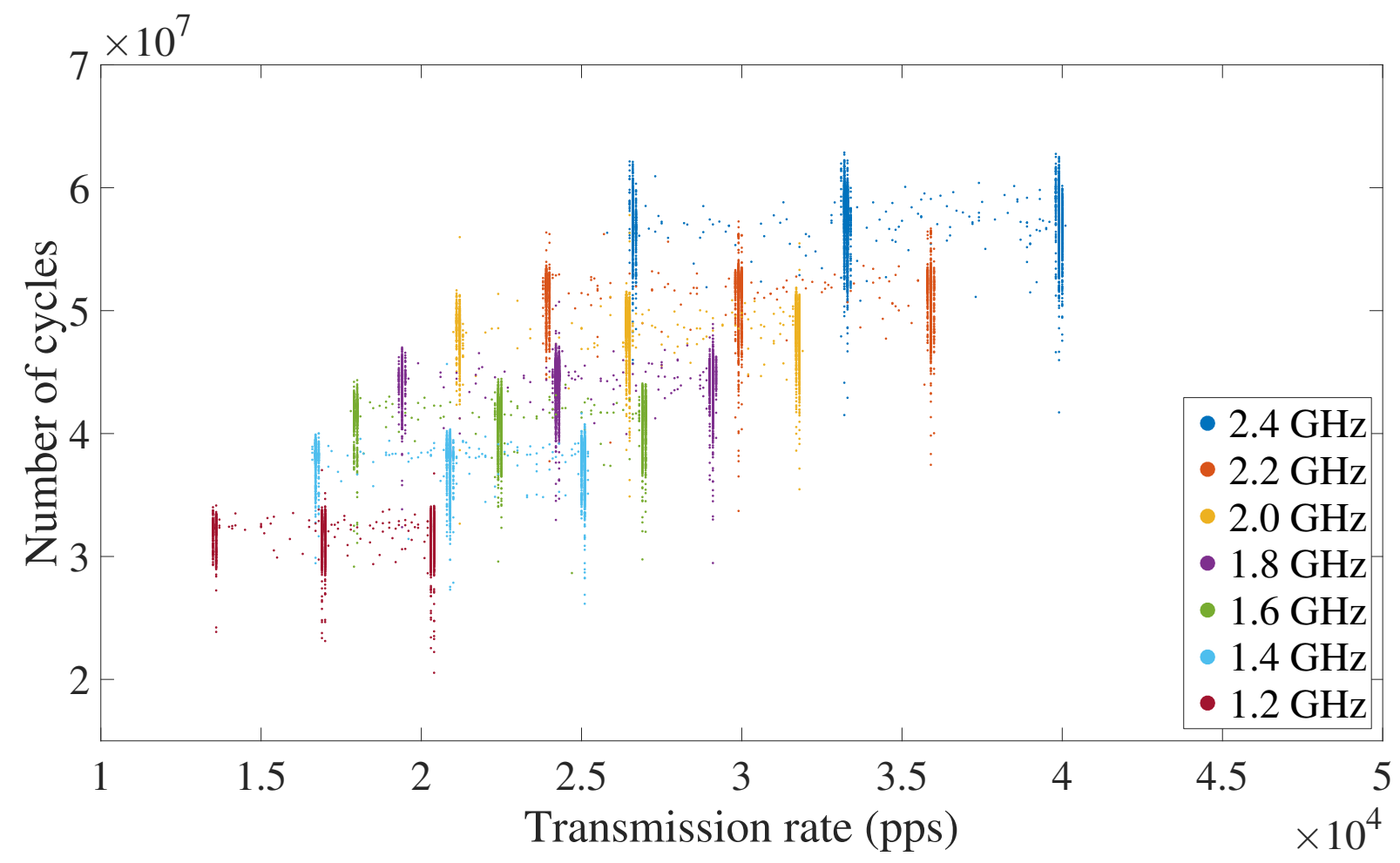

Source: Author 
Table 6: Highest accuracy values obtained for each set of ANN parameters in CloudLab server.

(a) Thirteen frequencies.

\begin{tabular}{cccc}
\hline Neurons & $\alpha$ & Batch & Accuracy \\
\hline 20 & 0.001 & 200 & $88.7 \%$ \\
20 & 0.001 & 400 & $88.4 \%$ \\
20 & 0.001 & 600 & $88.6 \%$ \\
20 & 0.01 & 200 & $88.9 \%$ \\
20 & 0.01 & 400 & $88.7 \%$ \\
20 & 0.01 & 600 & $88.6 \%$ \\
50 & 0.001 & 200 & $90 \%$ \\
50 & 0.001 & 400 & $88.4 \%$ \\
50 & 0.01 & 200 & $88.8 \%$ \\
50 & 0.01 & 400 & $88.7 \%$ \\
\hline
\end{tabular}

(b) Seven frequencies.

\begin{tabular}{cccc}
\hline Neurons & $\alpha$ & Batch size & Accuracy \\
\hline 20 & 0.001 & 200 & $95.7 \%$ \\
20 & 0.001 & 400 & $95.9 \%$ \\
20 & 0.001 & 600 & $96 \%$ \\
20 & 0.01 & 200 & $95.8 \%$ \\
20 & 0.01 & 400 & $95.7 \%$ \\
20 & 0.01 & 600 & $95.8 \%$ \\
20 & 0.1 & 600 & $94.8 \%$ \\
50 & 0.001 & 200 & $95 \%$ \\
50 & 0.001 & 600 & $95.2 \%$ \\
50 & 0.01 & 600 & $95 \%$ \\
\hline
\end{tabular}

Source: Author

Table 7: Highest accuracy values obtained for each set of SVM parameters for CloudLab server.

(a) Thirteen frequencies.

\begin{tabular}{ccc}
\hline $\mathrm{C}$ & $\gamma$ & Accuracy \\
\hline 1 & 1 & $72.2 \%$ \\
1 & 10 & $69.6 \%$ \\
10 & 1 & $77.7 \%$ \\
10 & 10 & $71.4 \%$ \\
100 & 0.1 & $67.6 \%$ \\
100 & 1 & $78.3 \%$ \\
100 & 10 & $71.3 \%$ \\
1000 & 0.1 & $76 \%$ \\
1000 & 1 & $78.5 \%$ \\
1000 & 10 & $71 \%$ \\
\hline
\end{tabular}

(b) Seven frequencies.

\begin{tabular}{ccc}
\hline $\mathrm{C}$ & $\gamma$ & Accuracy \\
\hline 1 & 1 & $82.6 \%$ \\
1 & 10 & $77.8 \%$ \\
10 & 1 & $85.4 \%$ \\
10 & 10 & $78.7 \%$ \\
100 & 0.1 & $78.9 \%$ \\
100 & 1 & $85.9 \%$ \\
100 & 10 & $78.5 \%$ \\
1000 & 0.1 & $86.4 \%$ \\
1000 & 1 & $85.1 \%$ \\
1000 & 10 & $78.3 \%$ \\
\hline
\end{tabular}

Source: Author

\subsubsection{Real-time results}

In the real-time scenario, the system extracts the same features and traffic statistics with which the Machine Learning Model was trained. The Prediction Engine Module receives in real-time an array of 53 features containing protocol information every $10 \mathrm{~ms}$. It analyzes them and uses the learning acquired in the Machine Learning Model during the training stage to estimate the best frequency. Depending on the frequency selected by the Prediction Engine Module, the DVFS Module adjusts immediately the frequency of the processors. 
This section describes the results in the real-time stage of the DVFS Prediction Engine executed with two different machine learning algorithms: ANN and SVM. Based on Section 5.3.1, ANN prediction engine with 20 neurons was implemented, which generated an accuracy of $96 \%$, and SVM prediction engine with $\mathrm{C}$ and $\gamma$ parameters equal to 10 and 1, respectively. These results were then compared to the ondemand governor and to the maximum frequency scenarios.

At first, the trace provided by Santanna et al. (2015) was introduced to obtain the real-time results. However, the SVM algorithm was not able to predict the frequency correctly with this trace, as its features are not similar from the one provided by Shiravi et al. (2012), which was used for training. A different part of the trace was therefore used for training to acquire the real-time results.

Figure 33 illustrates the energy consumption variation in a period of $1 \mathrm{~s}$ when the transmission rate is fixed to 15,000 pps in the TCPreplay host for all scenarios: DVFS Prediction Engine with ANN and with SVM, maximum frequency and ondemand governor. Evaluating the energy consumption of the DVFS Prediction Engine using ANN and SVM, they present very similar results, spending in average $0.19 \mathrm{~J}$ every $10 \mathrm{~ms}$. When compared to the maximum frequency, the DVFS Prediction Engine consumes in average $20 \%$ less. In addition, similarly to the previous testbed (Section 5.2.2), ondemand governor is the most power-demanding scenario, as it consumes in average almost $13 \%$ of the maximum frequency and more than $27 \%$ of both DVFS Prediction Engine implementations.

Figure 34 presents the energy efficiency - defined as the energy required to process every bit - for all scenarios. Since DVFS Prediction Engine using ANN and SVM has very similar energy consumption as observed in Figure 33, the scenario using ANN is hardly noticeable, because it is slightly hidden the SVM results.

The result expressed in the graph of Figure 34 is a consequence of Figure 33, because lower energy consumption to process the network traffic leads to higher energy efficiency. Indeed, the DVFS Prediction Engine scenarios present the lowest energy per bit as they require less energy to process the same amount of information from the network, which implies in higher energy efficiency.

Figure 35 and Figure 36 show the energy variation in time and the energy per bits, respectively, when the transmission rate is set to 33,000 pps for each scenario.

Although any important variation in the energy consumption of ondemand governor and maximum frequency scenarios was verified, the DVFS Prediction Engine with ANN and SVM showed a substantial increase in energy demand, reaching the maximum fre- 
Figure 33: Energy consumption in time for DVFS Prediction Engine with ANN and SVM, maximum frequency and ondemand governor at 15,000 pps.

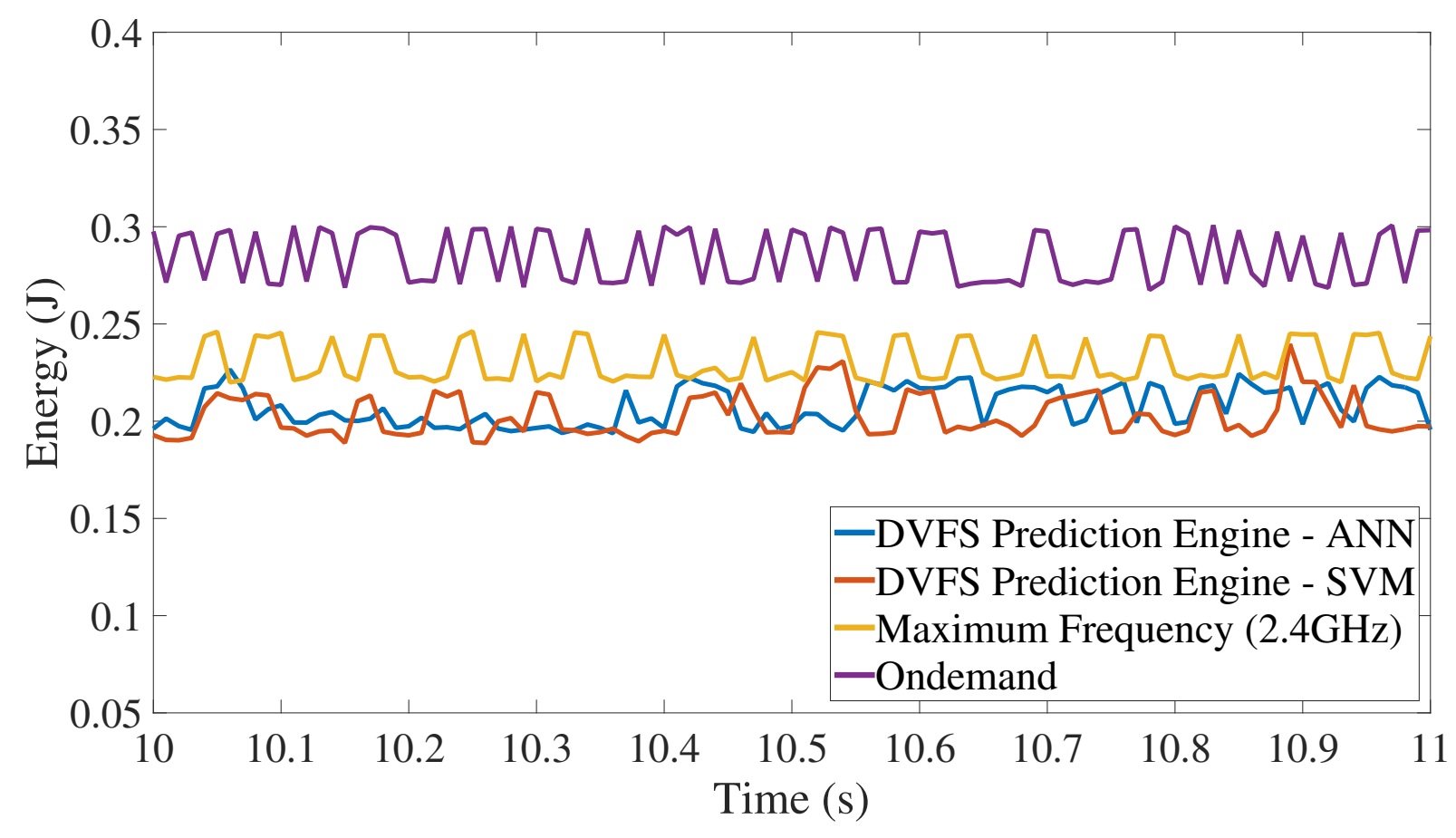

Source: Author

Figure 34: Energy efficiency per transmission rate for DVFS Prediction Engine with ANN and SVM, maximum frequency and ondemand governor at 15,000 pps.

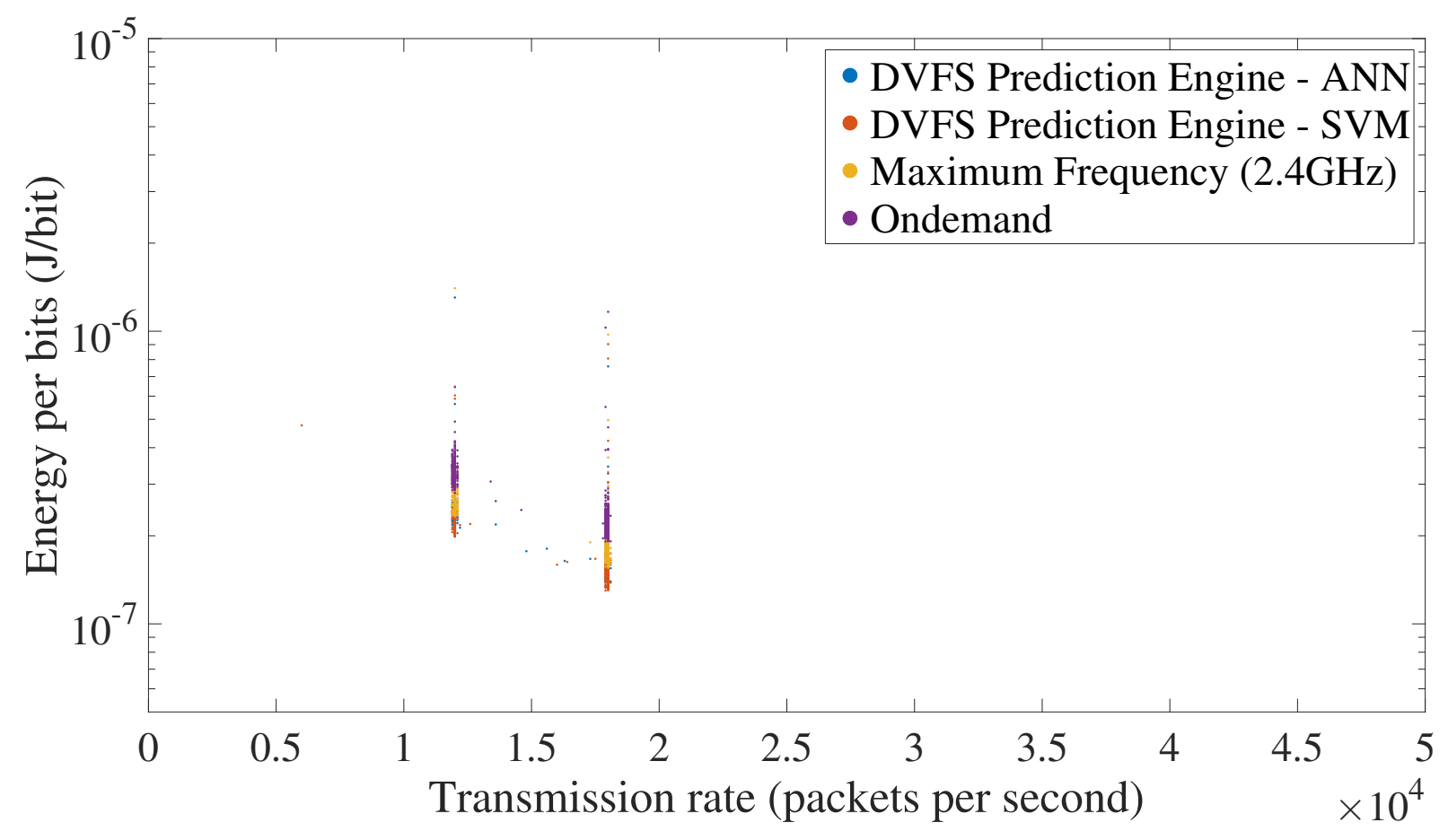

Source: Author 
Figure 35: Energy consumption in time for DVFS Prediction Engine with ANN and SVM, maximum frequency and ondemand governor at 33,000 pps.

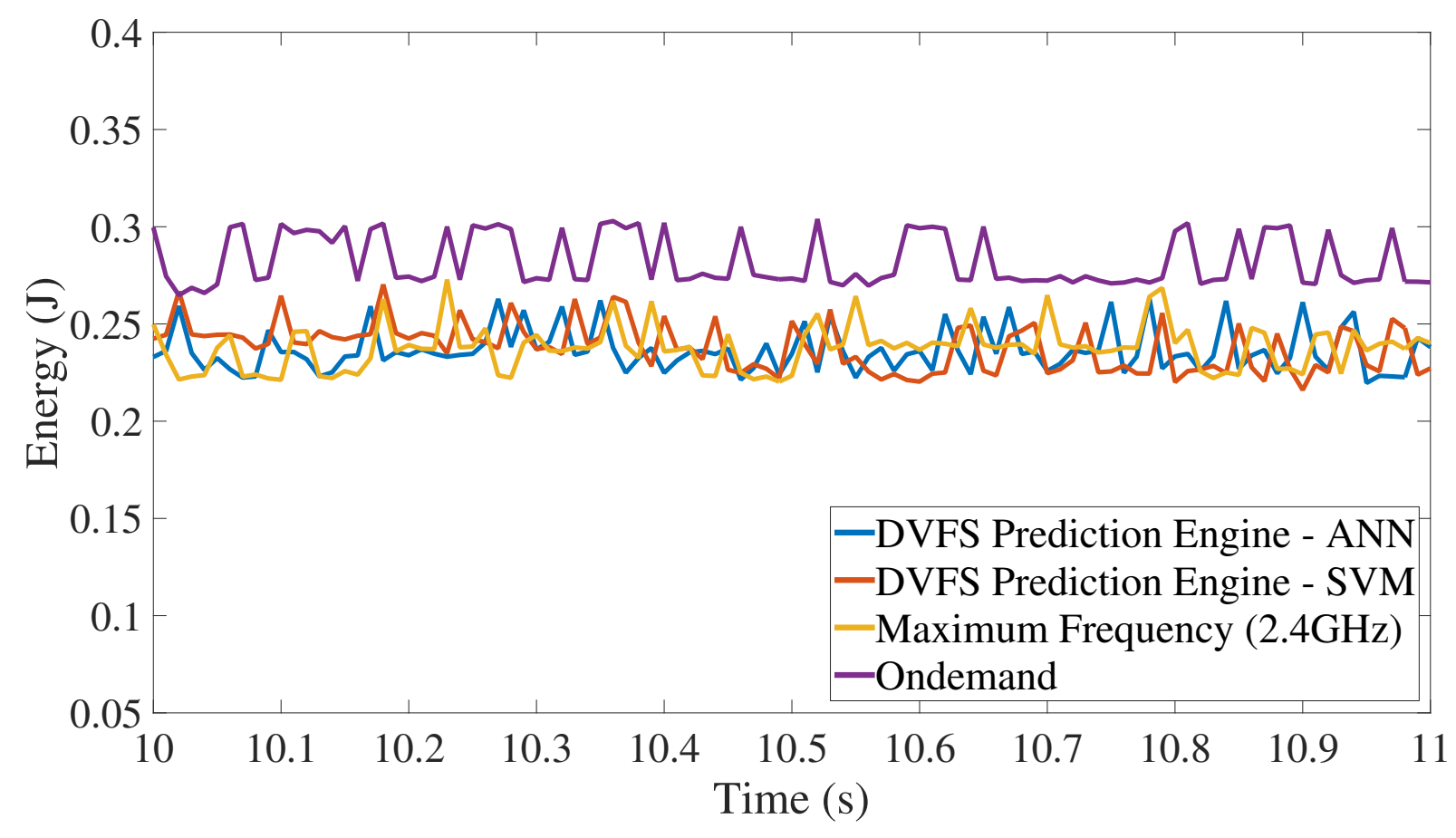

Source: Author

Figure 36: Energy efficiency per transmission rate for DVFS Prediction Engine with ANN and SVM, maximum frequency and ondemand governor at 33,000 pps.

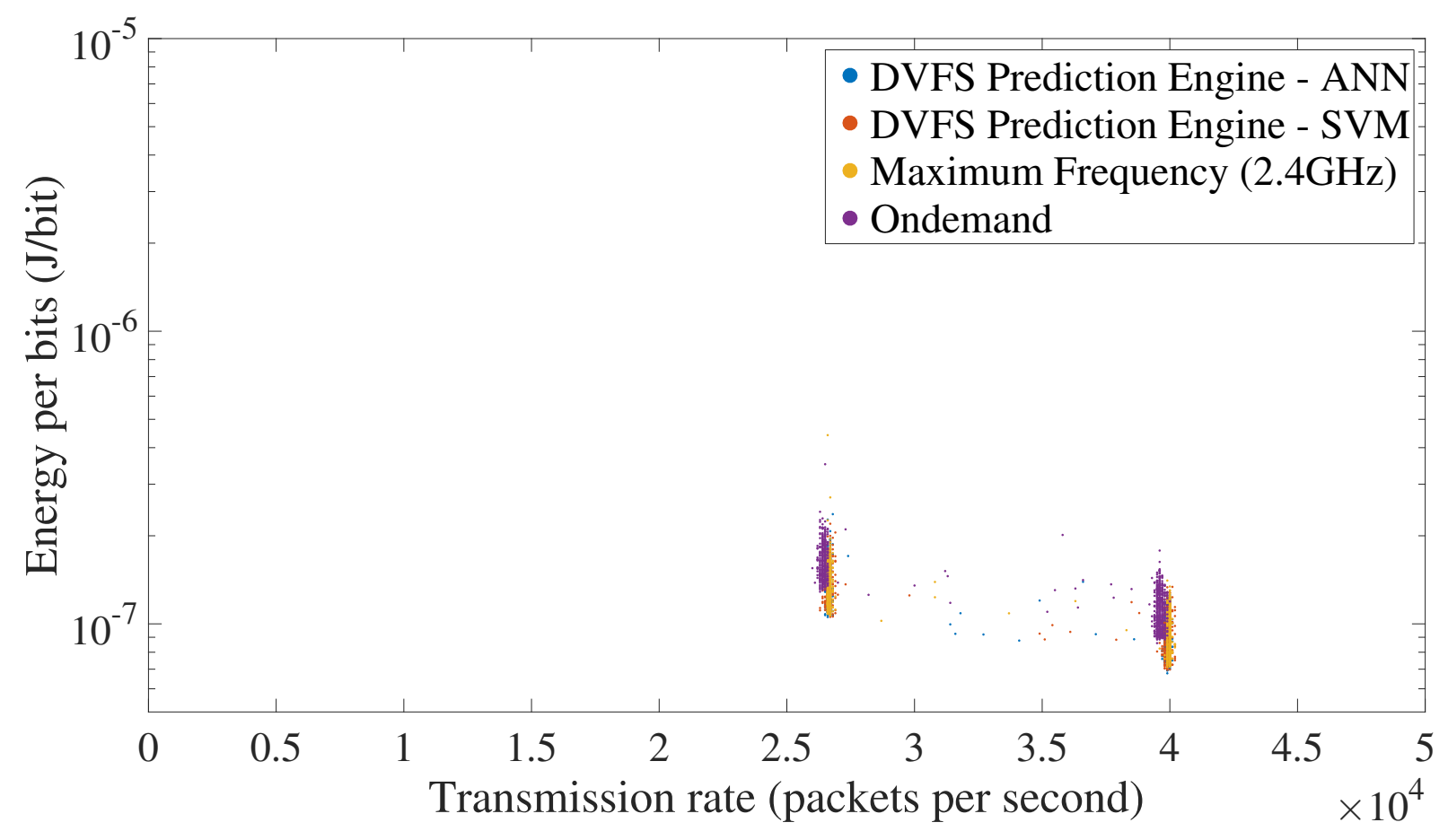

Source: Author 
quency curve. Similar to the local server Section 5.2.2, the prediction engine understands that higher frequencies must be selected in order to cope with the traffic arriving in the server. Furthermore, the use of a prediction engine generates extra processing. Hence, there is a higher consumption when the traffic rate is increased.

In order to understand this result better, measurements for each scenario at different transmission rates were performed. Figure 37 presents the results of these tests in a graph containing the average energy consumption as a function of the transmission rate for each scenario.

Both the maximum frequency and the ondemand governor scenarios are rather constant in average. As observed from previous measurements, the graph from Figure 37 also indicates that ondemand governor energy consumption is quite higher than the other scenarios even though it is in place Intel's solution to reduce energy according to the workload. It is clear that, in scenarios with NFV applications, the use of ondemand governor does not provide an optimal result.

The use of machine learning algorithms to predict the optimal operating frequency causes an increase in the energy consumption as the workload generated by the traffic processing is higher. Using SVM algorithm to implement the Prediction Engine Module first presents a reduction in the energy consumption, and, only when the transmission rate is set to $14,000 \mathrm{pps}$, it also starts increasing. This result comes from the fact that SVM selects mostly higher frequencies when the traffic rate is set between 5,000 and 14,000 pps; hence, it was not able to generalize correctly the training as ANN algorithm. DVFS Prediction Engine with ANN, on the other hand, has a fairly constantly ascending curve. Moreover, when the transmission rate is higher than 15,000 pps, the energy consumption of both algorithms is quite similar, being under the energy consumption of the maximum frequency in average until 33,000 pps at least.

Based on the results of this section, it can be concluded that the method proposed is able to reduce energy consumption compared to the maximum frequency (industry best practice) and to the ondemand governor in applications where the data traffic is under 33,000 pps. It is therefore possible to reduce the energy consumption in up to $18 \%$ with SVM and $21 \%$ with ANN when compared to the maximum frequency. Analyzing the DVFS Prediction Engine results in contrast to the ondemand governor, it is possible to save even more energy: $39 \%$ with SVM and 41\% with ANN. In addition, it was verified that the use of both machine learning algorithms present similar results when the average transmission rate is higher than 14,000 pps; nevertheless, SVM with the parameters used 
Figure 37: Energy consumption per transmission rate for DVFS Prediction Engine with ANN and SVM, maximum frequency and ondemand governor.

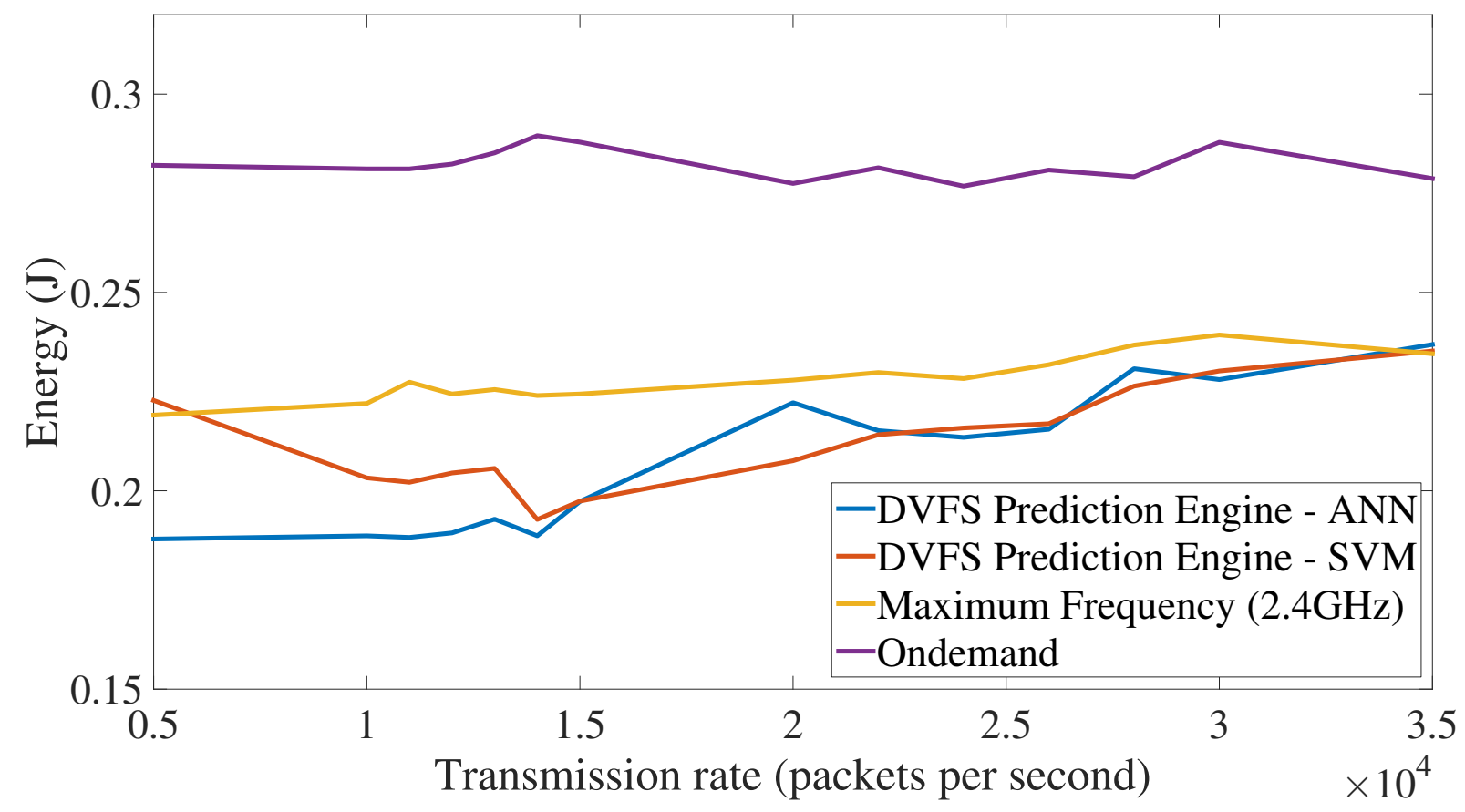

Source: Author

in this thesis was not able to efficiently predict the optimal frequency when the throughput was under 14,000 pps.

\subsection{Requirement Fulfillment}

Table 8 indicate whether the solution proposed in this thesis fulfill requirements described in Section 4.2 (represented by $\sqrt{ }$ ) or not (represented by $X$ ).

The solution proposed in this thesis is based on scaling the CPU frequency according to the packet characteristics. In order to achieve this goal, the DVFS framework was used and implemented using cpufreq library in Linux, satisfying FR1. The Network Module from the architecture presented in Section 4.3 sniffs the packets from the NIC and captures the packet features, such as the type of OSI protocols and the number of packets per protocol, to send it to the Prediction Engine Module that predicts the frequency that should be selected, fulfilling also FR2 FR3 and FR5. The features acquisition and frequency prediction are executed in a periodicity of $10 \mathrm{~ms}$ to ensure no packet loss because of a wrong prediction, which is in compliance with FR6.

Moreover, the DVFS Prediction Engine makes use of machine learning algorithms to 
Table 8: Requirements fulfillment

\begin{tabular}{l|l|l} 
Requirement & Requirement Name & $\begin{array}{c}\text { DVF Prediction } \\
\text { Engine }\end{array}$ \\
\hline FR1 & DVFS framework in the CPU & $\sqrt{ }$ \\
FR2 & Network traffic capturing & $\sqrt{ }$ \\
FR3 & Packet characteristics evaluation & $X$ \\
FR4 & Energy performance mode prediction & $\sqrt{ }$ \\
FR5 & Dynamic scaling & $\sqrt{ }$ \\
FR6 & Packet-processing performance & $X$ \\
FR7 & $\begin{array}{l}\text { Simple deployment in different processor } \\
\text { architectures }\end{array}$ & $\sqrt{ }$ \\
FR8 & Simple transition to other NFV applications \\
FR9 & Real environment testing & $\sqrt{ }$ \\
FR10 & & $\sqrt{ }$
\end{tabular}

Source: Author.

determine the best frequency according to the packet features. This solution makes it easy to translate the solution to different testbeds, as demonstrated by the experiments described in this section in which the system was implemented in servers with different Intel processors. Also, although this thesis does not demonstrate in the experiments, the machine learning simplifies the modification of VNF in the server, since the algorithm would only need to be trained once again. Hence, FR8 and FR9 were also fulfilled.

FR10 was also clearly satisfied as all the experiments presented in Section 5.2 and Section 5.3 were executed in real servers.

This table indicates that the only requirements that were not completely fulfilled are FR4 (CPU utilization evaluation) and FR7 (Packet-processing performance), because the solution only takes the performance and the CPU utilization into consideration when generating the training data set, and it is not evaluated during the real-time phase. 


\subsection{Chapter Final Remarks}

This chapter presents an evaluation of the main aspects of the DVFS Prediction Engine proposed in this thesis. It was divided in three main parts: the architecture implementation, the results in a server with a Sandy Bridge CPU and in a server with Haswell CPU.

In order to test the architecture proposed in Chapter 4, two Virtual Machines (VMs) were deployed: the TCPreplay host, responsible for generating the traffic, and the Snort host, responsible for processing the packets. Moreover, two different machine learning algorithms were implemented: Artificial Neural Networks (ANN) and Support Vector Machines (SVM). According to this testbed, the energy efficiency capabilities of the DVFS Prediction Engine was analyzed.

During the training process, the same traffic was replayed for different frequencies and the Network Module collected the packet features from the network, i.e. the types of OSI protocols and the number of packets per protocol. This dataset was used to train the Machine Learning Module. In the real-time phase, the traffic was sent at different transmission rates to evaluate the energy efficiency of the DVFS Prediction Engine. These results were compared to two different scenarios: maximum frequency and ondemand governor.

At first, the scenario was implemented in a local server containing Intel processors with Sandy Bridge microarchitecture, using only ANN algorithm (Section 5.2). In the training stage, it was verified that the accuracy increased substantially if a lower number of frequencies was used for training the ANN algorithm. Hence, eight out of fifteen frequencies were selected and it achieved $79 \%$ and $81 \%$ of precision when using 20 and 200 neurons, respectively, in the hidden layer. In the real-time phase, the method proposed in this thesis improved the energy efficiency when executing applications that do not require important data traffic, i.e. when the transmission rate is under 14,000 pps. Therefore, DVFS Prediction Engine reduced the processors energy consumption up to $12 \%$ and $27 \%$ when compared to maximum frequency and ondemand governor, respectively.

Next, the DVFS Prediction Engine results with two different machine learning algorithms (ANN and SVM) were evaluated in a newer server, which contains Intel processors with Haswell microarchitecture Section 5.3. During the training, it was clear that this server was more powerful than the previous one, as the supported throughput was higher. Moreover, ANN algorithm presented better accuracy, achieving 96\% when seven out of 
thirteen frequencies were used to train the Machine Learning Module. Using SVM algorithm, the calculated precision was $85 \%$, being considered also suitable for our application. The real-time analysis showed that both implementations of the DVFS Prediction Engine enable reducing the processor energy consumption compared to the ondemand governor and the maximum frequency. Indeed, it was possible to achieve up to $18 \%$ and $21 \%$ of reduction when using the DVFS Prediction Engine with SVM and ANN, respectively, compared to the maximum frequency. In addition, SVM with the parameters $\mathrm{C}$ as 10 and $\gamma$ as 1 is not able to generalize the training to lower throughput in this application, as it selected mostly the highest frequency when the transmission rate was set under 14,000 pps.

Based on the results presented in this chapter, it was concluded that the use of machine learning to scale the CPU frequency dynamically according to the traffic rate enables reducing the energy consumption in servers, with possible implementation making use of different Intel processor generations. Moreover, the DVFS Prediction Engine with ANN algorithm presented better results than SVM with the parameters tested, as it has an improved generalization capability in this scenario. 


\section{FINAL CONSIDERATIONS}

This chapter concludes the thesis by providing the final remarks in Section 6.1, which explain the main contribution of the thesis, the publications associated to this thesis in Section 6.2 and the work that could be done in the future in Section 6.3

\subsection{Main Contributions}

As the number of mobile devices and connected objects is increasing drastically in the past years, the need for improving network and processing performance becomes more and more important. The use of Network Function Virtualization (NFV) enables the scalability; however, it poses high processing performance constraints that result in great energy consumption. Based on this context, the DVFS Prediction Engine was proposed as a way of reducing the power consumption.

The solution proposed in this work presents a method for saving energy in the NFV domain. It is based on a Prediction Engine that analyzes the ongoing traffic and selects the optimal, i.e. the minimum frequency to which the processor can be set so that all packets can be correctly processed with greater energy efficiency.

In order to achieve these goals, this work was developed taking as basis different methods, listed as follows:

- Literature Review and Analysis: this step involved the elaboration of Chapter 2 and Chapter 3, which provides a theoretical basis for the Design and Development step.

Chapter 2: presented an explanation of the increase in energy consumption in servers and also an overview of a well-known solution to obtain energy efficiency in servers. The main contribution of this chapter is to position the reader about the technology that is used in this work. 
Chapter 3: provided an overview of current solutions to reduce energy consumption in data centers servers that employ NFV applications. The main contribution of this Chapter is to present more in deep the gap in the literature.

- Design and Development of the DVFS Prediction Engine: this step comprises the explanation of the technical solution that was proposed to meet the objective of this work and was described in Chapter 4 .

Chapter 4: introduced the DVFS Prediction Engine architecture and its main components detailing how they relate to each other. The architecture was based on five modules separated into user and kernel levels. The user level modules are responsible for training the machine learning algorithm and predicting the optimal frequency, while the kernel level modules need administrator action to capture the information to be used by the user level modules and modify the frequency. The main contribution of this Chapter is the architecture and technical description of the solution, explaining the machine learning algorithms used - Artificial Neural Networks (ANN) and Support Vector Machines (SVM) - and the modules capabilities. Moreover, this section also explores a method that is more accurate than current solutions, in which machine learning is employed to analyze packet features, such as the different types of protocols in the traffic.

- Evaluation: presents the experimental analyses of the DVFS Prediction engine described in Chapter 5.

Chapter 5: described the architecture implementation as well as the results obtained both during training and real-time phases. The results show that the solution developed is suboptimal when employed in Sandy Bridge processors in large networks, in which the virtual network function would need to analyze more than 14,000 packets per second. However, when considering small networks, it provides up to $27 \%$ and $12 \%$ of energy savings when compared to ondemand governor and the maximum frequency, respectively. Furthermore, considering a newer processor generation such as Haswell, the solution proposed in this thesis was able to save more than $19 \%$ when compared to the maximum frequency. The results also showed that the implementation of the Prediction Engine with Artificial Neural Networks was more effective than Support Vector Machines algorithm.

This work is the result of the author's collaboration in the project Energy Efficiency to Clouds (E2C) at the Laboratory of Sustainability of the Department of Computer Engineering and Digital Systems of Polytechnique School of Universidade de São Paulo 
(LASSU-PCS-EPUSP), which was developed in collaboration with Ericsson Telecomunicações S.A., Brazil, and Ericsson Research Sweden. Furthermore, this work was supported by CNPq (National Council for Scientific and Technological Development) Brazil (process $132234 / 2017-3)$.

\subsection{Publications}

As contributions directly related to this work, the following can be mentioned:

- 2018 - Paper/Published - Zorello, L. M. M., Vieira, M. G. T., Tejos, R. A. G., Rojas, M. A. T., Meirosu, C., Carvalho, T. C. M. B. Improving Energy Efficiency in NFV Clouds with Machine Learning. 2018 Workshop on Cloud Management and Operations, 2018 IEEE International Conference on Cloud Computing (CLOUD). July 2018.

- 2018 - Poster/Published - Zorello, L. M. M., Vieira, M. G. T., Tejos, R. A. G., Rojas, M. A. T., Meirosu, C., Carvalho, T. C. M. B. Improving Energy Efficiency in NFV Clouds with Machine Learning. 2018 IEEE World Conference on Services (IEEE SERVICES). July 2018.

In addition, during the Master, other publications were developed regarding Energy Efficiency and 5G:

- 2017 - Paper/Published - Zorello, L. M. M., Rojas, M. A. T., Coupechoux, M., Vaze, R., Carvalho, T. C. M. B. Performance Analysis of Online Matching Algorithms for D2D Communications. 2017 IEEE Latin-American Conference on Communications (LATINCOM). November 2017.

- 2017 - Paper/Published - Zorello, L. M. M., Carvalho, T. C. M. B. Comparative Analysis of Matching Algorithms for Green D2D Communication. VI Workshop de Pós Graduação - Engenharia de Computação (WPGEC). November 2017. Awarded with a honorable mention.

\subsection{Future work}

Despite providing a reduction in energy consumption in small networks, DVFS prediction engine requires improvements and further evaluation. Some potential future works worth being mentioned are: 
- Intel DPDK: in many scenarios, NFV employ technologies such as Intel Data Plane Development Kit (DPDK) that uses 100\% of the CPU cores allocated, regardless on whether traffic is present or not. An opportunity for saving more energy exists simply by adapting the frequency of these CPU cores to the incoming traffic rate. Thus, this scenario could be evaluated.

- Other types of virtual network functions: in this thesis, the DVFS Prediction Engine was evaluated using only Snort as a virtual network function. Executing different virtual network functions within the same server would allow to evaluate how the energy savings would follow different momentary workloads, and whether large differences in terms of frequency between the cores may have any negative effects on the overall performance.

- Other machine learning algorithms parameters: in spite of the fact that Artificial Neural Networks and Support Vector Machines with the parameters selected in this thesis provide reliable prediction and a considerable reduction in energy consumption, other parameters should be evaluated in order to compare their performance.

- Delay as another constraint: in this thesis, the CPU workload was evaluated based on the amount of traffic that the CPU can process at each frequency. Although this solution ensures that all packets will be handled at any frequency, the possible delay in packet processing that could be generated with the solution was not analyzed. It is therefore an opportunity of improving the system described in this thesis.

- Packet size as another constraint: as explained in Chapter 3 based on the work of Xu et al. (2016), the energy consumption in NFV applications is also impacted by the packet size. In the thesis, this information is not directly taken into consideration when determining the optimal operation mode. Hence, it could improve the results obtained in this thesis. 


\section{REFERENCES}

BOLLA, R. et al. Energy efficiency in the future internet: A survey of existing approaches and trends in energy-aware fixed network infrastructures. IEEE Communications Surveys and Tutorials, IEEE, v. 13, n. 2, p. 223-244, 2011. doi: 10.1109/SURV.2011.071410.00073.

BORKAR, S. et al. Platform 2015: Intel processor and platform evolution for the next decade. USA, 2005. Available from Internet: < https://www.cs.helsinki.fi/u/kerola/rio/ papers/borkar \_2015.pdf >.

BRODOWSKI, D. CPU frequency and voltage scaling code in the Linux(TM) kernel. Linux CPUFreq User Guide. 2017. Available from Internet: < https: //www.kernel.org/doc/Documentation/cpu-freq/user-guide.txt>. Accessed: April 15 2018.

CAO, L. et al. ENVI: Elastic resource flexing for network function virtualization. In: USENIX WORKSHOP ON HOT TOPICS IN CLOUD COMPUTING. 9. Proceedings... Santa Clara, CA: USENIX Association, 2017. Available from Internet: <https: //www.usenix.org/system/files/conference/hotcloud17/hotcloud17-paper-cao.pdf $>$.

COOK, G. How clean is your cloud? Greenpeace Inc., Amsterdam, The Netherlands, 2012.

COOK, G. et al. Clicking Clean: Who is Winning the Race to Build a Green Internet. Greenpeace Inc., Washington, DC, United States, 2017.

DESCHAMPS, J.-P.; VALDERRAMA, E.; TERES, L. Digital systems. In: Digital Systems - From Logic Gates to Processors. Barcelona, Spain: Springer International Publishing Switzerland, 2017. ISBN 978-3-319-41198-9. doi: 10.1007/978-3-319-41198-9.

DESROCHERS, S.; PARADIS, C.; WEAVER, V. M. A validation of dram rapl power measurements. In: INTERNATIONAL SYMPOSIUM ON MEMORY SYSTEMS. 2. Proceedings... Alexandria, VA, USA: ACM, 2016. p. 455-470. ISBN 978-1-4503-4305-3. doi: $10.1145 / 2989081.2989088$.

DOWECK, J. et al. Inside 6th-generation intel core: New microarchitecture codenamed skylake. IEEE Micro, v. 37, n. 2, p. 52-62, May 2017. ISSN 0272-1732. doi: 10.1109/MM.2017.38.

ERICSSON. Ericsson Mobility Report. Sweden, 2017.

ETSI. Network Functions Virtualization: An Introduction, Benefits, Enablers, Challenges \& Call for Action. [S.1.], 2012. Available from Internet: <https: //portal.etsi.org/nfv/nfv \_white $\backslash \_$paper.pdf $>$.

ETSI. Network Functions Virtualisation (NFV); Architectural Framework. France, 2013. 
GARCIA, L. M. Programming with libpcap - sniffing the network from our own application. Hakin9 Magazine, v. 3, n. 2, p. 38-46, 2008. ISSN 1733-7186.

GRANT, R. E. et al. Evaluating energy and power profiling techniques for hpc workloads. In: INTERNATIONAL GREEN AND SUSTAINABLE COMPUTING CONFERENCE. 8. Proceedings... Orlando, FL, USA: IEEE, 2017. p. 1-8. ISBN 978-1-5386-3470-7. doi: 10.1109/IGCC.2017.8323587.

GUIDO, S.; MüLLER, A. Introduction to machine learning with python - A Guide for Data Scientists. 1005 Gravenstein Highway North, Sebastopol, CA 95472: O'Reilly Media, 2016. ISBN 978-1-449-36941-5.

HACKENBERG, D. et al. An energy efficiency feature survey of the intel haswell processor. In: IEEE INTERNATIONAL PARALLEL AND DISTRIBUTED PROCESSING SYMPOSIUM WORKSHOPS. 29. Proceedings... Hyderabad, India: IEEE, 2015. v. 29, p. 896-904. ISBN 978-1-4673-7684-6. doi: 10.1109/IPDPSW.2015.70.

HAWILO, H. et al. Nfv: state of the art, challenges, and implementation in next generation mobile networks (vepc). IEEE Network, IEEE, v. 28, n. 6, p. 18-26, November 2014. ISSN 0890-8044. doi: 10.1109/MNET.2014.6963800.

HEARST, M. A. et al. Support vector machines. IEEE Intelligent Systems and their Applications, IEEE, v. 13, n. 4, p. 18-28, July 1998. ISSN 1094-7167. doi: $10.1109 / 5254.708428$.

INTEL. Intel 64 and IA-32 Architectures Software Developer's Manual - Volume 3B: System Programming Guide, Part 2. Santa Clara, CA, USA, 2018.

INTEL. Intel Xeon Processor Scalable Family: Datasheet, Volume One: Electrical. Santa Clara, CA, USA, 2018.

ISLAM, F. M. M. ul et al. Task aware hybrid DVFS for multi-core real-time systems using machine learning. Information Sciences, Elsevier, v. 433-434, p. 315-332, 2018. ISSN 0020-0255. doi: 10.1016/j.ins.2017.08.042.

KHAN, K. N. et al. Rapl in action: Experiences in using rapl for power measurements. ACM Transactions on Modeling and Performance Evaluation of Computing Systems, ACM, New York, NY, USA, v. 3, n. 2, p. 9:1-9:26, March 2018. ISSN 2376-3639. doi: $10.1145 / 3177754$.

KIM, K. H.; BUYYA, R.; KIM, J. Power aware scheduling of bag-of-tasks applications with deadline constraints on dvs-enabled clusters. In: IEEE INTERNATIONAL SYMPOSIUM ON CLUSTER COMPUTING AND THE GRID. 7. Proceedings... Rio de Janeiro, Brazil: IEEE, 2007. p. 541-548. ISBN 0-7695-2833-3. doi: 10.1109/CCGRID.2007.85.

KLIAZOVICH, D.; BOUVRY, P.; KHAN, S. U. Greencloud: A packet-level simulator of energy-aware cloud computing data centers. Journal of Supercomputing, Springer, v. 62, n. 2, p. 1263-1283, 2012. ISSN 0920-8542. doi: 10.1007/s11227-010-0504-1.

KOHONEN, T. An introduction to neural computing. Neural Networks, v. 1, n. 1, p. 3-16, 1988. ISSN 0893-6080. doi: 10.1016/0893-6080(88)90020-2. 
KRZYWDA, J. et al. Power-performance tradeoffs in data center servers: DVFS, cpu pinning, horizontal, and vertical scaling. Future Generation Computer Systems, Elsevier, v. 81, p. $114-128,2018$. ISSN 0167-739X. doi: 10.1016/j.future.2017.10.044.

KUANG, J.; BHUYAN, L.; KLEFSTA, R. Traffic-aware power optimization for network applications on multicore servers. In: DESIGN AUTOMATION CONFERENCE. 49. Proceedings... San Francisco, CA, USA: IEEE, 2012. p. 1006-1011. ISSN 0738-100X.

LEE, Y. C.; ZOMAYA, A. Y. Energy conscious scheduling for distributed computing systems under different operating conditions. IEEE Transactions on Parallel and Distributed Systems, IEEE, v. 22, n. 8, p. 1374-1381, August 2011. ISSN 1045-9219. doi: 10.1109/TPDS.2010.208.

LORENA, A. C.; CARVAlHO, A. C. P. L. F. D. Uma introdução as support vector machines. Revista de Informática Teórica e Aplicada, v. 14, n. 2, p. 43-67, 2007. ISSN 2175-2745. doi: 10.22456/2175-2745.5690.

MAROTTA, A.; KASSLER, A. A power efficient and robust virtual network functions placement problem. In: INTERNATIONAL TELETRAFFIC CONGRESS. 28. Proceedings... Würzburg, Germany: IEEE, 2016. v. 01, p. 331-339. doi: 10.1109/ITC-28.2016.151.

MELO, R.; PETRUCCI, V. Eficiência energética em navegação web usando DVFS. In: BRAZILIAN SYMPOSIUM ON COMPUTER NETWORKS AND DISTRIBUTED SYSTEMS. Proceedings... Campos do Jordão, Brazil: [s.n.], 2018. v. 36.

MIJUMBI, R. et al. Network function virtualization: State-of-the-art and research challenges. IEEE Communications Surveys Tutorials, v. 18, n. 1, p. 236-262, 2016. ISSN 1553-877X. doi: 10.1109/COMST.2015.2477041.

MOCHOCKI, B. et al. Network-aware dynamic voltage and frequency scaling. In: IEEE REAL TIME AND EMBEDDED TECHNOLOGY AND APPLICATIONS SYMPOSIUM. 13. Proceedings... Bellevue, WA, USA: IEEE, 2007. p. 215-224. ISSN 1545-3421. doi: 10.1109/RTAS.2007.20.

MOORE, G. E. Cramming more components onto integrated circuits, reprinted from electronics, volume 38, number 8, april 19, 1965, pp.114 ff. IEEE Solid-State Circuits Society Newsletter, v. 11, n. 3, p. 33-35, September 2006. ISSN 1098-4232. doi: 10.1109/N-SSC.2006.4785860.

NEHRU, E. I. et al. Neural load prediction technique for power optimization in cloud management system. In: IEEE CONFERENCE ON INFORMATION COMMUniCATION TECHNOLOGIES. 1. Proceedings... Thuckalay, India: IEEE, 2013. p. 541-544. ISBN 978-1-4673-5758-6. doi: 10.1109/CICT.2013.6558154.

NIELSEN, M. A. Neural Networks and Deep Learning. 1. ed. [S.l.]: Determination Press, 2015 .

PALLIPADI, V.; STARIKOVSKIY, A. The ondemand governor - past, present and future. In: LINUX SYMPOSIUM. 2. Proceedings... Ottawa, Canada: Linux Symposium, 2006. p. 215-230. 
PAN, T. et al. Towards power-aware network function virtualization on multi-core processors. In: IEEE CONFERENCE ON COMPUTER COMMUNICATIONS WORKSHOPS. 13. Proceedings... Honolulu, HI, USA: IEEE, 2018. ISBN 978-1-53865979-3. doi: 10.1109/INFCOMW.2018.8406858.

PEDREGOSA, F. et al. Scikit-learn: Machine learning in python. The Journal of Machine Learning Research, JMLR.org, v. 12, p. 2825-2830, November 2011. ISSN 1532-4435.

PELLEY, S. et al. Understanding and abstracting total data center power. In: WORKSHOP ON ENERGY EFFICIENT DESIGN. Proceedings... Austin, TX, USA: ACM, 2009. v. 11.

PETTEY, C. 5 Steps to Maximize Data Center Efficiency. 2016. Available from Internet: <https://www.gartner.com/smarterwithgartner/ 5-steps-to-maximize-data-center-efficiency/>. Accessed: April 42018.

RICCI, R.; EIDE, E. Introducing cloudlab: Scientific infrastructure for advancing cloud architectures and applications. ; login:: the magazine of USENIX $\&$ SAGE, USENIX Association, v. 39, n. 6, p. 36-38, 2014. ISSN ISSN 1044-6397.

RIEGER, F.; BOCKISCH, C. Survey of approaches for assessing software energy consumption. In: ACM SIGPLAN INTERNATIONAL WORKSHOP ON COMPREHENSION OF COMPLEX SYSTEMS. 2. Proceedings... New York, NY, USA: ACM, 2017. p. 19-24. ISBN 978-1-4503-5521-6. doi: 10.1145/3141842.3141846.

RIZVANDI, N. B.; TAHERI, J.; ZOMAYA, A. Y. Some observations on optimal frequency selection in DVFS-based energy consumption minimization. Journal of Parallel and Distributed Computing, Academic Press, Inc., v. 71, n. 8, p. 1154-1164, August 2011. ISSN 0743-7315. doi: 10.1016/j.jpdc.2011.01.004.

ROESCH, M. Snort - lightweight intrusion detection for networks. In: USENIX CONFERENCE ON SYSTEM ADMINISTRATION. 13. Proceedings... Berkeley, CA, USA: USENIX Association, 1999. p. 229-238. ISBN 978-1-931971-05-8.

ROSSI, F. D. et al. Green software development for multi-core architectures. In: IEEE SYMPOSIUM ON COMPUTERS AND COMMUNICATIONS. 19. Proceedings... Funchal, Portugal: IEEE, 2014. p. 1-6. ISSN 1530-1346. doi: 10.1109/ISCC.2014.6912565.

ROTEM, E. et al. Power-management architecture of the intel microarchitecture code-named sandy bridge. IEEE Micro, IEEE, v. 32, n. 2, p. 20-27, February 2012. ISSN 0272-1732. doi: 10.1109/MM.2012.12.

ROTEM, E. et al. H-earth: Heterogeneous multicore platform energy management. Computer, IEEE, v. 49, n. 10, p. 47-55, October 2016. ISSN 0018-9162. doi: 10.1109/MC.2016.309.

RUSU, S. et al. A $22 \mathrm{~nm}$ 15-core enterprise xeon x00ae; processor family. IEEE Journal of Solid-State Circuits, v. 50, n. 1, p. 35-48, Jan 2015. ISSN 0018-9200. doi: 10.1109/JSSC.2014.2368933. 
SAHA, S.; RAVINDRAN, B. An experimental evaluation of real-time DVFS scheduling algorithms. In: ANNUAL INTERNATIONAL SYSTEMS AND STORAGE CONFERENCE. 5. Proceedings... Haifa, Israel: ACM, 2012. p. 1-12. ISBN 978-1-4503-1448-0. doi: 10.1145/2367589.2367604.

SANTANNA, J. et al. Booters - an analysis of ddos-as-a-service attacks. In: IFIP/IEEE INTERNATIONAL SYMPOSIUM ON INTEGRATED NETWORK MANAGEMENT. 12. Proceedings... Ottawa, Canada: [s.n.], 2015. p. 243-251. doi: 10.1109/INM.2015.7140298.

SHIRAVI, A. et al. Toward developing a systematic approach to generate benchmark datasets for intrusion detection. Computers and Security, v. 31, n. 3, p. 357-374, 2012. ISSN 0167-4048. doi: 10.1016/j.cose.2011.12.012.

SUEUR, E. L.; HEISER, G. Dynamic voltage and frequency scaling: The laws of diminishing returns. In: INTERNATIONAL CONFERENCE ON POWER AWARE COMPUTING AND SYSTEMS. Proceedings... Vancouver, BC, Canada: USENIX Association, 2010. p. 1-8.

SULEIMAN, D.; IBRAHIM, M.; HAMARASH, I. Dynamic voltage frequency scaling (DVFS) for microprocessors power and energy reduction. In: INTERNATIONAL CONFERENCE ON ELECTRICAL AND ELECTRONICS ENGINEERING. 4. Proceedings... Orleans, Canada: International ASET Inc., 2005.

THEIS, T. N.; WONG, H. S. P. The end of moore's law: A new beginning for information technology. Computing in Science Engineering, v. 19, n. 2, p. 41-50, March 2017. ISSN 1521-9615. doi: 10.1109/MCSE.2017.29.

TURNER, A. Tcpreplay: Pcap editing and replay tool for NIX. 2014. Available from Internet: <http://tcpreplay.synfin.net/>. Accessed: April 182018.

VARASTEH, A.; GOUDARZI, M. Server consolidation techniques in virtualized data centers: A survey. IEEE Systems Journal, IEEE, v. 11, n. 2, p. 772-783, June 2017. ISSN 1932-8184. doi: 10.1109/JSYST.2015.2458273.

VARMA, A. et al. Power management in the intel xeon e5 v3. In: IEEE/ACM INTERNATIONAL SYMPOSIUM ON LOW POWER ELECTRONICS AND DESIGN. 15. Proceedings... Rome, Italy: IEEE, 2015. p. 371-376. doi: 10.1109/ISLPED.2015.7273542.

$\mathrm{XU}$, Z. et al. Demystifying the energy efficiency of network function virtualization. In: IEEE/ACM INTERNATIONAL SYMPOSIUM ON QUALITY OF SERVICE. 24. Proceedings... Beijing, China: IEEE, 2016. p. 1-10.

YU, Q.; ZNATI, T.; YANG, W. Energy-efficient, delay-aware packet scheduling in high-speed networks. In: IEEE INTERNATIONAL PERFORMANCE COMPUTING AND COMMUNICATIONS CONFERENCE. 34. Proceedings... Nanjing, China: IEEE, 2015. p. 1-8. doi: 10.1109/PCCC.2015.7410314.

ZHANG, H.; HOFFMAN, H. A quantitative evaluation of the rapl power control system. Feedback Computing, Citeseer, 2015. 
ZORELLO, L. M. M. et al. Improving energy efficiency in nfv clouds with machine learning. In: IEEE INTERNATIONAL CONFERENCE ON CLOUD COMPUTING WORKSHOP ON CLOUD MANAGEMENT AND OPERATIONS. 11. Proceedings... San Francisco, CA, USA: IEEE, 2018. 


\section{APPENDIX A - ACCURACY FOR LOCAL SERVER}

Appendix B presents the accuracy obtained for all parameters tested for Artificial Neural Networks (ANN) algorithm in local server. Table 9a and Table 9b provide the accuracy for ANN algorithm when thirteen and seven frequencies, respectively, are used for training

Table 9: Accuracy obtained for each set of ANN parameters in local server.

(a) Fifteen frequencies.

\begin{tabular}{cccc}
\hline Neurons & $\alpha$ & Batch & Accuracy \\
\hline 10 & 0.001 & 200 & $66.3 \%$ \\
10 & 0.001 & 400 & $64.9 \%$ \\
10 & 0.001 & 600 & $64 \%$ \\
10 & 0.01 & 200 & $65.3 \%$ \\
10 & 0.01 & 400 & $64.7 \%$ \\
10 & 0.01 & 600 & $64 \%$ \\
10 & 0.1 & 200 & $59.8 \%$ \\
10 & 0.1 & 400 & $60.9 \%$ \\
10 & 0.1 & 600 & $64.1 \%$ \\
20 & 0.001 & 200 & $69.1 \%$ \\
20 & 0.001 & 400 & $68.5 \%$ \\
20 & 0.001 & 600 & $67 \%$ \\
20 & 0.01 & 200 & $69 \%$ \\
20 & 0.01 & 400 & $68.5 \%$ \\
20 & 0.01 & 600 & $68.1 \%$ \\
20 & 0.1 & 200 & $66.4 \%$ \\
20 & 0.1 & 400 & $68.4 \%$ \\
20 & 0.1 & 600 & $69 \%$ \\
\hline
\end{tabular}

(b) Seven frequencies.

\begin{tabular}{cccc}
\hline Neurons & $\alpha$ & Batch size & Accuracy \\
\hline 10 & 0.001 & 200 & $66.3 \%$ \\
10 & 0.001 & 400 & $64.9 \%$ \\
10 & 0.001 & 600 & $64 \%$ \\
10 & 0.01 & 200 & $65.3 \%$ \\
10 & 0.01 & 400 & $64.7 \%$ \\
10 & 0.01 & 600 & $64 \%$ \\
10 & 0.1 & 200 & $59.8 \%$ \\
10 & 0.1 & 400 & $60.9 \%$ \\
10 & 0.1 & 600 & $64.1 \%$ \\
20 & 0.001 & 200 & $69.1 \%$ \\
20 & 0.001 & 400 & $68.5 \%$ \\
20 & 0.001 & 600 & $67 \%$ \\
20 & 0.01 & 200 & $69 \%$ \\
20 & 0.01 & 400 & $68.5 \%$ \\
20 & 0.01 & 600 & $68.1 \%$ \\
20 & 0.1 & 200 & $66.4 \%$ \\
20 & 0.1 & 400 & $68.4 \%$ \\
20 & 0.1 & 600 & $69 \%$ \\
\hline
\end{tabular}




\begin{tabular}{|c|c|c|c|c|c|c|c|}
\hline Neurons & $\alpha$ & Batch & Accuracy & Neurons & $\alpha$ & Batch size & Accuracy \\
\hline 50 & 0.001 & 200 & $71 \%$ & 50 & 0.001 & 200 & $71 \%$ \\
\hline 50 & 0.001 & 400 & $70.6 \%$ & 50 & 0.001 & 400 & $70.6 \%$ \\
\hline 50 & 0.001 & 600 & $70.6 \%$ & 50 & 0.001 & 600 & $70.6 \%$ \\
\hline 50 & 0.01 & 200 & $69.5 \%$ & 50 & 0.01 & 200 & $69.5 \%$ \\
\hline 50 & 0.01 & 400 & $69.3 \%$ & 50 & 0.01 & 400 & $69.3 \%$ \\
\hline 50 & 0.01 & 600 & $70.6 \%$ & 50 & 0.01 & 600 & $70.6 \%$ \\
\hline 50 & 0.1 & 200 & $68.4 \%$ & 50 & 0.1 & 200 & $68.4 \%$ \\
\hline 50 & 0.1 & 400 & $68.8 \%$ & 50 & 0.1 & 400 & $68.8 \%$ \\
\hline 50 & 0.1 & 600 & $69.4 \%$ & 50 & 0.1 & 600 & $69.4 \%$ \\
\hline 100 & 0.001 & 200 & $70.6 \%$ & 100 & 0.001 & 200 & $70.6 \%$ \\
\hline 100 & 0.001 & 400 & $69.5 \%$ & 100 & 0.001 & 400 & $69.5 \%$ \\
\hline 100 & 0.001 & 600 & $69.9 \%$ & 100 & 0.001 & 600 & $69.9 \%$ \\
\hline 100 & 0.01 & 200 & $71.1 \%$ & 100 & 0.01 & 200 & $71.1 \%$ \\
\hline 100 & 0.01 & 400 & $69.3 \%$ & 100 & 0.01 & 400 & $69.3 \%$ \\
\hline 100 & 0.01 & 600 & $69.7 \%$ & 100 & 0.01 & 600 & $69.7 \%$ \\
\hline 100 & 0.1 & 200 & $69 \%$ & 100 & 0.1 & 200 & $69 \%$ \\
\hline 100 & 0.1 & 400 & $69.1 \%$ & 100 & 0.1 & 400 & $69.1 \%$ \\
\hline 100 & 0.1 & 600 & $67.9 \%$ & 100 & 0.1 & 600 & $67.9 \%$ \\
\hline 200 & 0.001 & 200 & $70.8 \%$ & 200 & 0.001 & 200 & $70.8 \%$ \\
\hline 200 & 0.001 & 400 & $69.3 \%$ & 200 & 0.001 & 400 & $69.3 \%$ \\
\hline 200 & 0.001 & 600 & $71.8 \%$ & 200 & 0.001 & 600 & $71.8 \%$ \\
\hline 200 & 0.01 & 200 & $68.6 \%$ & 200 & 0.01 & 200 & $68.6 \%$ \\
\hline 200 & 0.01 & 400 & $68.9 \%$ & 200 & 0.01 & 400 & $68.9 \%$ \\
\hline 200 & 0.01 & 600 & $70.1 \%$ & 200 & 0.01 & 600 & $70.1 \%$ \\
\hline 200 & 0.1 & 200 & $66.7 \%$ & 200 & 0.1 & 200 & $66.7 \%$ \\
\hline 200 & 0.1 & 400 & $67.7 \%$ & 200 & 0.1 & 400 & $67.7 \%$ \\
\hline 200 & 0.1 & 600 & $67 \%$ & 200 & 0.1 & 600 & $67 \%$ \\
\hline 400 & 0.001 & 200 & $70.2 \%$ & 400 & 0.001 & 200 & $70.2 \%$ \\
\hline 400 & 0.001 & 400 & $68.4 \%$ & 400 & 0.001 & 400 & $68.4 \%$ \\
\hline 400 & 0.001 & 600 & $69.4 \%$ & 400 & 0.001 & 600 & $69.4 \%$ \\
\hline 400 & 0.01 & 200 & $69.8 \%$ & 400 & 0.01 & 200 & $69.8 \%$ \\
\hline 400 & 0.01 & 400 & $66.1 \%$ & 400 & 0.01 & 400 & $66.1 \%$ \\
\hline 400 & 0.01 & 600 & $69.3 \%$ & 400 & 0.01 & 600 & $69.3 \%$ \\
\hline 400 & 0.1 & 200 & $67.9 \%$ & 400 & 0.1 & 200 & $67.9 \%$ \\
\hline 400 & 0.1 & 400 & $65.3 \%$ & 400 & 0.1 & 400 & $65.3 \%$ \\
\hline 400 & 0.1 & 600 & $66.9 \%$ & 400 & 0.1 & 600 & $66.9 \%$ \\
\hline
\end{tabular}

Source: Author 


\section{APPENDIX B - ACCURACY FOR CLOUDLAB SERVER}

Appendix B presents the accuracy obtained for all parameters tested for Artificial Neural Networks (ANN) and Support Vector Machines (SVM) algorithms. Table 10a and Table 10b provide the accuracy for ANN algorithm when thirteen and seven frequencies, respectively, are used for training, while Table 11a and Table 11b present the accuracy obtained when training the SVM algorithm with thirteen and seven frequencies, respectively.

Table 10: Accuracy obtained for each set of ANN parameters in CloudLab server.

(a) Thirteen frequencies.

\begin{tabular}{cccc}
\hline Neurons & $\alpha$ & Batch & Accuracy \\
\hline 10 & 0.001 & 200 & $78.8 \%$ \\
10 & 0.001 & 400 & $77.6 \%$ \\
10 & 0.001 & 600 & $83.2 \%$ \\
10 & 0.01 & 200 & $76.4 \%$ \\
10 & 0.01 & 400 & $77 \%$ \\
10 & 0.01 & 600 & $77 \%$ \\
10 & 0.1 & 200 & $74.2 \%$ \\
10 & 0.1 & 400 & $75.4 \%$ \\
10 & 0.1 & 600 & $76.2 \%$ \\
20 & 0.001 & 200 & $88.7 \%$ \\
20 & 0.001 & 400 & $88.4 \%$ \\
20 & 0.001 & 600 & $88.6 \%$ \\
20 & 0.01 & 200 & $88.9 \%$ \\
20 & 0.01 & 400 & $88.7 \%$ \\
20 & 0.01 & 600 & $88.6 \%$ \\
20 & 0.1 & 200 & $84 \%$ \\
20 & 0.1 & 400 & $86 \%$ \\
20 & 0.1 & 600 & $84.8 \%$ \\
\hline
\end{tabular}

(b) Seven frequencies.

\begin{tabular}{cccc}
\hline Neurons & $\alpha$ & Batch size & Accuracy \\
\hline 10 & 0.001 & 200 & $87.5 \%$ \\
10 & 0.001 & 400 & $87.6 \%$ \\
10 & 0.001 & 600 & $88 \%$ \\
10 & 0.01 & 200 & $87.4 \%$ \\
10 & 0.01 & 400 & $87.5 \%$ \\
10 & 0.01 & 600 & $87.6 \%$ \\
10 & 0.1 & 200 & $83.1 \%$ \\
10 & 0.1 & 400 & $85 \%$ \\
10 & 0.1 & 600 & $85.6 \%$ \\
20 & 0.001 & 200 & $95.7 \%$ \\
20 & 0.001 & 400 & $95.9 \%$ \\
20 & 0.001 & 600 & $96 \%$ \\
20 & 0.01 & 200 & $95.8 \%$ \\
20 & 0.01 & 400 & $95.7 \%$ \\
20 & 0.01 & 600 & $95.8 \%$ \\
20 & 0.1 & 200 & $92.2 \%$ \\
20 & 0.1 & 400 & $92.7 \%$ \\
20 & 0.1 & 600 & $94.8 \%$ \\
\hline
\end{tabular}




\begin{tabular}{|c|c|c|c|c|c|c|c|}
\hline Neurons & $\alpha$ & Batch & Accuracy & Neurons & $\alpha$ & Batch size & Accuracy \\
\hline 50 & 0.001 & 200 & $90 \%$ & 50 & 0.001 & 200 & $95 \%$ \\
\hline 50 & 0.001 & 400 & $88.4 \%$ & 50 & 0.001 & 400 & $94.3 \%$ \\
\hline 50 & 0.001 & 600 & $87 \%$ & 50 & 0.001 & 600 & $95.2 \%$ \\
\hline 50 & 0.01 & 200 & $88.8 \%$ & 50 & 0.01 & 200 & $94.6 \%$ \\
\hline 50 & 0.01 & 400 & $88.7 \%$ & 50 & 0.01 & 400 & $93.4 \%$ \\
\hline 50 & 0.01 & 600 & $88.1 \%$ & 50 & 0.01 & 600 & $95 \%$ \\
\hline 50 & 0.1 & 200 & $83.4 \%$ & 50 & 0.1 & 200 & $92.1 \%$ \\
\hline 50 & 0.1 & 400 & $85.9 \%$ & 50 & 0.1 & 400 & $91.8 \%$ \\
\hline 50 & 0.1 & 600 & $85.8 \%$ & 50 & 0.1 & 600 & $94.5 \%$ \\
\hline 100 & 0.001 & 200 & $87.9 \%$ & 100 & 0.001 & 200 & $94.4 \%$ \\
\hline 100 & 0.001 & 400 & $87.7 \%$ & 100 & 0.001 & 400 & $93.2 \%$ \\
\hline 100 & 0.001 & 600 & $86.2 \%$ & 100 & 0.001 & 600 & $93.9 \%$ \\
\hline 100 & 0.01 & 200 & $87.9 \%$ & 100 & 0.01 & 200 & $94.3 \%$ \\
\hline 100 & 0.01 & 400 & $87.5 \%$ & 100 & 0.01 & 400 & $93 \%$ \\
\hline 100 & 0.01 & 600 & $86.3 \%$ & 100 & 0.01 & 600 & $93.4 \%$ \\
\hline 100 & 0.1 & 200 & $83.8 \%$ & 100 & 0.1 & 200 & $92.2 \%$ \\
\hline 100 & 0.1 & 400 & $85.6 \%$ & 100 & 0.1 & 400 & $88.8 \%$ \\
\hline 100 & 0.1 & 600 & $85.8 \%$ & 100 & 0.1 & 600 & $93.5 \%$ \\
\hline 200 & 0.001 & 200 & $87.5 \%$ & 200 & 0.001 & 200 & $94.2 \%$ \\
\hline 200 & 0.001 & 400 & $86.2 \%$ & 200 & 0.001 & 400 & $93.5 \%$ \\
\hline 200 & 0.001 & 600 & $86.6 \%$ & 200 & 0.001 & 600 & $94.3 \%$ \\
\hline 200 & 0.01 & 200 & $86.3 \%$ & 200 & 0.01 & 200 & $94.4 \%$ \\
\hline 200 & 0.01 & 400 & $86 \%$ & 200 & 0.01 & 400 & $92.9 \%$ \\
\hline 200 & 0.01 & 600 & $85.8 \%$ & 200 & 0.01 & 600 & $93.7 \%$ \\
\hline 200 & 0.1 & 200 & $83.7 \%$ & 200 & 0.1 & 200 & $91.9 \%$ \\
\hline 200 & 0.1 & 400 & $85 \%$ & 200 & 0.1 & 400 & $86.1 \%$ \\
\hline 200 & 0.1 & 600 & $85.3 \%$ & 200 & 0.1 & 600 & $91.6 \%$ \\
\hline 400 & 0.001 & 200 & $87.3 \%$ & 400 & 0.001 & 200 & $93.5 \%$ \\
\hline 400 & 0.001 & 400 & $85.3 \%$ & 400 & 0.001 & 400 & $88.1 \%$ \\
\hline 400 & 0.001 & 600 & $86.1 \%$ & 400 & 0.001 & 600 & $94.3 \%$ \\
\hline 400 & 0.01 & 200 & $86 \%$ & 400 & 0.01 & 200 & $93.2 \%$ \\
\hline 400 & 0.01 & 400 & $85.2 \%$ & 400 & 0.01 & 400 & $89.4 \%$ \\
\hline 400 & 0.01 & 600 & $85.6 \%$ & 400 & 0.01 & 600 & $92.3 \%$ \\
\hline 400 & 0.1 & 200 & $84 \%$ & 400 & 0.1 & 200 & $86.2 \%$ \\
\hline 400 & 0.1 & 400 & $85.1 \%$ & 400 & 0.1 & 400 & $83.7 \%$ \\
\hline 400 & 0.1 & 600 & $85.2 \%$ & 400 & 0.1 & 600 & $85.6 \%$ \\
\hline
\end{tabular}

Source: Author 
Table 11: Accuracy obtained for each set of SVM parameters for CloudLab server.

(a) Thirteen frequencies.

\begin{tabular}{ccc}
\hline $\mathrm{C}$ & $\gamma$ & Accuracy \\
\hline 0.001 & 0.1 & $17.4 \%$ \\
0.001 & 1 & $14.4 \%$ \\
0.001 & 10 & $8 \%$ \\
0.01 & 0.1 & $12.2 \%$ \\
0.01 & 1 & $35.1 \%$ \\
0.01 & 10 & $43.4 \%$ \\
0.1 & 0.1 & $18.9 \%$ \\
0.1 & 1 & $56.6 \%$ \\
0.1 & 10 & $58.9 \%$ \\
1 & 0.1 & $30.1 \%$ \\
1 & 1 & $72.2 \%$ \\
1 & 10 & $69.6 \%$ \\
10 & 0.1 & $52.8 \%$ \\
10 & 1 & $77.7 \%$ \\
10 & 10 & $71.4 \%$ \\
100 & 0.1 & $67.6 \%$ \\
100 & 1 & $78.3 \%$ \\
100 & 10 & $71.3 \%$ \\
1000 & 0.1 & $76 \%$ \\
1000 & 1 & $78.5 \%$ \\
1000 & 10 & $71 \%$ \\
\hline & &
\end{tabular}

(b) Seven frequencies.

\begin{tabular}{ccc}
\hline $\mathrm{C}$ & $\gamma$ & Accuracy \\
\hline 0.001 & 0.1 & $38.5 \%$ \\
0.001 & 1 & $31 \%$ \\
0.001 & 10 & $17 \%$ \\
0.01 & 0.1 & $27.2 \%$ \\
0.01 & 1 & $55.9 \%$ \\
0.01 & 10 & $54.6 \%$ \\
0.1 & 0.1 & $44 \%$ \\
0.1 & 1 & $71.1 \%$ \\
0.1 & 10 & $65.2 \%$ \\
1 & 0.1 & $57.5 \%$ \\
1 & 1 & $82.6 \%$ \\
1 & 10 & $77.8 \%$ \\
10 & 0.1 & $66.1 \%$ \\
10 & 1 & $85.4 \%$ \\
10 & 10 & $78.7 \%$ \\
100 & 0.1 & $78.9 \%$ \\
100 & 1 & $85.9 \%$ \\
100 & 10 & $78.5 \%$ \\
1000 & 0.1 & $86.4 \%$ \\
1000 & 1 & $85.1 \%$ \\
1000 & 10 & $78.3 \%$ \\
\hline
\end{tabular}

Source: Author 